\title{
FINAL REPORT ON GRAPHITE IRRADIATION TEST OG-1
}

by

\author{
R. J. PRICE and L. A. BEAVAN
}

Prepared under

Contract AT(04-3)-167

Project Agreement No. 17

for the

San Francisco Operations Office

U.S. Atomic Energy Commission

This document is

\section{PUDLCLY RELEASABLE}

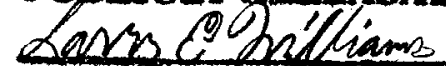

Autbotaing Ofricial

Date: $06 / 25 / 2907$

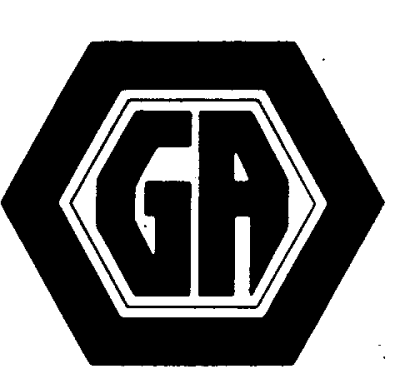

NOTICE

This report was prepared as an account of work This report was prepared as an account of work the United States nor the United States Atomic Energy Commission, nor any of their employees, nor any of Commission, nor any of their employees, nor any of their contractors, subcontractors, or their employees, makes any warranty, express or implied, or assumes any legal liability or responsibility for the accuracy, completeness or usefulness of any information, apparatus, product or process disclosed, or represents that its use would not infringe privately owned rights.

\section{GENERAL ATOMIC}




\section{DISCLAIMER}

This report was prepared as an account of work sponsored by an agency of the United States Government. Neither the United States Government nor any agency Thereof, nor any of their employees, makes any warranty, express or implied, or assumes any legal liability or responsibility for the accuracy, completeness, or usefulness of any information, apparatus, product, or process disclosed, or represents that its use would not infringe privately owned rights. Reference herein to any specific commercial product, process, or service by trade name, trademark, manufacturer, or otherwise does not necessarily constitute or imply its endorsement, recommendation, or favoring by the United States Government or any agency thereof. The views and opinions of authors expressed herein do not necessarily state or reflect those of the United States Government or any agency thereof. 


\section{DISCLAIMER}

Portions of this document may be illegible in electronic image products. Images are produced from the best available original document. 
SUMMARY

This report details the results of dimensional measurements and physical property tests on graphite samples irradiated in capsule OG-1. Samples of $\mathrm{H}-327$ (needle-coke graphite), $\mathrm{H}-429$ and $\mathrm{H}-451$ (near-isotropic petroleum-coke graphites), $P_{3}$ JHAN (pitch-coke graphite), and other miscellaneous graphites were irradiated at temperatures from $600^{\circ}$ to $1400^{\circ} \mathrm{C}$ to fluences from 1.6 to $3.7 \times 10^{21} \mathrm{n} / \mathrm{cm}^{2}$ ( $E>0.18 \mathrm{MeV}$ ).

Irradiation-induced shrinkages were smaller and more isotropic in the H-429 and H-451 graphites than in either the needle-coke or pitch-coke graphites irradiated under similar conditions.

Irradiation-induced changes in the thermal expansivity of $\mathrm{H}-327$ graphite were insignificant. Irradiation caused a small increase in the thermal expansivity of $\mathrm{H}-429$ and $\mathrm{H}-451$ graphites at irradiation temperatures below about $900^{\circ} \mathrm{C}$ and a slight decrease at higher temperatures.

Irradiation reduced both the thermal conductivity of $\mathrm{H}-327$ and $\mathrm{H}-451$ graphites and its temperature dependence. After irradiation at $600^{\circ}$ and $900^{\circ} \mathrm{C}$, the radial conductivity of $\mathrm{H}-451$ graphite was about $50 \%$ higher than the radial conductivity of $\mathrm{H}-327$ graphite and about equal to the axial conductivity of $\mathrm{H}-327$ graphite.

Irradiation increased both the strength and Young's modulus of H-327 and H-451 graphites, with a greater increase at lower irradiation temperatures than at higher irradiation temperatures. Percentage increases in strength fell in the range $30 \%$ to $60 \%$, while increases in Young's modulus ranged from $60 \%$ to $130 \%$. 


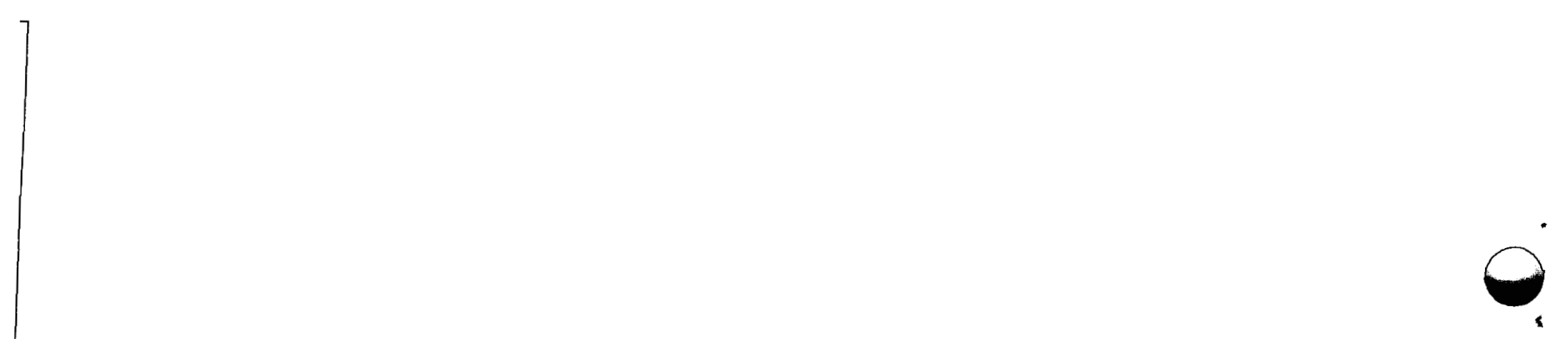


CONTENTS

SUMMARY . . . . . . . . . . . . . . . . . . . . . . . iii

1. INTRODUCTION . . . . . . . . . . . . . . . . . . . . . . 1

2. TEST DESCRIPTION AND OBJECTIVES . . . . . . . . . . . . . . . . 2

3. MATERIALS INVESTIGATED . . . . . . . . . . . . . . . . . 4

4. CAPSULE OPERATING COINDITIONS . . . . . . . . . . . . . . . 7

4.1. Description of Capsule . . . . . . . . . . . . . . 7

4.2. Operating Temperatures . . . . . . . . . . . . . 7

4.3. Neutron Fluences . . . . . . . . . . . . . . 10

5. EXPERIMENTAL RESULTS - DIMENSTONAL CHANGES . . . . . . . . . . 13

5.1. Irradiation-Induced Dimensional Change in Near-Isotropic

Graphites . . . . . . . . . . . . . . . 13

5.2. Dimensional Change in Needle-Coke Graphites . . . . . . . 40

5.3. Miscellaneous Graphites . . . . . . . . . . . . . 40

5.4. Comparison . . . . . . . . . . . . . . . . . 59

5.5. Carbon Insulating Materials .. . . . . . . . . . . . 59

6. EXPERIMENTAL RESULTS - THERMAL PROPERTIES . . . . . . . . . . 62

6.1. Thermal Expansivity . . . . . . . . . . . . . . . 62

6.2. Thermal Conductivity . . . . . . . . . . . . . . 69

7. EXPERIMENTAL RESULTS - TENSILE PROPERTIES . . . . . . . . . . . 78

8. IMPACT OF TEST DATA ON CURRENT DESIGN-BASIS CURVES . . . . . . . 88

8.1. Dimensional Changes . . . . . . . . . . . . . . 88

8.2. Thermal Expansivity . . . . . . . . . . . . . . 88

8.3. Thermal Conductivity . . . . . . . . . . . . . 89

8.4. Mechanical Properties . . . . . . . . . . . . . 89

9. CONCLUSIONS . . . . . . . . . . . . . . . . . . 90

ACKNOWLEDGMENTS . . . . . . . . . . . . . . . . . . . . . . 91

REFERENCES. . . . . . . . . . . . . . . . . . . . 92 


\section{FIGURES}

1. Fluence as a function of axial position in capsule OG-1 . . . .

2a. Dimensional changes of near-isotropic graphites $\mathrm{H}-451 / \mathrm{H}-429$ in the parallel direction for irradiation temperatures of $550^{\circ}$

and $750^{\circ} \mathrm{C}$. . . . . . . . . . . . . . . . .

2b. Dimensional changes of near-isotropic graphites $\mathrm{H}-451 / \mathrm{H}-429$ in the parallel direction for irradiation temperatures of $650^{\circ}$ and $850^{\circ} \mathrm{C}$. . . . . . . . . . . . . . . . .

2c. Dimensional changes of near-isotropic graphites $\mathrm{H}-451 / \mathrm{H}-429$ in the parallel direction for irradiation temperatures of $950^{\circ}$ and $1150^{\circ} \mathrm{C}$. . . . . . . . . . . . . . . . .

2d. Dimensional changes of near-isotropic graphites $\mathrm{H}-451 / \mathrm{H}-429$ in the parallel direction for irradiation temperatures of $1050^{\circ}$, $1250^{\circ}$, and $1350^{\circ} \mathrm{C}$. . . . . . . . . . . . . .

3a. Dimensional changes of near-isotropic graphites $\mathrm{H}-451 / \mathrm{H}-429$ in the perpendicular direction for irradiation temperatures of $550^{\circ}$ and $750^{\circ} \mathrm{C}$

3b. Dimensional changes of near-isotropic graphites $H-451 / H-429$ in the perpendicular direction for irradiation temperatures of $650^{\circ}$ and $850^{\circ} \mathrm{C}$. . . . . . . . . . . . . . . .

3c. Dimensional changes of near-isotropic graphites $\mathrm{H}-451 / \mathrm{H}-429$ in the perpendicular direction for irradiation temperatures of $950^{\circ}$ and $1150^{\circ} \mathrm{C}$. . . . . . . . . . . . . . . .

3d. Dimensional changes of near-isotropic graphites $\mathrm{H}-451 / \mathrm{H}-429$ in the perpendicular direction for irradiation temperatures of $1050^{\circ}, 1250^{\circ}$, and $1350^{\circ} \mathrm{C}$. . . . . . . . . . .

4. Irradiation-induced dimensional changes in near-isotropic graphite; parallel (axial) orientation . . . . . . . . . .

5. Irradiation-induced dimensional changes in near-isotropic graphite; perpendicular (radial) orientation . . . . . . . .

6. Thermal expansivity of $\mathrm{H}-327$ graphite as a function of fast neutron fluence. . . . . . . . . . . . . . . . .

7a. Thermal expansivity of $\mathrm{H}-451$ and $\mathrm{H}-429$ graphites irradiated at $640^{\circ}$ to $1060^{\circ} \mathrm{C}$ as a function of fast neutron fluence . . . . .

7b. Thermal expansivity of $\mathrm{H}-451$ and $\mathrm{H}-429$ graphites irradiated at $1050^{\circ}$ to $1500^{\circ} \mathrm{C}$ as a function of fast neutron fluence . . .

8. Thermal conductivity at room temperature of H-327 graphite as a function of fast neutron fluence . . . . . . . . . . . .

9. Thermal conductivity at irradiation temperature of $\mathrm{H}-327$ graphite as a function of fast neutron fluence . . . . . .

10. Thermal conductivity at room temperature of $\mathrm{H}-451$ graphite as a function of fast neutron fluence 
11. Thermal conductivity at irradiation temperature of $\mathrm{H}-451$ graphite as a function of fast neutron fluence . . . . . . . 75

12. Thermal conductivity of unirradiated and irradiated $\mathrm{H}-327$ and H-451 graphites (perpendicular orientation) as a function of measurement temperature . . . . . . . . . . . 76

13. Typical stress-strain curves of unirradiated and irradiated H-451 graphite. . . . . . . . . . . . . . . . . . .

14a. Ultimate tensile strength and Young's modulus of $\mathrm{H}-327$ graphite irradiated at $640^{\circ}$ to $900^{\circ} \mathrm{C}$ as a function of fast neutron fluence . . . . . . . . . . . . . . . . . . . . . .

14b. U1timate tensile strength and Young's modulus of H-327 graphite irradiated at $960^{\circ}$ to $1100^{\circ} \mathrm{C}$ as a function of fast neutron

fluence .......................

15. U1timate tensile strength and Young's modulus of H-451 graphite as a function of fast neutron fluence . . . . . . . . . . . .

TABLES

1. Graphites and miscellaneous materials in $O G-1$. . . . . . . . 5

2. Average temperature estimates for $O G-1$ experiment . . . . . . . 8

3. Mean temperatures and fluences in capsule OG-1 . . . . . . . . . 11

4a. Dimensional change in $\mathrm{H}-451$ and $\mathrm{H}-429$ graphites (parallel orientation) . . . . . . . . . . . . . . . 22

4b. Dimensional change in $\mathrm{H}-451$ and $\mathrm{H}-429$ graphites (perpendicular orientation) . . . . . . . . . . . . . . . . 31

5. Dimensional change of $\mathrm{P}_{3} \mathrm{JHAN}$. . . . . . . . . . . . . . . . 41

6a. Dimensional changes of needle-coke H-327 graphite from capsule OG-1, parallel orientation . . . . . . . . . . . . . . . . 44

6b. Dimensional changes of needle-coke H-327 graphite from capsule OG-1, perpendicular orientation . . . . . . . . . . . . . 49

7. Dimensional changes in OG-1 graphites . . . . . . . . . . 52

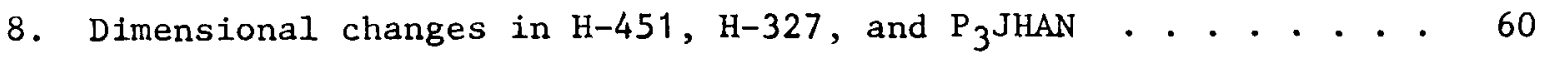

9. Carbon insulating materials irradiated in capsule OG-2 . . . . 61

10. Thermal expansivity of $\mathrm{H}-327$ graphite specimens irradiated in capsule $0 G-1$. . . . . . . . . . . . . . . 63

11. Thermal expansivity of $\mathrm{H}-429$ and $\mathrm{H}-451$ graphite specimens irradiated in capsule $O G-1$. . . . . . . . . . . . . . . . . 64 
12. Thermal conductivity of H-327 graphite specimens irradiated

13. Thermal conductivity of $\mathrm{H}-451$ graphite specimens irradiated

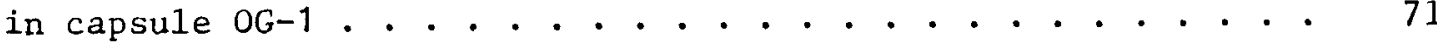

14. Tensile tests on $\mathrm{H}-327$ graphite irradiated in OG-1 capsule . $\quad 80$

15. Tensile tests on H-451 graphite irradiated in OG-1 capsule . $\quad 82$ 
1. INTRODUCTION

This report describes the results of dimensional measurements and physical property tests on graphite samples irradiated in capsule OG-1. Details of the fabrication and assembly of the capsule are given in Ref. 1 , and the operating history of the capsule is described in Ref. 2. A complete list of the samples included in the capsule and their locations is given in Ref. 3 . 


\section{TEST DESCRIPTION AND OBJECTIVES}

Capsule $O G-1$ was the first in a series of graphite irradiation capsules to be irradiated in the $\mathrm{C}-3$ position of the Oak Ridge Reactor (ORR). The two-cell capsule was designed with instrumented temperaturecontrolled crucibles whose operating temperatures spanned the range of temperatures where significant neutron fluence is experienced in a large HTGR $\left(600^{\circ}\right.$ to $\left.1200^{\circ} \mathrm{C}\right)$, with one cell additionally operating at a higher temperature $\left(\sim 1400^{\circ} \mathrm{C}\right)$ to provide test data under conditions which exceed current designs. The fluences ( 1.6 to $3.7 \times 10^{21} \mathrm{n} / \mathrm{cm}^{2}, \mathrm{E}>0.18 \mathrm{MeV}$ ) were chosen so that samples irradiated consecutively in three similar capsules would accumulate data extending beyond end-of-life exposure for current HTGR designs.

The capsule contained 1440 graphite and 70 miscellaneous specimens. A major objective of the experiment was to obtain comparative irradiation data on a needle-coke graphite of the type used in the Fort St. Vrain HTGR (Great Lakes Carbon Company grade H-327) and near-isotropic nuclear graphites of the kind which will be used in future HTGRs. The bulk of the sample space was therefore assigned to $\mathrm{H}-327$ graphite and a production-sized, nearisotropic nuclear graphite made from petroleum coke and its sub-sized prototype (Great Lakes Carbon Company grades $\mathrm{H}-451$ and $\mathrm{H}-429$, respectively). Many of the H-327 and H-429 samples had been previously irradiated in the joint Pacific Northwest Laboratory - General Atomic graphite irradiation capsule GEH-13-422, and a large number of the samples from capsule OG-1 were reloaded into the succeeding capsule, OG-2, to provide higher-fluence data. Other sample space was assigned to graphite types of general interest and to highly irradiated material from previous capsules. 
The capsule contained three types of graphite samples: 0.2-in.diameter by 0.45-in.-long cylinders for dimensional and thermal expansivity measurements, 0.25-in.-diameter by 0.9-in.-long cylinders for dimensional and tensile property measurements, and 0.4-in.-diameter by 0.05-in.-thick discs for thermal diffusivity measurements. A portion of the tensile samples were extended to failure after irradiation, and the remainder were reencapsulated in OG-2. Capsule OG-1 also contained 0.2-in.-diameter by 0.9-in.-long cylinders of $\mathrm{H}-451$ graphite, which are being held for fatigue testing at a later date. 


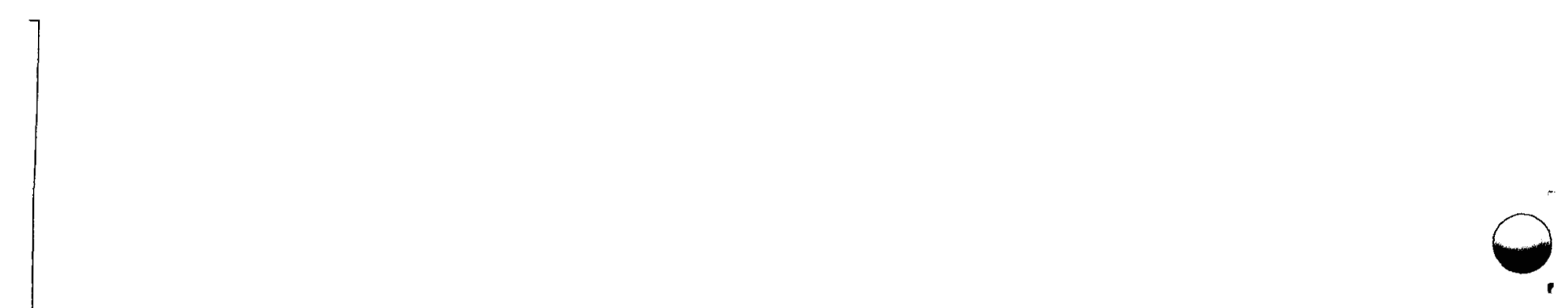

3. MATERIALS INVESTIGATED

The materials investigated are listed in Table 1. 
TABLE 1

GRAPHITES AND MISCELLANEOUS MATERIALS IN OG- 1

\begin{tabular}{|c|c|c|c|c|c|c|c|}
\hline \multirow[b]{2}{*}{ Designation } & \multirow[b]{2}{*}{ Type } & \multirow{2}{*}{$\begin{array}{l}\text { Log } \\
\text { Book Number }\end{array}$} & \multirow{2}{*}{$\begin{array}{l}\text { Form } \& \text { Dimensions } \\
\text { of Parent Sample }\end{array}$} & \multirow[b]{2}{*}{ Source ${ }^{(a)}$} & \multicolumn{3}{|c|}{ Raw Materials } \\
\hline & & & & & Filler & Binder & Impregnant \\
\hline \multicolumn{8}{|c|}{ Graphite } \\
\hline$H-451$ & $\begin{array}{l}\text { Extruded } \\
\text { near-1sotropic }\end{array}$ & $5651-28$ & $\begin{array}{l}\text { 18-in.-diameter x 34-in.-long } \\
\log \end{array}$ & GLCC & $\begin{array}{l}\text { Near-isotropic } \\
\text { petroleum coke }\end{array}$ & $\begin{array}{l}\text { Coal tar } \\
\text { pitch }\end{array}$ & $\begin{array}{l}\text { Petroleum } \\
\text { pitch }\end{array}$ \\
\hline $\mathrm{H}-327$ & $\begin{array}{l}\text { Extruded } \\
\text { anisotropic }\end{array}$ & $4974-3$ & $\begin{array}{l}\text { 18-in.-diameter x 34-in.-1ong } \\
\log \end{array}$ & GLCC & Needle coke & $\begin{array}{l}\text { Coal tar } \\
\text { pitch }\end{array}$ & Coal tar pitch \\
\hline H-429 & $\begin{array}{l}\text { Extruded } \\
\text { near-1sotropic }\end{array}$ & 4974-104-A & $\begin{array}{l}\text { 6.9-in.-diameter } \times 8-1 n .-10 n g \\
\log \end{array}$ & GLCC & $\begin{array}{l}\text { Near-isotropic } \\
\text { petroleum coke }\end{array}$ & $\begin{array}{l}\text { Coal tar } \\
\text { pitch }\end{array}$ & Coal tar pitch \\
\hline 2020 & $\begin{array}{l}\text { Isostatic molded } \\
\text { fine-grained }\end{array}$ & $5651-56$ & $\begin{array}{l}\text { 18-in.-diameter x 6-in.-long } \\
\text { slab }\end{array}$ & SCCP & Petroleum coke & (b) & (b) \\
\hline $\mathrm{P}_{3}$ JHAN & $\begin{array}{l}\text { Extruded } \\
\text { near-isotropic }\end{array}$ & $5651-53$ & 8-in. $\times$ 8-in. $\times$ 8-in. cube & Pechiney & $\begin{array}{l}\text { Near-isotropic } \\
\text { coal tar pitch } \\
\text { coke }\end{array}$ & $\begin{array}{l}\text { Coal tar } \\
\text { pitch }\end{array}$ & Coal tar pitch \\
\hline$H-453$ & $\begin{array}{l}\text { Extruded } \\
\text { near-isotropic }\end{array}$ & $5651-34$ & $\begin{array}{l}\text { 18-in.-diameter x 34-in.-long } \\
\text { half-log }\end{array}$ & GLCC & $\begin{array}{l}\text { Near-isotropic } \\
\text { petroleum coke }\end{array}$ & $\begin{array}{l}\text { Coal tar } \\
\text { pitch }\end{array}$ & $\begin{array}{l}\text { Petroleum } \\
\text { pitch }\end{array}$ \\
\hline $\mathrm{H}-419$ & $\begin{array}{l}\text { (b) } \\
\text { near-isotropic }\end{array}$ & & $\begin{array}{l}1.25 \text {-in.-diameter } \times 5 \text {-in.-long } \\
\text { rod }\end{array}$ & GLCC & (b) & (b) & (b) \\
\hline $\mathrm{H}-315 \mathrm{~A}$ & $\begin{array}{l}\text { BKR } \\
\text { near-1sotropic }\end{array}$ & & $\begin{array}{l}4.6-i n . \text { OD } \times 3.5-i n . ~ I D \\
\text { tube }\end{array}$ & GLCC & Gilsocoke & $\begin{array}{l}\text { Coal tar } \\
\text { pitch }\end{array}$ & Coal tar pitch \\
\hline $\mathrm{H}-328$ & $\begin{array}{l}\text { Molded } \\
\text { near-isotropic }\end{array}$ & & $\begin{array}{l}\text { 17-in.-diameter x 34-in.-long } \\
\text { log }\end{array}$ & GLCC & Gilsocoke & $\begin{array}{l}\text { Coal tar } \\
\text { pitch }\end{array}$ & Coal tar pitch \\
\hline CHN & $\begin{array}{l}\text { Extruded } \\
\text { anisotropic }\end{array}$ & & $\begin{array}{l}\text { 4.6-in.-diameter x 12-in.-long } \\
\log \end{array}$ & UCC & Needle coke & $\begin{array}{l}\text { Coal tar } \\
\text { pitch }\end{array}$ & $\begin{array}{l}\text { Non-graphitizing } \\
\text { carbon }\end{array}$ \\
\hline $\mathrm{H}-319$ & $\begin{array}{l}\text { BKR } \\
\text { near-isotropic }\end{array}$ & & $\begin{array}{l}2.9 \text {-in.-diameter } \times \text { 6-in.-1ong } \\
\log \end{array}$ & GLCC & $\begin{array}{l}\text { Uncalcined } \\
\text { petroleum coke }\end{array}$ & $\begin{array}{l}\text { Coal tar } \\
\text { pitch }\end{array}$ & Coal tar pitch \\
\hline
\end{tabular}


TABLE 1 (Contd)

\begin{tabular}{|c|c|c|c|c|c|c|c|}
\hline \multirow[b]{2}{*}{ Deslgnation } & \multirow[b]{2}{*}{ Type } & \multirow[b]{2}{*}{$\begin{array}{l}\text { Log } \\
\text { Book Number }\end{array}$} & \multirow[b]{2}{*}{$\begin{array}{l}\text { Form \& Dimensions } \\
\text { of Parent Sample }\end{array}$} & \multirow[b]{2}{*}{ Source ${ }^{(a)}$} & \multicolumn{3}{|c|}{ Raw Materials } \\
\hline & & & & & Filler & Binder & Impregnant \\
\hline $11-430$ & $\begin{array}{l}\text { Molded } \\
\text { near-isotropic }\end{array}$ & & $\begin{array}{l}\text { 8.6-in.-diameter } \times 7.5-\text { in.- } \\
\text { long } \log \end{array}$ & GLCC & $\begin{array}{l}\text { Near-isotropic } \\
\text { petroleum coke }\end{array}$ & $\begin{array}{l}\text { Coal tar } \\
\text { pitch }\end{array}$ & (b) \\
\hline $\mathrm{H}-451 \mathrm{C}$ & $\begin{array}{l}\text { Extruded } \\
\text { near-isotropic }\end{array}$ & $5948-14$ & $\begin{array}{l}\text { 18-in.-diameter x } 34 \text {-in.-long } \\
\log \end{array}$ & GLCC/GA & $\begin{array}{l}\text { Near-isotropic } \\
\text { petroleum coke }\end{array}$ & $\begin{array}{l}\text { Coal tar } \\
\text { pitch }\end{array}$ & $\begin{array}{l}\text { Samples taken from } \\
\text { cure-in-place } \\
\text { crucibles }\end{array}$ \\
\hline IH-451 & $\begin{array}{l}\text { Extruded } \\
\text { near-1sotropic }\end{array}$ & $5948-101$ & $\begin{array}{l}\text { 18-in.-diameter x 34-in.-1ong } \\
\log \end{array}$ & GLCC/GA & $\begin{array}{l}\text { Near-isotropic } \\
\text { petroleum coke }\end{array}$ & $\begin{array}{l}\text { Coal tar } \\
\text { pitch }\end{array}$ & $\begin{array}{l}\text { Additional impreg- } \\
\text { nation done at GA }\end{array}$ \\
\hline \multicolumn{8}{|c|}{ Other Materials } \\
\hline & & & - & & \multicolumn{3}{|c|}{ Description } \\
\hline $\mathrm{B}_{4} \mathrm{C}-\mathrm{G}$ & $\begin{array}{l}\text { Extruded } \\
\text { boronated } \\
\text { graphite }\end{array}$ & $5646-13$ & $\begin{array}{l}0.30-i n .-O D \times 10-i n .-10 n g \\
\text { rods }\end{array}$ & GLCC & \multicolumn{3}{|c|}{ Fort St. Vrain lumped burnable poison } \\
\hline GLSYCN & Glassy carbon & (c) & (b) & $\begin{array}{l}\text { Beckwith } \\
\text { Lockheed }\end{array}$ & \multicolumn{3}{|c|}{ Commercial glassy carbons } \\
\hline PYC & Pyrolytic carbon & (c) & (b) & GA & \multicolumn{3}{|c|}{ Restrained and unrestrained LTI PyC } \\
\hline FUSTCK & $\begin{array}{l}\text { Inert fuel } \\
\text { stick }\end{array}$ & $6042-6$ & (b) & GA & \multicolumn{3}{|c|}{$\begin{array}{l}\text { BISO + TRISO inert particles + shim fuel } \\
\text { compacts }\end{array}$} \\
\hline Sic & SiC coatings & $6041-7$ & (b) & GA & \multicolumn{3}{|c|}{ GA-produced SiC coatings } \\
\hline 565161 & Experimental & $5651-61$ & (b) & ORNL & \multicolumn{3}{|c|}{ ORNL experimental graphite } \\
\hline FSVMTX & $\begin{array}{l}\text { Fuel stick } \\
\text { matrix }\end{array}$ & $5478-47$ & (b) & GA & \multicolumn{3}{|c|}{ Reconstituted Fort St. Vrain matrix } \\
\hline KAAE & Pyrolytic carbon & (c) & (b) & GA & \multicolumn{3}{|c|}{ Specialty pyrolytic carbons } \\
\hline
\end{tabular}

(a) GLCC = Great Lakes Carbon Company; UCC = Union Carbide Company; SCCP = Stackpole Carbon Company; GA = General Atomic Company;

OKNL - Oak Ridge National Laboratory.
(b) Unknuwn to author.
(c) Gi experiments on pyrolytic carbon. 


\section{CAPSULE OPERATING CONDITIONS}

\subsection{DESCRIPTION OF CAPSULE}

Capsule OG-1 was a fully instrumented, controlled-temperature graphite irradiation capsule irradiated in the C-3 position of the ORR. Irradiation started on July 7, 1973, and terminated on December 2, 1973 (reactor cycles 113 through 115), for an accumulated irradiation time of 3200 full-power hours.

The capsule contained two cells, each with its own sweep-gas system. Adjustment of the helium-argon ratio in the sweep gas allowed independent temperature control in the upper and lower cells. The cells contained a total of 10 graphite crucibles approximately 2.25 in. in diameter by 2 in. high (except for crucible No. 3, which was $3 \mathrm{in.} \mathrm{high),} \mathrm{which} \mathrm{were} \mathrm{drilled}$ with holes to act as sample holders. The center-line temperatures of crucible 3 in cell 1 and crucible 7 in cell 2 were maintained at $1000^{\circ} \mathrm{C}$ during reactor operation by adjustment of the gas mixtures. The crucibles contained 16 Chromel-Alumel thermocouples and 10 tungsten-rhenium thermocouples. All crucibles had one or more thermocouples located on the center line, and all except two had one or more thermocouples at the periphery. Three sets of in-situ decalibration data on the tungsten-rhenium couples were obtained by locating pairs of Chromel-Alumel and tungsten-rhenium thermocouples in the same holes. The locations of the thermocouples are given in Table 2. Reliable readings were obtained from all thermocouples except $\mathrm{K} 1$ and $\mathrm{W} 2$.

\subsection{OPERATING TEMPERATURES}

Details of the methods used to analyze the thermocouple operating records are given in Ref. 2. The neutron-induced decalibration factor for 
TABLE 2

AVERAGE TEMPERATURE ESTIMATES FOR OG-1 EXPERIMENT

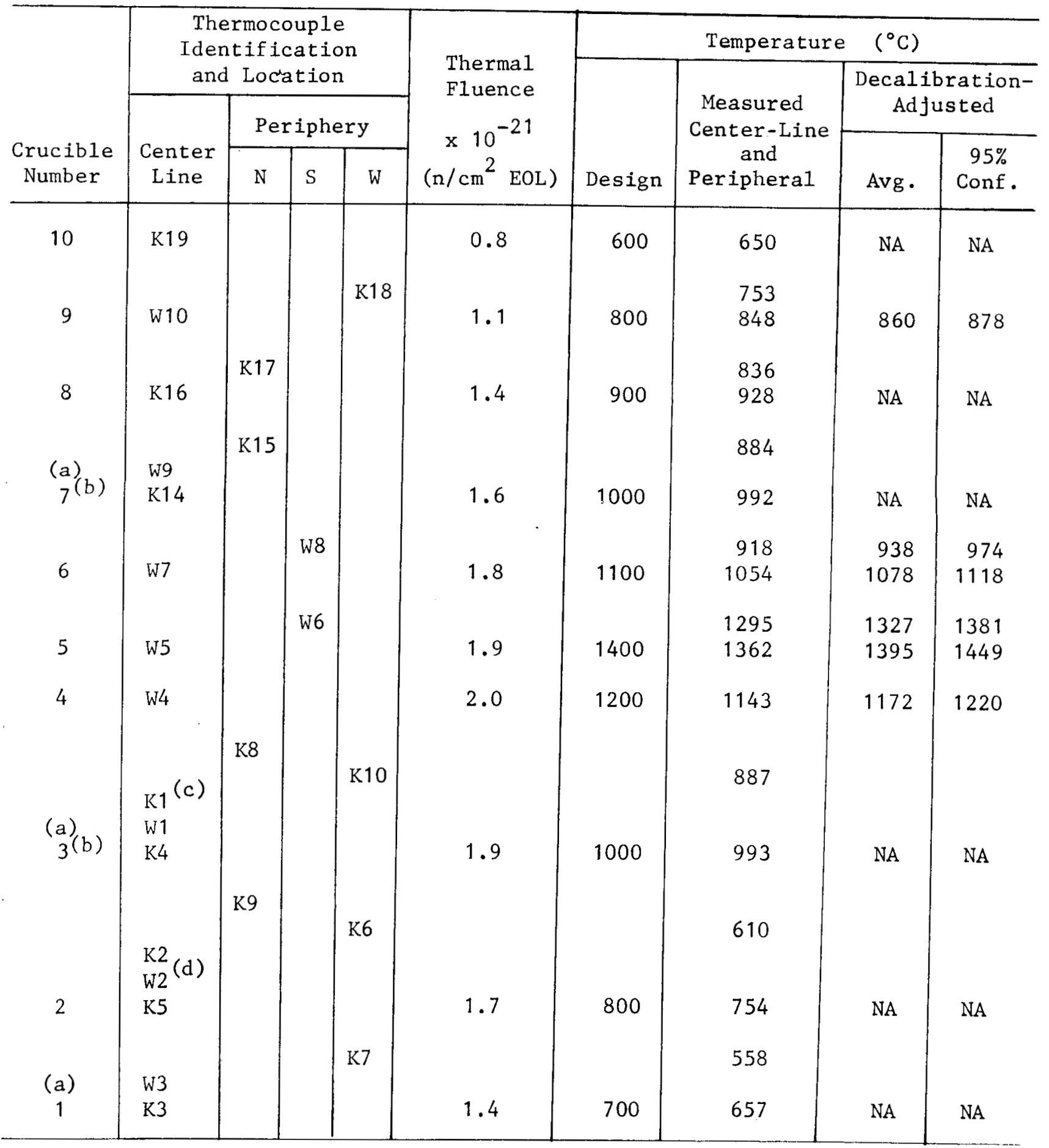

(a) Decalibration estimates obtained from these crucibles.

(b) Contro1 thermocouple.

(c) Data not considered reliable and therefore not used.

(d) Thermocouple did not operate. 
the tungsten-rhenium thermocouples was estimated from the thermocouple pairs located in crucibles 1, 3, and 7 . The following expression for the true operating temperature, $\mathrm{T}_{t}$, in terms of the indicated temperature, $T_{i}$, of the tungsten-rhenium thermocouples was obtained:

$$
T_{t}=\frac{T_{i}}{1-2.45 \times 10^{-23_{f}}} \text {, }
$$

where $\mathrm{f}$ is the thermal fluence $\left(\mathrm{n} / \mathrm{cm}^{2}, \mathrm{E}<0.17 \mathrm{eV}\right)$.

The mean operating temperatures of the thermocouples are shown in Table 2. Tungsten-rhenium thermocouple temperatures corrected according to the above formula are 1isted in the column headed "DecalibrationAdjusted, Avg. " Experimental scatter caused some uncertainty in the decalibration factor; the $95 \%$ upper confidence limits on the decalibrationadjusted temperatures are 1 isted in the last column of the table.

The center-line to periphery temperature differential $(\Delta T)$ for eight of the crucibles was obtained directly from the thermocouple readings. The $\Delta \mathrm{T}$ values for crucibles 4 and 10 were obtained by interpolation from the data for neighboring crucibles, allowing for differences in gamma heating rate. In all crucibles, $\Delta \mathrm{T}$ increased with irradiation owing to the neutron-induced decrease in the thermal conductivity of the graphite. There was a slight long-term drift in the temperatures; over the life of the capsule, the drift in center-line temperatures ranged from $+50^{\circ} \mathrm{C}$ in crucible 4 to $-40^{\circ} \mathrm{C}$ in crucible 9 .

In addition to the long-term drift, normal variations in capsule operating conditions caused fluctuations in the recorded temperatures. The upper limit of the temperature fluctuations (defined as the temperature below which the thermocouple operated for $95 \%$ of the time) and the lower limit (the temperature below which the thermocouple operated for $5 \%$ of the time) were computed from the operating records. 
A summary of the best estimates for the average center-line and peripheral temperatures of each crucible, and the top and bottom of their operating range, appears in Table 3 . The mean operating temperatures of individual samples were estimated from the center-line and peripheral temperatures of the crucible, assuming a parabolic temperature distribution along the radius of the crucible.

\subsection{NEUTRON FLUENCES}

Fast neutron fluences ( $\mathrm{E}>0.18 \mathrm{MeV}$ ) were derived from the activation of iron and titanium dosimeter wires, using activation cross sections of $46.5 \mathrm{mb}$ for the $\mathrm{Fe}^{54}(\mathrm{n}, \mathrm{p}) \mathrm{Mn}^{54}$ reaction and $6.85 \mathrm{mb}$ for the $\mathrm{Ti}^{46}(\mathrm{n}, \mathrm{p}) \mathrm{Sc}^{46}$ reaction. Thermal fluences were measured from vanadium-cobalt dosimeters, assuming a value of $36.8 \mathrm{~b}$ for the cross section for the $\operatorname{Co}^{59}(n, \alpha)$ Co $^{60}$ reaction. The measured fluences are shown as a function of position in the capsule in Fig. 1, and numerical values are listed in Table 3. 
TABLE 3

MEAN TEMPERATURES AND FLUENCES IN CAPSULE OG-1

\begin{tabular}{|c|c|c|c|c|c|c|c|c|}
\hline \multirow[b]{3}{*}{$\begin{array}{l}\text { Crucible } \\
\text { Number }\end{array}$} & \multirow{3}{*}{$\begin{array}{c}\text { Fast } \\
\text { Fluence } \times 10^{-21} \\
\left(\mathrm{n} / \mathrm{cm}^{2}\right) \\
(\mathrm{E}>0.18 \mathrm{MeV})\end{array}$} & \multirow{3}{*}{$\begin{array}{l}\text { Thermal } \\
\text { F1uence } x 10^{-21} \\
\left(\mathrm{n} / \mathrm{cm}^{2}\right)\end{array}$} & \multicolumn{6}{|c|}{ Operating Temperature $\left({ }^{\circ} \mathrm{C}\right)$} \\
\hline & & & \multicolumn{3}{|c|}{ Center Line } & \multicolumn{3}{|c|}{ Periphery } \\
\hline & & & Mean & $\operatorname{Max} .(a)$ & $\operatorname{Min}$ (a) & Mean & $\operatorname{Max} .(a)$ & Min. (a) \\
\hline 1 & 2.2 & 1.4 & 657 & -- & -- & 558 & 578 & 547 \\
\hline 2 & 2.9 & 1.7 & 754 & 789 & 724 & 610 & 624 & 597 \\
\hline 3 & 3.4 & 1.9 & 993 & 1017 & 972 & 887 & 912 & 869 \\
\hline 4 & 3.7 & 2.0 & 1172 & -- & -- & 1024 & 1117 & 962 \\
\hline 5 & 3.6 & 2.0 & 1395 & 1465 & 1347 & 1327 & 1399 & 1271 \\
\hline 6 & 3.4 & 1.8 & 1078 & 1165 & 1052 & 938 & 1043 & 890 \\
\hline 7 & 3.1 & 1.6 & 992 & 1015 & 974 & 884 & 923 & 854 \\
\hline 8 & 2.6 & 1.4 & 928 & 968 & 898 & 836 & 875 & 809 \\
\hline 9 & 2.1 & 1.1 & 860 & 928 & 821 & 753 & 805 & 722 \\
\hline 10 & 1.6 & 0.8 & 650 & 716 & 616 & 597 & 666 & 563 \\
\hline
\end{tabular}

(a) Maximum and minimum temperatures denote the range within which temperatures remained for $90 \%$ of the time the reactor was at power. 


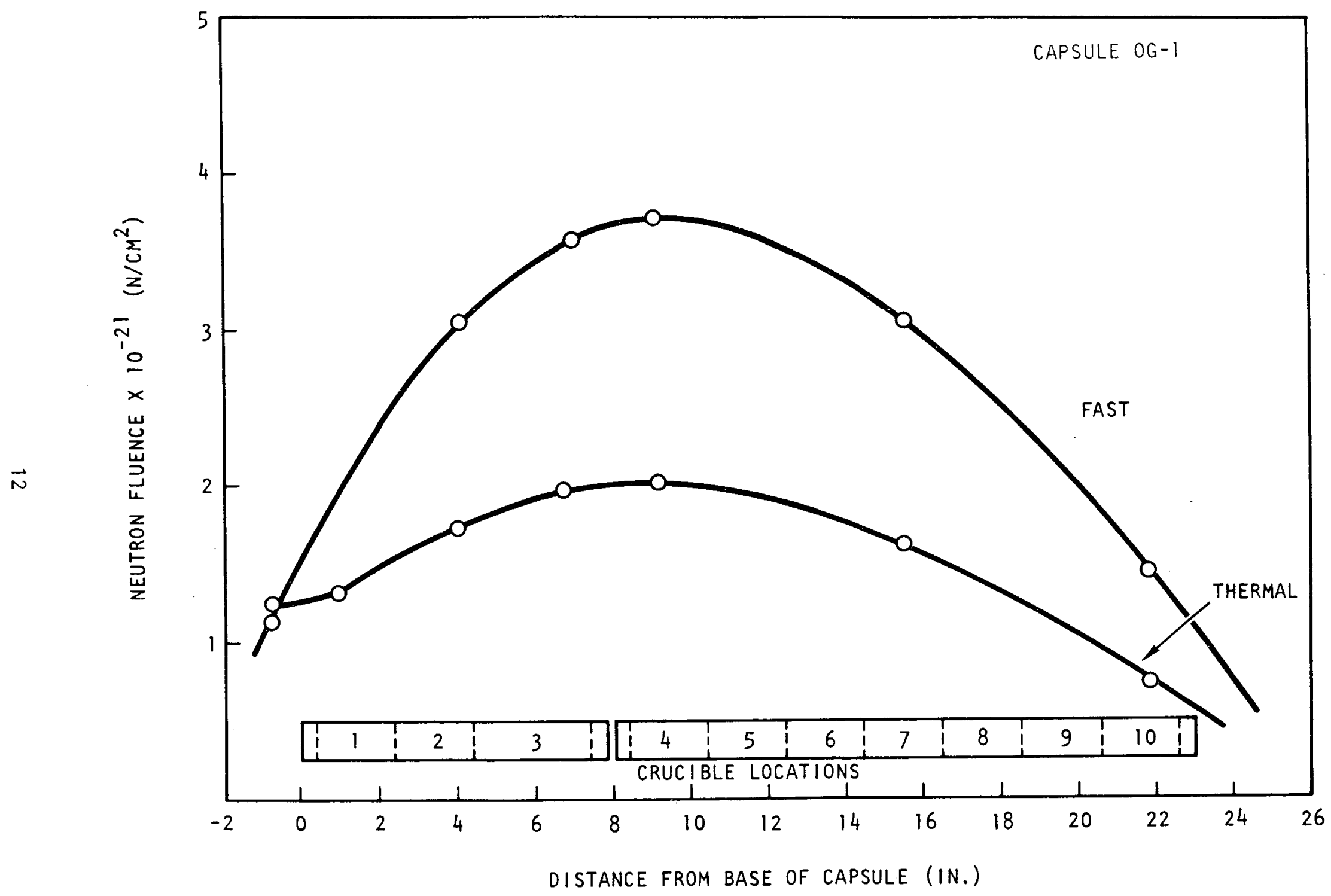

Fig. 1. Fluence as a function of axial position in capsule OG-1 


\section{EXPERIMENTAL RESULTS - DIMENSIONAL CHANGES}

\subsection{IRRADIATION-INDUCED DIMENSIONAL CHANGE IN NEAR-ISOTROPIC GRAPHITES}

Dimensional changes were calculated from the preirradiation and postirradiation lengths measured on $607 \mathrm{H}-451$ graphite cylinders and $70 \mathrm{H}-429$ graphite cylinders. The $\mathrm{H}-429$ specimens were selected from samples previously irradiated in capsule GEH-13-422. Incorporation of the 70 previously irradiated $\mathrm{H}-429$ specimens brought the peak total fluence to $6.7 \times 10^{21} \mathrm{n} / \mathrm{cm}^{2}$ ( $\mathrm{E}>0.18 \mathrm{MeV}$ ) for the near-isotropic graphites irradiated in $O G-1$. The OG-1 dimensional change data are presented in Figs. 2 and 3 , which include hand-drawn dimensional change curves through the data presented in Table 4.

The $\mathrm{H}-451$ and $\mathrm{H}-429$ dimensional change data obtained from OG-1 were combined with previously measured data on H-429 and Gilsocoke graphites and used to generate updated design curves. The dimensional change curves presented in Figs. 4 and 5 are isotherms through a three-dimensional surface obtained by a least-squares fit to the data space defined by dimensional change, fast neutron fluence, and irradiation temperature. The data included 820 data points, 677 from the OG-1 experiment.

The OG-1 samples of $\mathrm{H}-451$ and $\mathrm{H}-429$ exhibited about $20 \%$ less contraction in the parallel direction between $750^{\circ}$ and $1150^{\circ} \mathrm{C}$ than had been predicted from the earlier $\mathrm{H}-429$ and Gilsocoke data. The dimensional changes observed in the perpendicular direction were as predicted. Samples taken from the edge of the graphite $\log$ underwent slightly less ( $1 \%$ to $6 \%$ ) dimensional change than companion samples taken from the center of the log. No systematic variation of dimensional change with sample size was evident in comparisons between 0.45-in. by 0.20-in.-diameter and 0.90-in. by 0.25 -in.-diameter samples. 


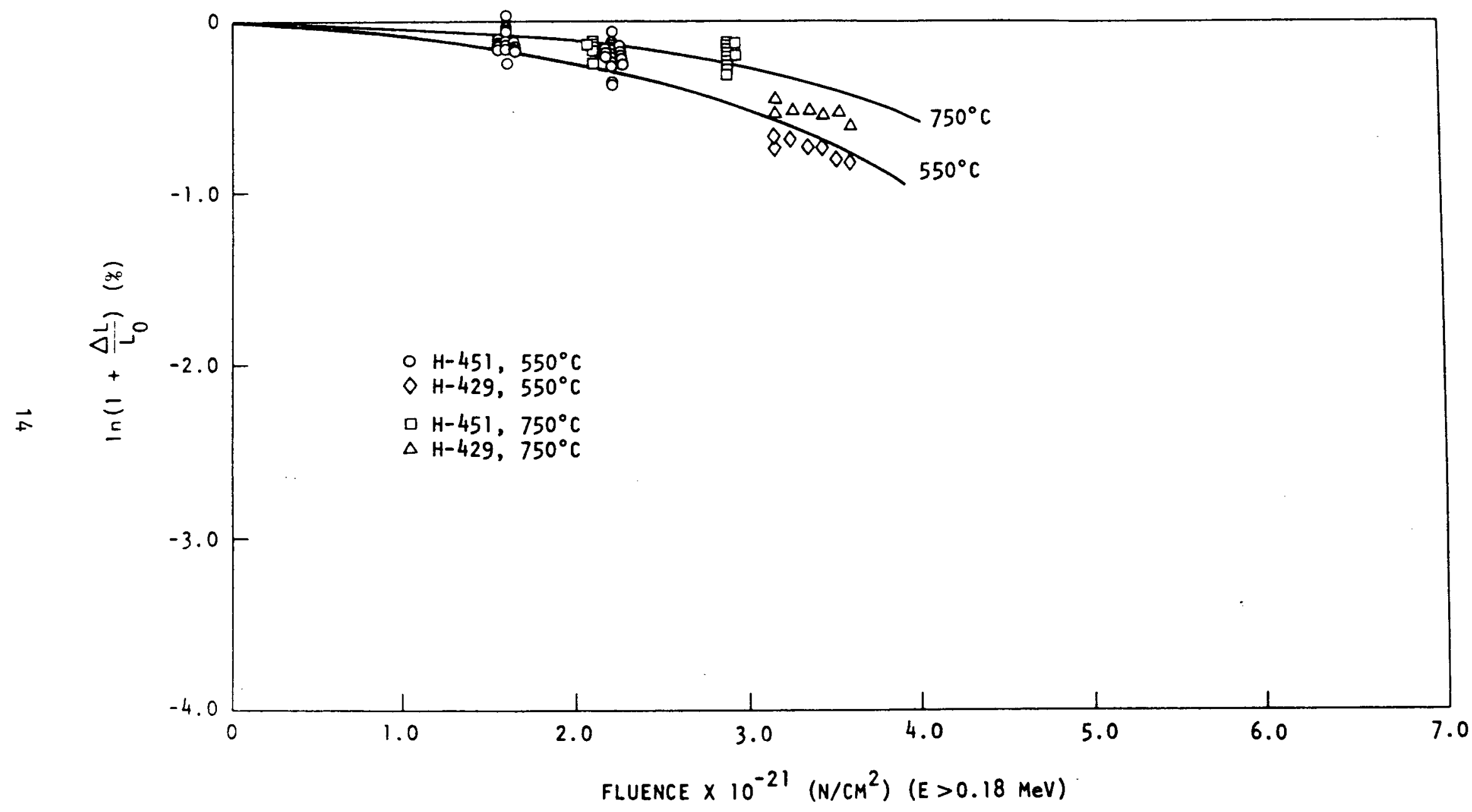

Fig. 2a. Dimensional changes of near-isotropic graphites $\mathrm{H}-451 / \mathrm{H}-429$ in the parallel direction for irradiation temperatures of $550^{\circ}$ and $750^{\circ} \mathrm{C}$ 


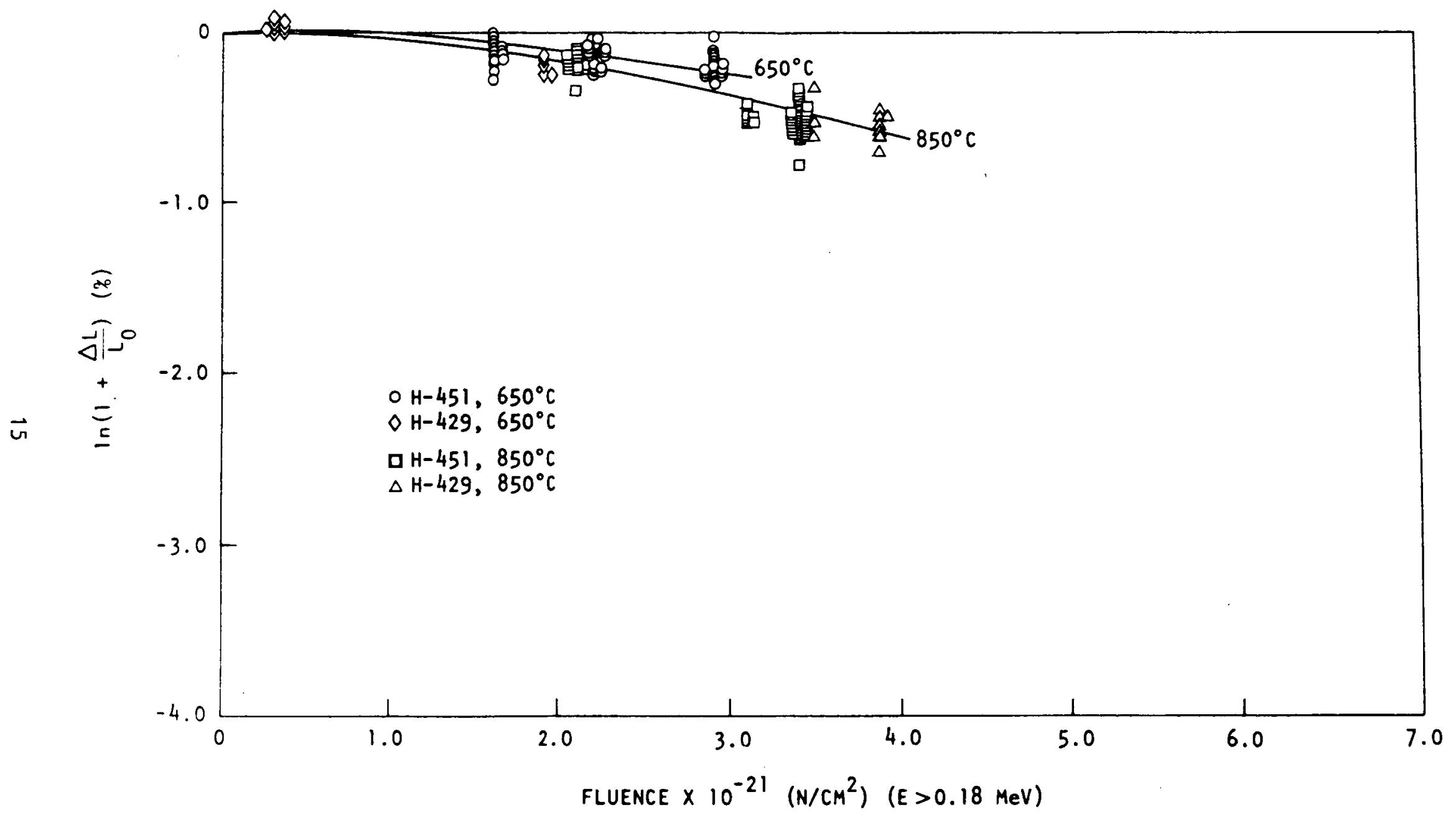

Fig. 2b. Dimensional changes of near-isotropic graphites $H-451 / H-429$ in the parallel direction for irradiation temperatures of $650^{\circ}$ and $850^{\circ} \mathrm{C}$ 


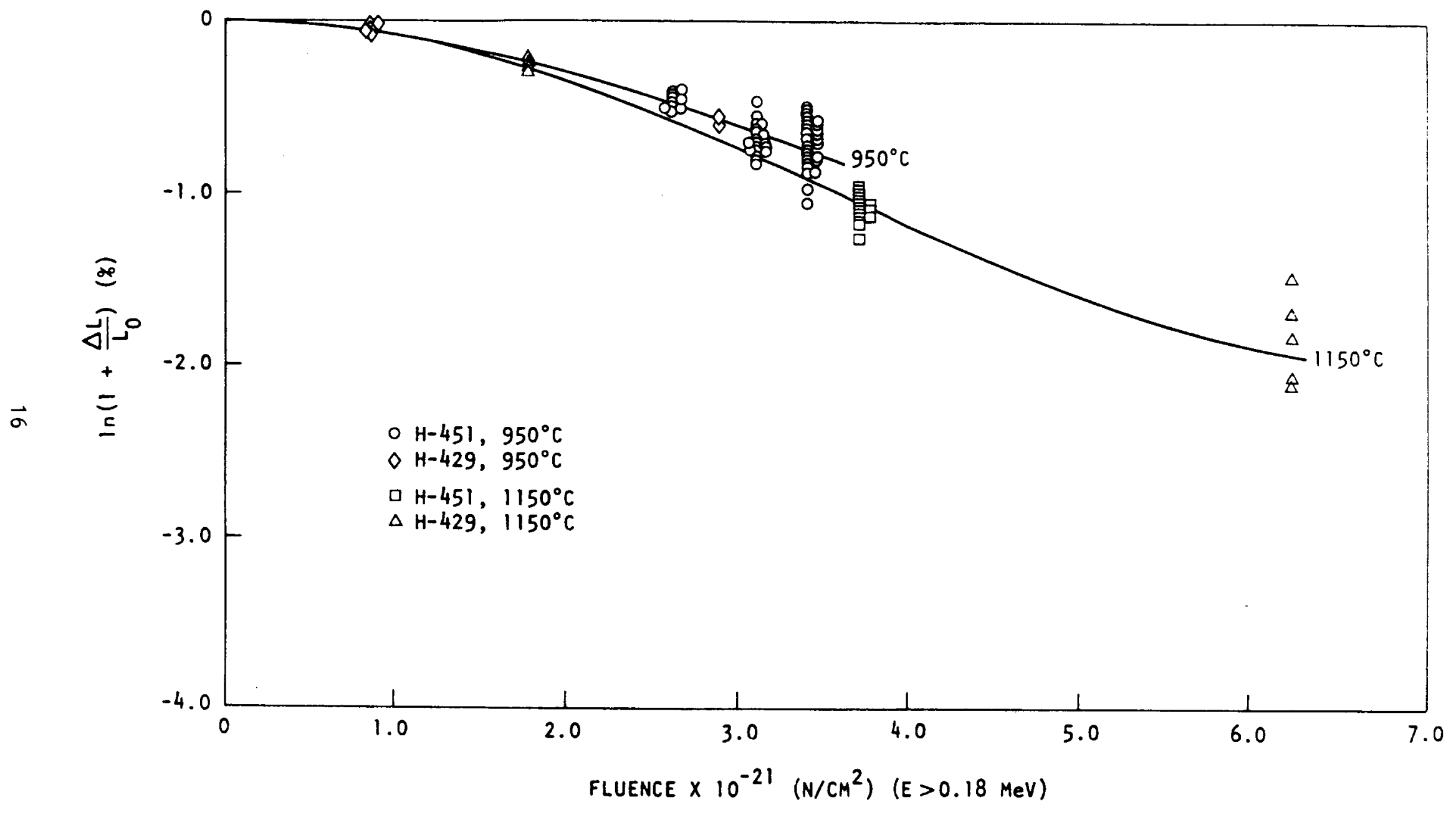

Fig. 2c. Dimensional changes of near-isotropic graphites $H-451 / \mathrm{H}-429$ in the parallel direction for irradiation temperatures of $950^{\circ}$ and $1150^{\circ} \mathrm{C}$ 


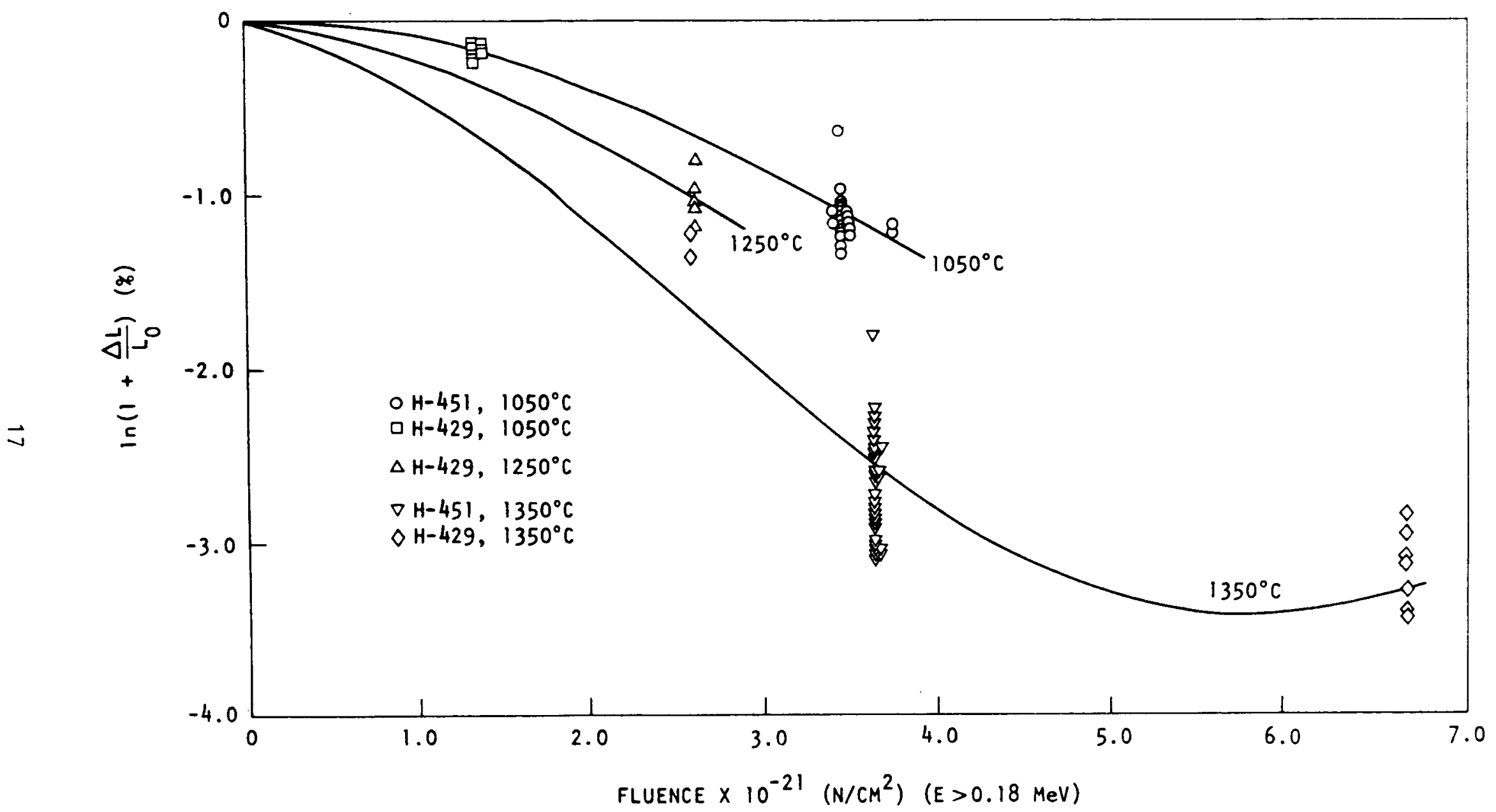

Fig. 2d. Dimensional changes of near-isotropic graphites H-451/H-429 in the parallel direction for irradiation temperatures of $1050^{\circ}, 1250^{\circ}$, and $1350^{\circ} \mathrm{C}$ 


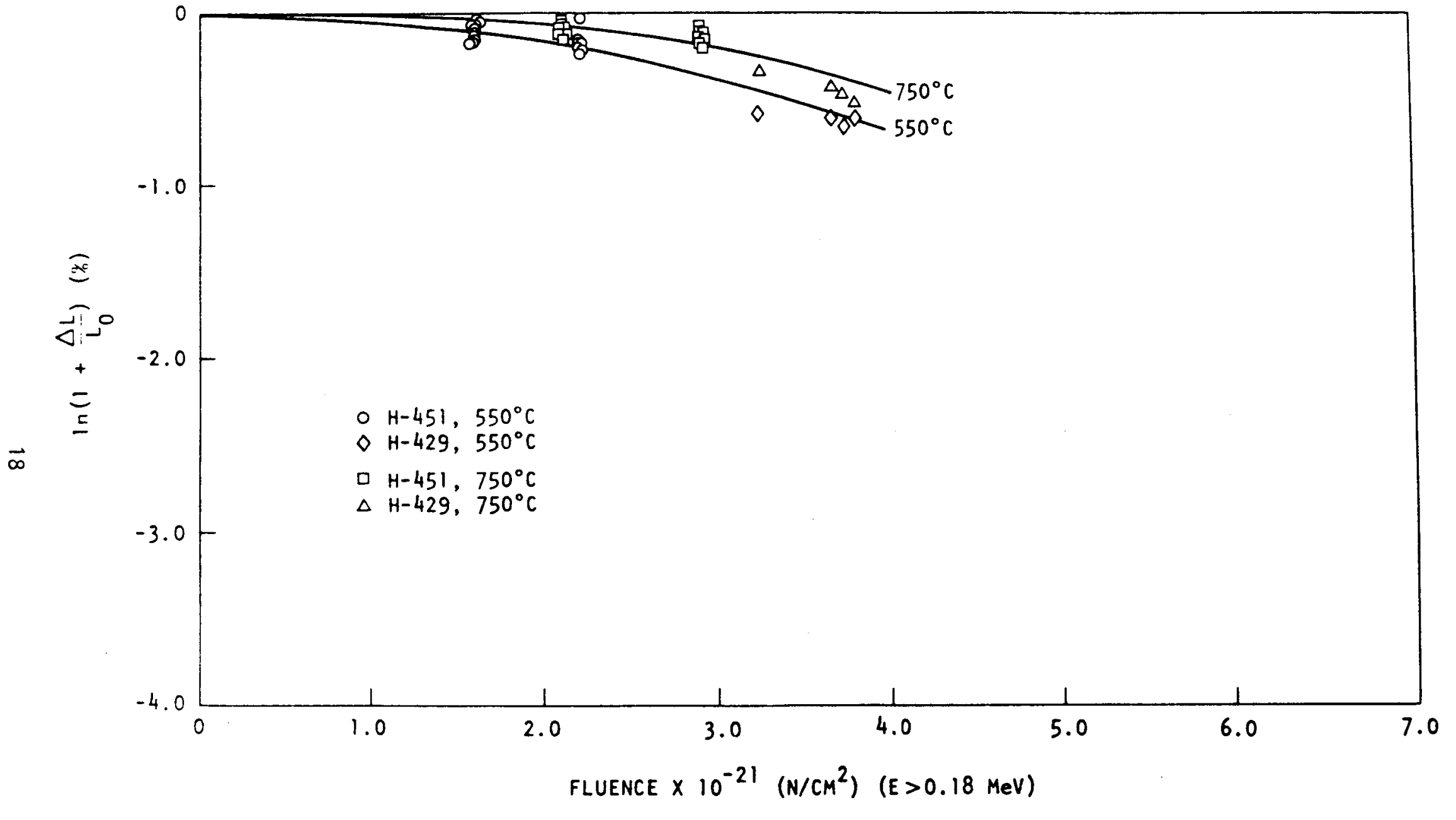

Fig. 3a. Dimensional changes of near-isotropic graphites $\mathrm{H}-451 / \mathrm{H}-429$ in the perpendicular direction for irradiation temperatures of $550^{\circ}$ and $750^{\circ} \mathrm{C}$ 


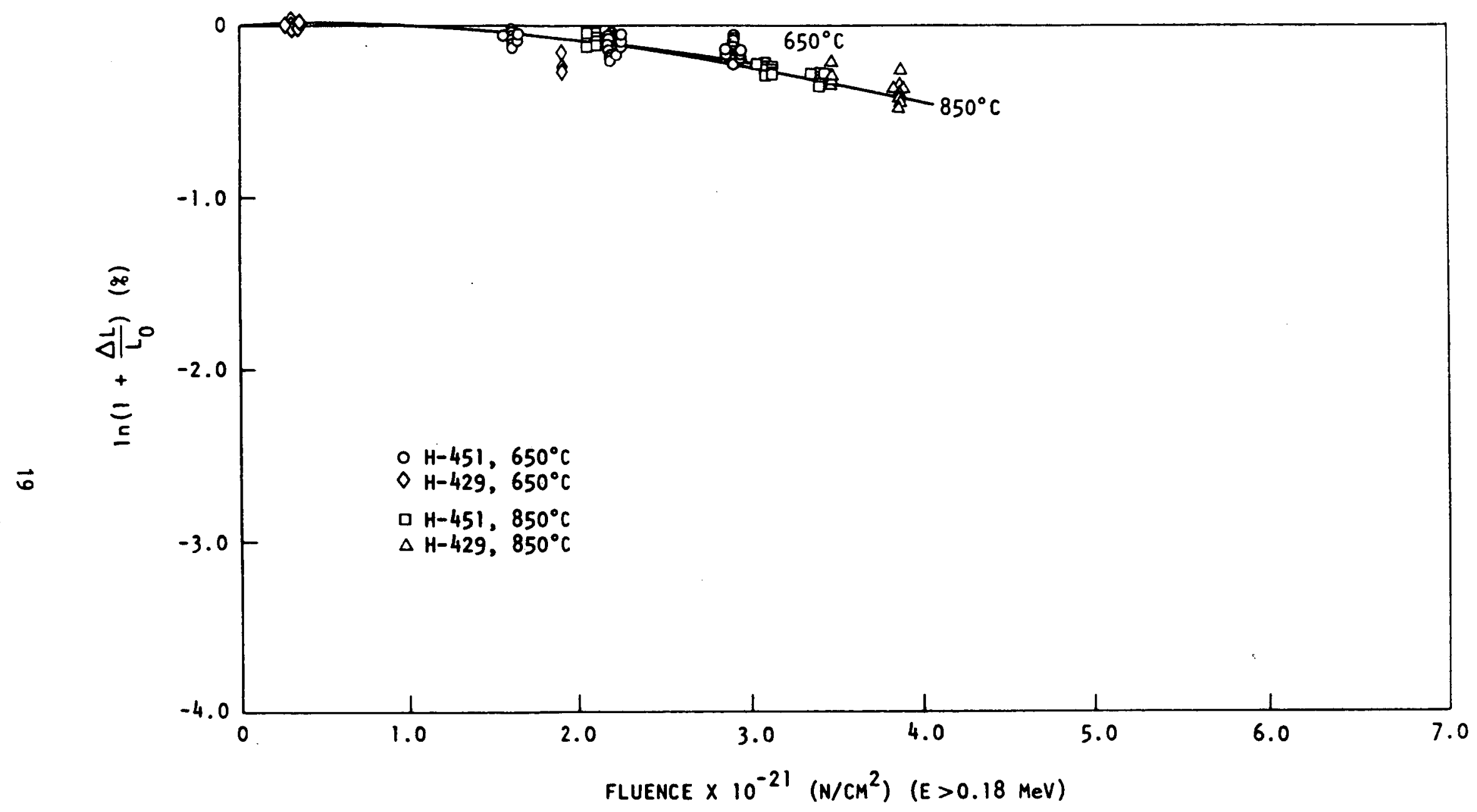

Fig. 3b. Dimensional changes of near-isotropic graphites $\mathrm{H}-451 / \mathrm{H}-429$ in the perpendicular direction for irradiation temperatures of $650^{\circ}$ and $850^{\circ} \mathrm{C}$ 


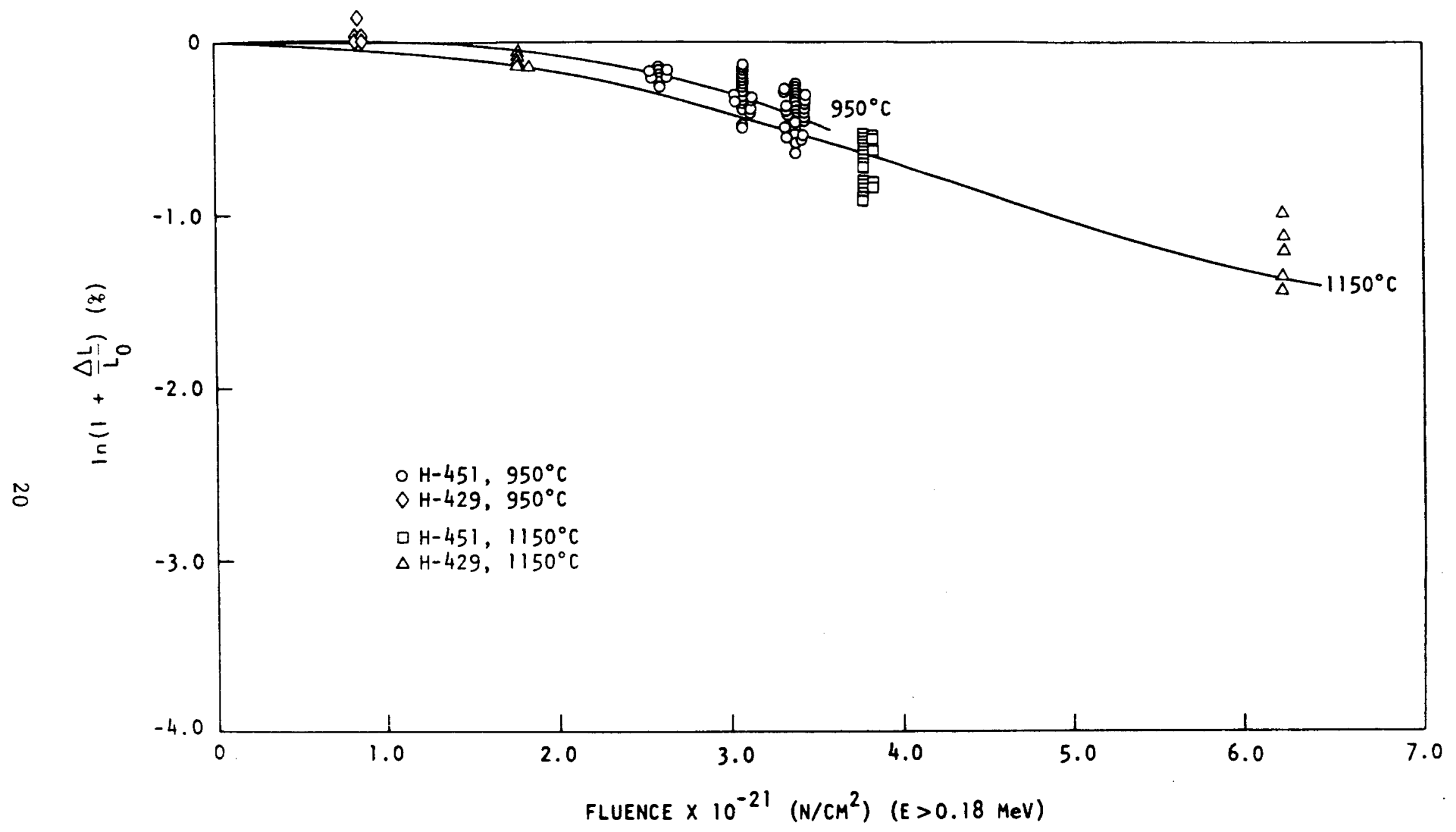

Fig. 3c. Dimensional changes of near-isotropic graphites $\mathrm{H}-451 / \mathrm{H}-429$ in the perpendicular direction for irradiation temperatures of $950^{\circ}$ and $1150^{\circ} \mathrm{C}$ 


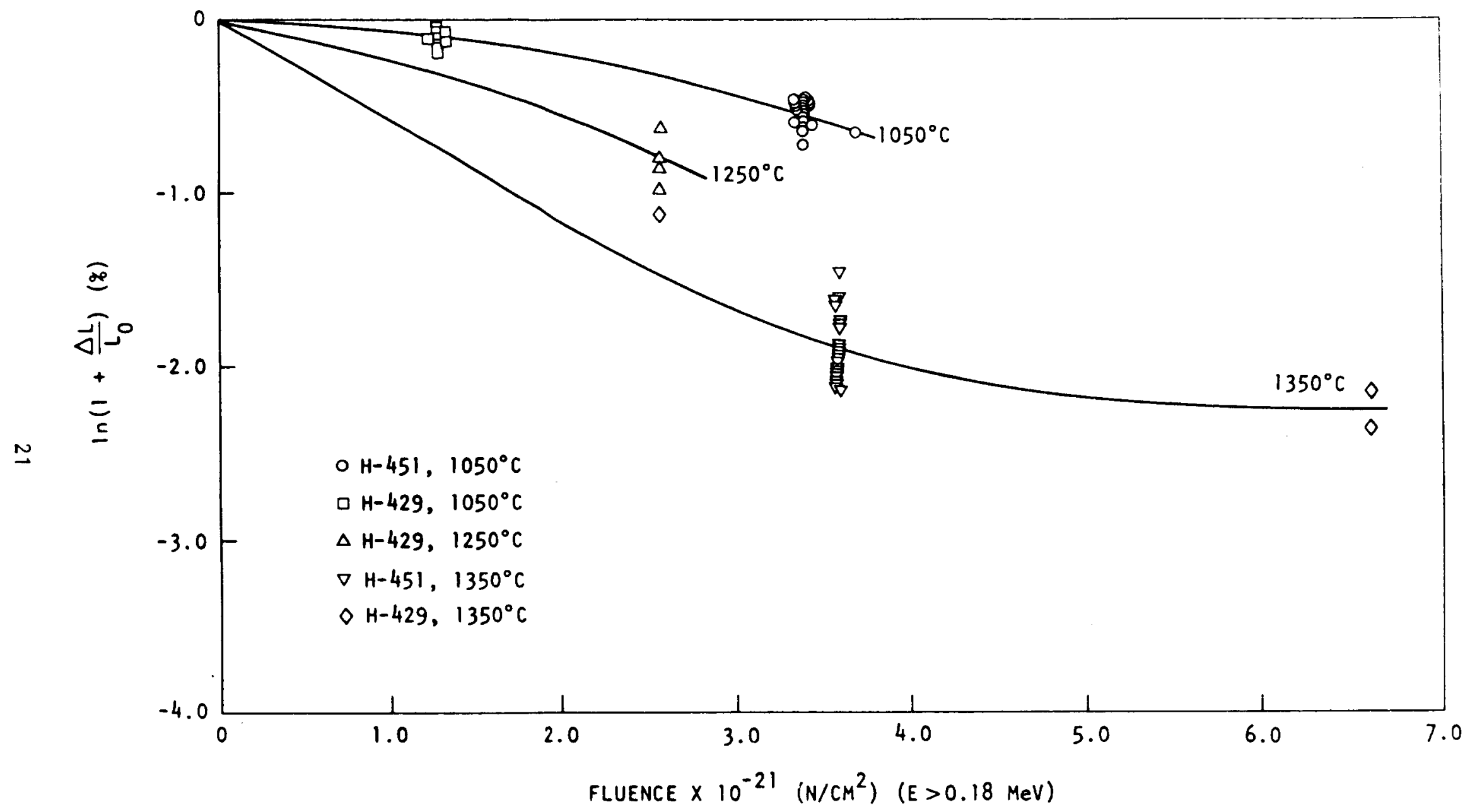

Fig. 3d. Dimensional changes of near-isotropic graphites $\mathrm{H}-451 / \mathrm{H}-429$ in the perpendicular direction for irradiation temperatures of $1050^{\circ}, 1250^{\circ}$, and $1350^{\circ} \mathrm{C}$ 
TABLE $4 \mathrm{~A}$

DIMENSIONAL CHANGE IN H-451 AND H-429 GRAPHITES

(PARALLEL ORIENTATION)

\begin{tabular}{|c|c|c|c|c|}
\hline $\begin{array}{c}\text { Record } \\
\text { No. }\end{array}$ & $\begin{array}{c}\text { Dimensional } \\
\text { Change } \\
\ln \left(1+\frac{\Delta \mathrm{L}}{\mathrm{L}_{\mathrm{o}}}\right) \\
(\%)\end{array}$ & $\begin{array}{l}\text { Average } \\
\text { Irradiation } \\
\text { Temperature } \\
\quad\left({ }^{\circ} \mathrm{C}\right)\end{array}$ & $\begin{array}{c}\text { Total } \\
\text { Fluence } \times 10^{-21} \\
\left(\mathrm{n} / \mathrm{cm}^{2}\right)(\mathrm{E}>0.18 \mathrm{MeV})\end{array}$ & $\begin{array}{l}\text { Previous } \\
\text { Record } \\
\text { No. }\end{array}$ \\
\hline $\begin{array}{l}5000 \\
5001 \\
5004 \\
5008 \\
5012 \\
5016 \\
5020 \\
5024 \\
5028 \\
5032 \\
5036 \\
5040 \\
5044 \\
5045 \\
5048 \\
5049 \\
5050 \\
5051 \\
5054 \\
5055 \\
5058 \\
5059 \\
5060 \\
5061 \\
5064 \\
5065 \\
5068 \\
5069 \\
5090 \\
5091 \\
5092 \\
5093 \\
5096 \\
5097 \\
5100 \\
5101 \\
5102 \\
5103 \\
5104 \\
5105\end{array}$ & $\begin{array}{l}-0.05 \\
-0.10 \\
-0.12 \\
-0.11 \\
-0.10 \\
-0.10 \\
-0.14 \\
-0.14 \\
-0.09 \\
-0.05 \\
-0.12 \\
-0.12 \\
-0.14 \\
-0.19 \\
-0.11 \\
-0.13 \\
-0\end{array}$ & $\mid$ & $3^{2.2}$ & \\
\hline
\end{tabular}


TABLE 4A (Continued)

\begin{tabular}{|c|c|c|c|c|}
\hline $\begin{array}{c}\text { Record } \\
\text { No. }\end{array}$ & $\begin{array}{c}\text { Dimensional } \\
\text { Change } \\
\ln \left(1+\frac{\Delta \mathrm{L}}{\mathrm{L}_{\mathrm{o}}}\right) \\
(\%)\end{array}$ & $\begin{array}{c}\text { Average } \\
\text { Irradiation } \\
\text { Temperature } \\
\left({ }^{\circ} \mathrm{C}\right) \\
\end{array}$ & $\begin{array}{c}\text { Total } \\
\text { F1uence } \times 10^{-21} \\
\left(\mathrm{n} / \mathrm{cm}^{2}\right)(\mathrm{E}>0.18 \mathrm{MeV})\end{array}$ & $\begin{array}{c}\text { Previous } \\
\text { Record } \\
\text { No. } \\
\end{array}$ \\
\hline $\begin{array}{l}5108 \\
5109 \\
5112 \\
5113 \\
5122 \\
5123 \\
5132 \\
5133 \\
5142 \\
5143 \\
5146 \\
5150 \\
5154 \\
5158 \\
5162 \\
5166 \\
5170 \\
5174 \\
5178 \\
6472 \\
5187 \\
5188 \\
5191 \\
5192 \\
5197 \\
5198 \\
5201 \\
5202 \\
5207 \\
5208 \\
5211 \\
5212 \\
5243 \\
5244 \\
5247 \\
5248 \\
5253 \\
5254 \\
5301 \\
5303 \\
5305 \\
5307 \\
5309 \\
5311\end{array}$ & $\begin{array}{l}-0.16 \\
-0.15 \\
-0.16 \\
-0.22 \\
-0.18 \\
-0.14 \\
-0.15 \\
-0.35 \\
-0.14 \\
-0.23 \\
-0.12 \\
-0.15 \\
-0.16 \\
-0.22 \\
-0.28 \\
-0.17 \\
-0.13 \\
-0.12 \\
-0.17 \\
-0.24 \\
-0.16 \\
-0.23 \\
-0\end{array}$ & 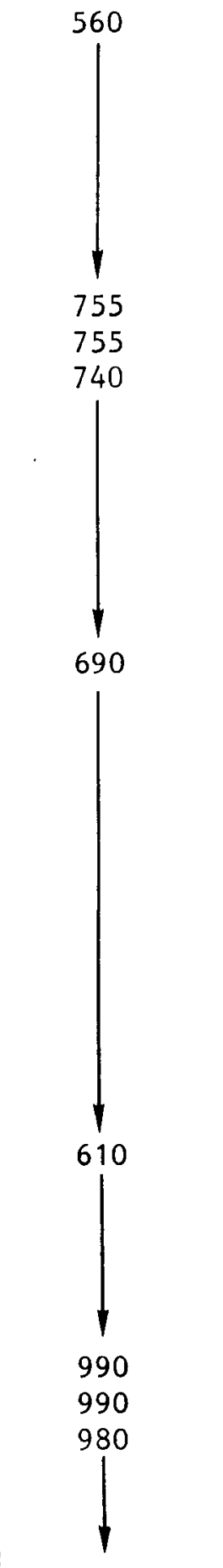 & $\left.\right|^{2}$ & \\
\hline
\end{tabular}


TABLE 4A (Continued)

\begin{tabular}{|c|c|c|c|c|}
\hline $\begin{array}{c}\text { Record } \\
\text { No. }\end{array}$ & $\begin{array}{c}\text { Dimensional } \\
\text { Change } \\
\ln \left(1+\frac{\Delta \mathrm{L}}{\mathrm{L}_{\mathrm{o}}}\right) \\
(\%)\end{array}$ & $\begin{array}{l}\text { Average } \\
\text { Irradiation } \\
\text { Temperature } \\
\quad\left({ }^{\circ} \mathrm{C}\right)\end{array}$ & $\begin{array}{c}\text { Total } \\
\text { Fluence } \times 10^{-21} \\
\left(\mathrm{n} / \mathrm{cm}^{2}\right)(\mathrm{E}>0.18 \mathrm{MeV})\end{array}$ & $\begin{array}{l}\text { Previous } \\
\text { Record } \\
\text { No. }\end{array}$ \\
\hline $\begin{array}{l}5313 \\
5315 \\
5317 \\
5352 \\
5353 \\
5364 \\
5365 \\
5371 \\
5372 \\
5373 \\
5374 \\
5375 \\
5377 \\
5378 \\
5379 \\
5380 \\
5381 \\
5382 \\
5383 \\
5384 \\
5422 \\
5423 \\
5425 \\
5426 \\
5427 \\
5428 \\
5429 \\
5430 \\
5431 \\
5432 \\
5434 \\
5435 \\
5436 \\
5437 \\
5438 \\
5439 \\
5440 \\
5441 \\
5443 \\
5444 \\
5445 \\
5446 \\
5447 \\
5448 \\
5449 \\
5450\end{array}$ & $\begin{array}{l}-0.61 \\
-0.79 \\
-0.99 \\
-0.68 \\
-0.86 \\
-0.61 \\
-0.86 \\
-0.56 \\
-0.74 \\
-0.78 \\
-0.59 \\
-0.77 \\
-0.59 \\
-0.82 \\
-0.51 \\
-0.60 \\
-0.80 \\
-0.83 \\
-0.67 \\
-0.85 \\
-0.50 \\
-0.63 \\
-0.50 \\
-0.57 \\
-0 \\
-0.57 \\
-0.48 \\
-0.60 \\
-0\end{array}$ & 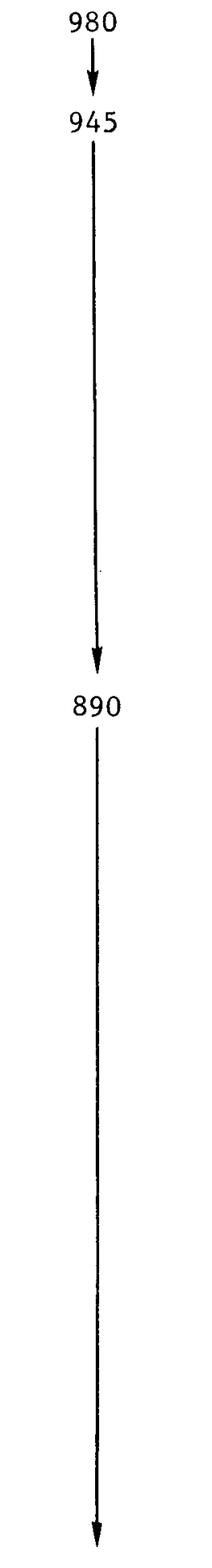 & 4 & \\
\hline
\end{tabular}


TABLE 4A (Continued)

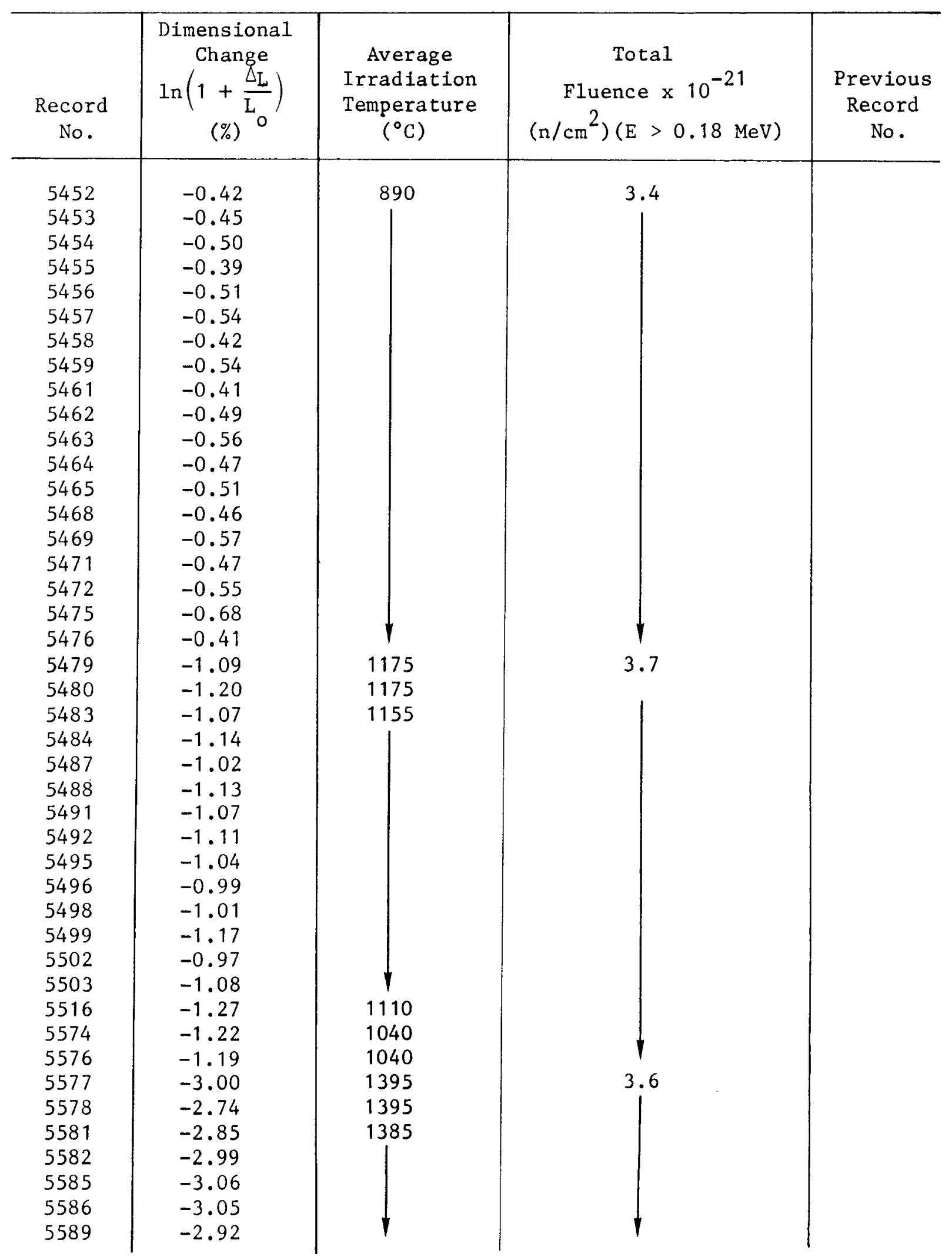


TABLE 4A (Continued)

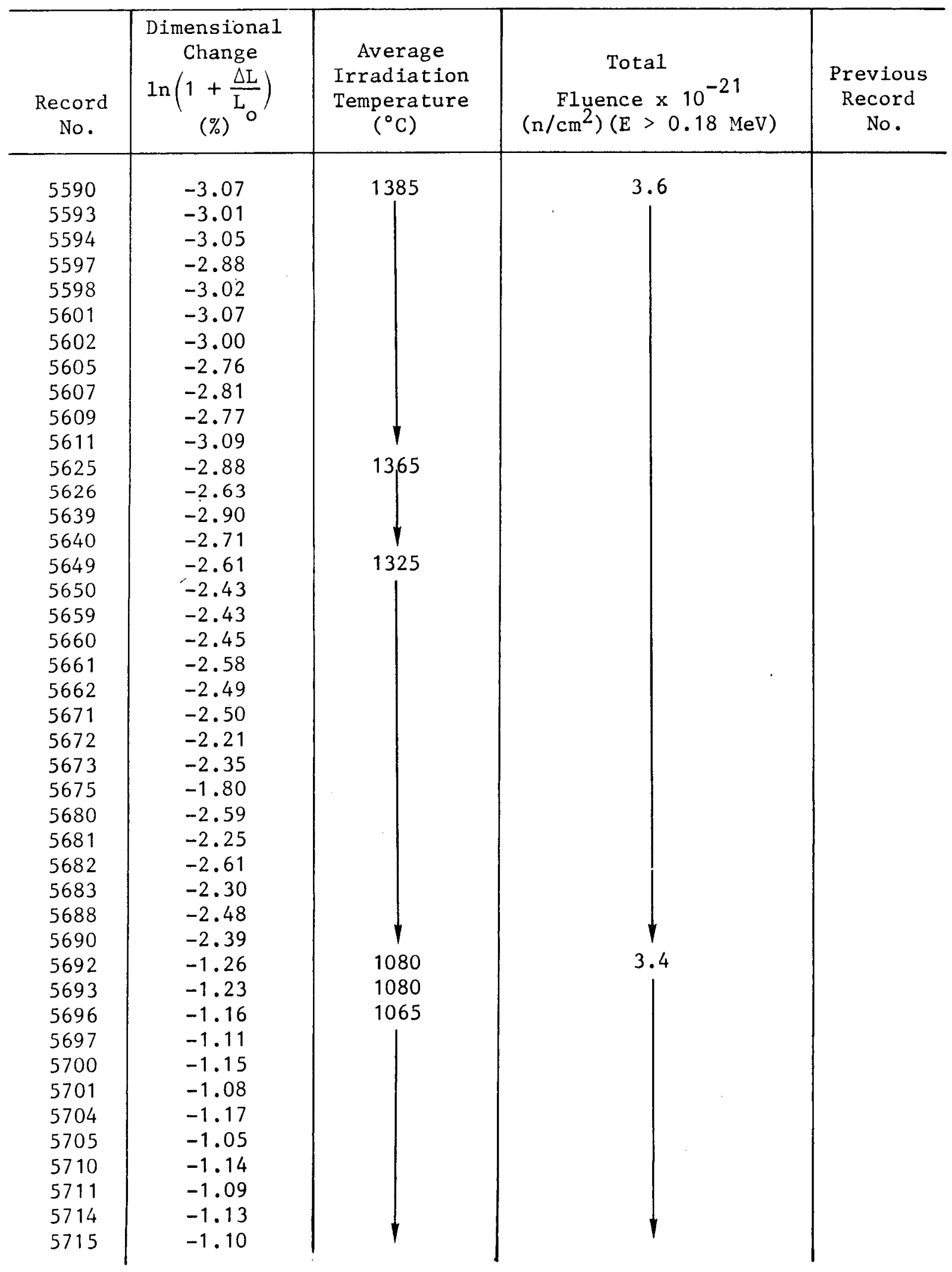


TABLE 4A (Continued)

\begin{tabular}{|c|c|c|c|c|}
\hline $\begin{array}{c}\text { Record } \\
\text { No. }\end{array}$ & $\begin{array}{c}\text { Dimensional } \\
\text { Change } \\
\ln \left(1+\frac{\Delta L}{L_{0}}\right) \\
(\%)\end{array}$ & $\begin{array}{l}\text { Average } \\
\text { Irradiation } \\
\text { Temperature } \\
\quad\left({ }^{\circ} \mathrm{C}\right)\end{array}$ & $\begin{array}{c}\text { Total } \\
\text { Fluence } \times 10^{-21} \\
\left(\mathrm{n} / \mathrm{cm}^{2}\right)(\mathrm{E}>0.18 \mathrm{MeV})\end{array}$ & $\begin{array}{l}\text { Previous } \\
\text { Record } \\
\text { No. }\end{array}$ \\
\hline $\begin{array}{l}5718 \\
5719 \\
5722 \\
5723 \\
5726 \\
5727 \\
5730 \\
5731 \\
5764 \\
5773 \\
5777 \\
5781 \\
5782 \\
5786 \\
5788 \\
5789 \\
5793 \\
5795 \\
5799 \\
5801 \\
5803 \\
5810 \\
5814 \\
5818 \\
5822 \\
5826 \\
5827 \\
5830 \\
5831 \\
5834 \\
5835 \\
5838 \\
5839 \\
5842 \\
5843 \\
5846 \\
5847 \\
5850 \\
5852 \\
5854 \\
5868 \\
5879 \\
5889 \\
5891\end{array}$ & $\begin{array}{l}-1.13 \\
-1.08 \\
-1.17 \\
-1.06 \\
-1.12 \\
-1.03 \\
-1.10 \\
-0.97 \\
-0.63 \\
-0.57 \\
-0.54 \\
-0.50 \\
-0.68 \\
-0.61 \\
-0.50 \\
-0.67 \\
-0.62 \\
-0.70 \\
-0 \\
-0.72 \\
-0.66 \\
-0.81 \\
-0 \\
-0.69 \\
-0.73\end{array}$ & $\mid$ & $\left.\right|^{3.4}$ & \\
\hline
\end{tabular}


TABLE 4A (Continued)

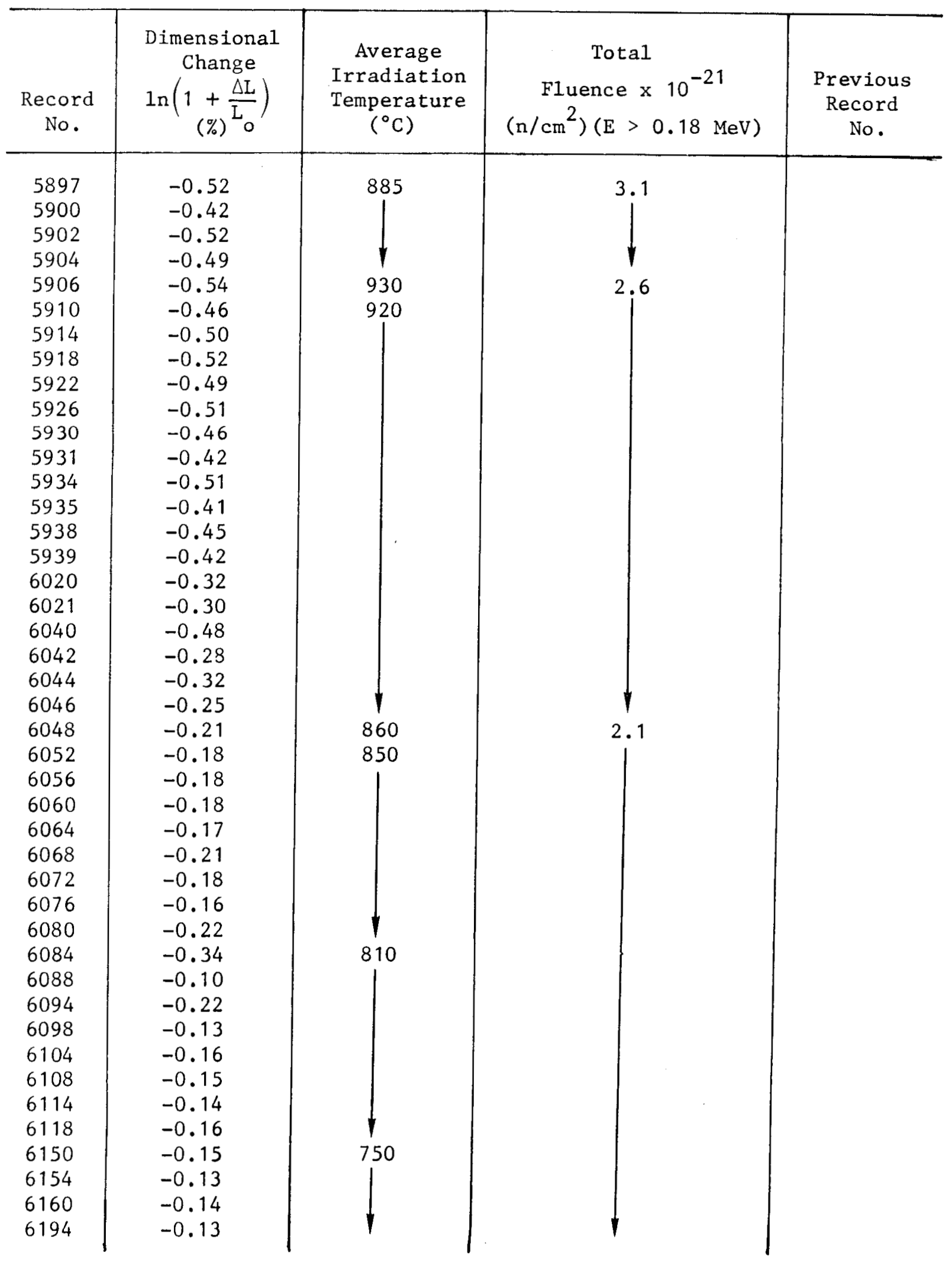


TABLE 4A (Continued)

\begin{tabular}{|c|c|c|c|c|}
\hline $\begin{array}{c}\text { Record } \\
\text { No. }\end{array}$ & $\begin{array}{c}\text { Dimensional } \\
\text { Change } \\
\ln \left(1+\frac{\Delta \mathrm{L}}{\mathrm{L}_{\mathrm{o}}}\right) \\
(\%)\end{array}$ & $\begin{array}{l}\text { Average } \\
\text { Irradiation } \\
\text { Temperature } \\
\quad\left({ }^{\circ} \mathrm{C}\right)\end{array}$ & $\begin{array}{c}\text { Total } \\
\text { Fluence } x 10^{-21} \\
\left(\mathrm{n} / \mathrm{cm}^{2}\right)(E>0.18 \mathrm{MeV})\end{array}$ & $\begin{array}{l}\text { Previous } \\
\text { Record } \\
\text { No. }\end{array}$ \\
\hline $\begin{array}{l}6195 \\
6200 \\
6201 \\
6204 \\
6205 \\
6208 \\
6212 \\
6216 \\
6220 \\
6224 \\
6228 \\
6232 \\
6236 \\
6240 \\
6256 \\
6257 \\
6266 \\
6267 \\
6276 \\
6277 \\
6298 \\
6299 \\
6300 \\
6304 \\
6305 \\
6308 \\
6309 \\
6310 \\
6311 \\
6312 \\
6313 \\
6316 \\
6317 \\
6320 \\
6321 \\
6322 \\
6323 \\
6326 \\
6327 \\
6330 \\
6331 \\
6340 \\
6341\end{array}$ & $\begin{array}{l}-0.14 \\
-0.12 \\
-0.23 \\
-0.14 \\
-0.16 \\
-0.06 \\
-0.04 \\
+0.0 \\
-0.07 \\
-0.10 \\
-0.07 \\
-0.10 \\
-0.22 \\
-0.08 \\
-0.16 \\
-0.13 \\
-0.12 \\
-0.09 \\
-0.11 \\
-0.02 \\
-0.15 \\
-0.06 \\
-0.11 \\
+0.05 \\
-0.05 \\
-0.13 \\
-0.13 \\
-0.14 \\
-0.03 \\
-0.12 \\
-0.11 \\
-0.14 \\
-0.13 \\
-0.13 \\
-0.10 \\
-0.13 \\
-0.16 \\
-0.15 \\
-0.12 \\
-0.15 \\
-0.12 \\
-0.23 \\
-0.12\end{array}$ & $\left.\right|_{150} ^{750}$ & $\left.\right|_{1.6} ^{2.1}$ & \\
\hline
\end{tabular}


TABLE 4A (Continued)

\begin{tabular}{|c|c|c|c|c|}
\hline $\begin{array}{c}\text { Record } \\
\text { No. }\end{array}$ & $\begin{array}{c}\text { Dimensional } \\
\text { Change } \\
\ln \left(1+\frac{\Delta \mathrm{L}}{\mathrm{L}_{0}}\right)\end{array}$ & $\begin{array}{l}\text { Average } \\
\text { Irradiation } \\
\text { Temperature } \\
\quad\left({ }^{\circ} \mathrm{C}\right)\end{array}$ & $\begin{array}{c}\text { Total } \\
\text { Fluence } \times 10^{-21} \\
\left(\mathrm{n} / \mathrm{cm}^{2}\right)(\mathrm{E}>0.18 \mathrm{MeV})\end{array}$ & $\begin{array}{l}\text { Previous } \\
\text { Record } \\
\text { No. }\end{array}$ \\
\hline $\begin{array}{l}5540 \\
5571 \\
5565 \\
5563 \\
5542 \\
5573 \\
5614 \\
5618 \\
5616 \\
5655 \\
5654 \\
5653 \\
5620 \\
5670 \\
5669 \\
5666 \\
5665 \\
5950 \\
5949 \\
5947 \\
6013 \\
6012 \\
6011 \\
6010 \\
6005 \\
6004 \\
6003 \\
5953 \\
5952 \\
5951 \\
6271 \\
6269 \\
6275 \\
6273\end{array}$ & $\begin{array}{l}-1.86 \\
-2.10 \\
-1.45 \\
-2.14 \\
-1.50 \\
-1.71 \\
-3.12 \\
-3.74 \\
-2.83 \\
-3.29 \\
-2.84 \\
-3.43 \\
-3.52 \\
-2.95 \\
-3.15 \\
-3.40 \\
-3.09 \\
-0.09 \\
-0.61 \\
-0.36 \\
-0.54 \\
-0.46 \\
-0.56 \\
-0\end{array}$ & $\begin{array}{l}1140 \\
1140 \\
1160 \\
1160 \\
1140 \\
1140 \\
1425 \\
\mid \\
1390 \\
1425 \\
1385 \\
1390 \\
1390 \\
890 \\
\mid \\
\\
925 \\
925 \\
625 \\
\mid\end{array}$ & 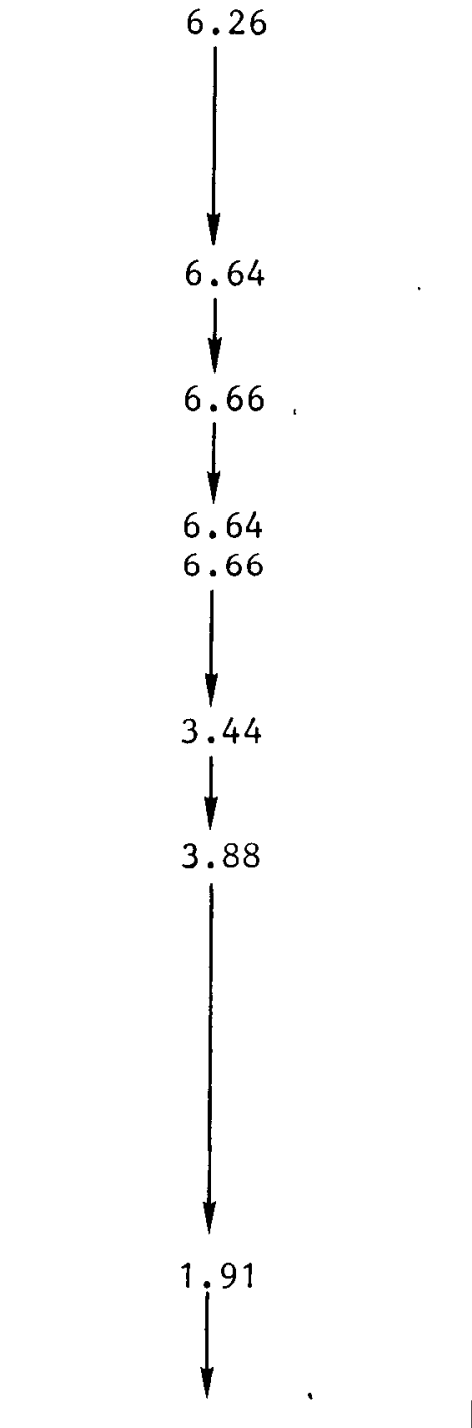 & $\begin{array}{l}2402 \\
2426 \\
2761 \\
2764 \\
2403 \\
2419 \\
2791 \\
2815 \\
2816 \\
2871 \\
2866 \\
2882 \\
2821 \\
2850 \\
2853 \\
2870 \\
2872 \\
2606 \\
2594 \\
2605 \\
2667 \\
2648 \\
2680 \\
2659 \\
2673 \\
2653 \\
2671 \\
2642 \\
2649 \\
2537 \\
2448 \\
2522 \\
2452 \\
2500\end{array}$ \\
\hline
\end{tabular}


TABLE 4B

DIMENSIONAL CHANGE IN H-451 AND H-429 GRAPHITES

(PERPENDICULAR ORIENTATION)

\begin{tabular}{|c|c|c|c|c|}
\hline $\begin{array}{c}\text { Record } \\
\text { No. }\end{array}$ & $\begin{array}{c}\text { Dimensional } \\
\text { Change } \\
\ln \left(1+\frac{\Delta \mathrm{L}}{\mathrm{L}_{\mathrm{o}}}\right) \\
(\%)\end{array}$ & $\begin{array}{l}\text { Average } \\
\text { Irradiation } \\
\text { Temperature } \\
\quad\left({ }^{\circ} \mathrm{C}\right)\end{array}$ & $\begin{array}{c}\text { Total } \\
\text { Fluence } \times 10^{-21} \\
\left(\mathrm{n} / \mathrm{cm}^{2}\right)(\mathrm{E}>0.18 \mathrm{MeV})\end{array}$ & $\begin{array}{l}\text { Previous } \\
\text { Record } \\
\text { No. }\end{array}$ \\
\hline $\begin{array}{l}5002 \\
5003 \\
5006 \\
5010 \\
5014 \\
5018 \\
5022 \\
5026 \\
5030 \\
5034 \\
5038 \\
5042 \\
5046 \\
5047 \\
5052 \\
5053 \\
5056 \\
5057 \\
5062 \\
5063 \\
5066 \\
5067 \\
5094 \\
5095 \\
5098 \\
5099 \\
5106 \\
5107 \\
5110 \\
5111 \\
5144 \\
5145 \\
5148 \\
5152 \\
5156 \\
5160 \\
5164 \\
5168 \\
5172 \\
5176\end{array}$ & $\begin{array}{l}-0.04 \\
-0.06 \\
-0.07 \\
-0.11 \\
-0.10 \\
-0.04 \\
-0.05 \\
-0.10 \\
-0.09 \\
-0.09 \\
-0.19 \\
-0.16 \\
-0.13 \\
-0.18 \\
-0.12 \\
-0.11 \\
-0.14 \\
-0.03 \\
-0.15 \\
-0.08 \\
-0.15\end{array}$ & 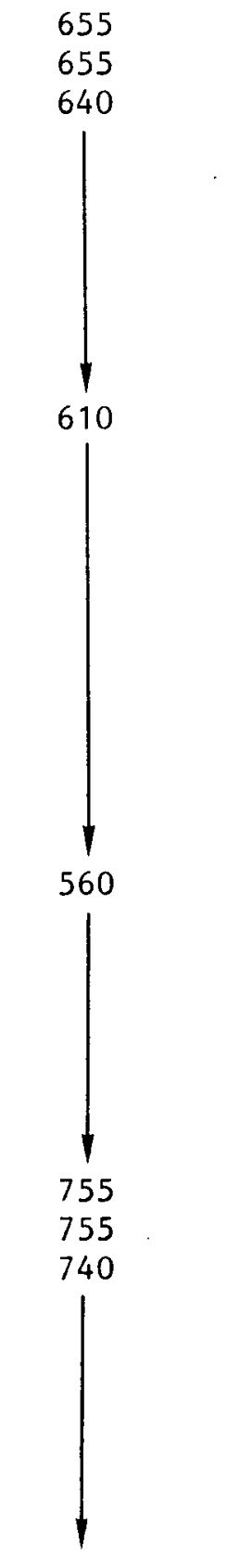 & $\mid$ & \\
\hline
\end{tabular}


TABLE 4B (Continued)

\begin{tabular}{|c|c|c|c|c|}
\hline $\begin{array}{c}\text { Record } \\
\text { No. }\end{array}$ & $\begin{array}{l}\text { Dimensional } \\
\text { Change } \\
\ln \left(1+\frac{\Delta \mathrm{L}}{\mathrm{L}_{\mathrm{o}}}\right) \\
(\%)\end{array}$ & $\begin{array}{l}\text { Average } \\
\text { Irradiation } \\
\text { Temperature } \\
\quad\left({ }^{\circ} \mathrm{C}\right)\end{array}$ & $\begin{array}{c}\text { Total } \\
\text { Fluence } \times 10^{-21} \\
\left(\mathrm{n} / \mathrm{cm}^{2}\right)(\mathrm{E}>0.18 \mathrm{MeV})\end{array}$ & $\begin{array}{l}\text { Previous } \\
\text { Record } \\
\text { No. }\end{array}$ \\
\hline $\begin{array}{l}5180 \\
5183 \\
5189 \\
5190 \\
5193 \\
5194 \\
5199 \\
5200 \\
5203 \\
5204 \\
5209 \\
5210 \\
5213 \\
5214 \\
5245 \\
5246 \\
5249 \\
5250 \\
5255 \\
5256 \\
5302 \\
5304 \\
5306 \\
5308 \\
5310 \\
5312 \\
5314 \\
5316 \\
5318 \\
5337 \\
5338 \\
5339 \\
5340 \\
5341 \\
5342 \\
5343 \\
5344 \\
5345 \\
5346 \\
5347 \\
5348 \\
5349 \\
5350\end{array}$ & $\begin{array}{l}-0.05 \\
-0.11 \\
-0.15 \\
-0.19 \\
-0.12 \\
-0.13 \\
-0.10 \\
-0.16 \\
-0.16 \\
-0.18 \\
-0.20 \\
-0.16 \\
-0.19 \\
-0.16 \\
-0.06 \\
-0.16 \\
-0.15 \\
-0.15 \\
-0.14 \\
-0.23 \\
-0\end{array}$ & $\mid$ & \begin{tabular}{|}
$\mid 9$ \\
4 \\
3.4 \\
1
\end{tabular} & \\
\hline
\end{tabular}


TABLE 4B (Continued)

\begin{tabular}{|c|c|c|c|c|}
\hline $\begin{array}{c}\text { Record } \\
\text { No. }\end{array}$ & $\begin{array}{c}\text { Dimensional } \\
\text { Change } \\
\ln \left(1+\frac{\Delta L}{L_{o}}\right) \\
(\%)\end{array}$ & $\begin{array}{l}\text { Average } \\
\text { Irradiation } \\
\text { Temperature } \\
\quad\left({ }^{\circ} \mathrm{C}\right)\end{array}$ & $\begin{array}{c}\text { Total } \\
\text { Fluence } \times 10^{-21} \\
\left(\mathrm{n} / \mathrm{cm}^{2}\right)(\mathrm{E}>0.18 \mathrm{MeV})\end{array}$ & $\begin{array}{l}\text { Previous } \\
\text { Record } \\
\text { No. }\end{array}$ \\
\hline $\begin{array}{l}5351 \\
5354 \\
5355 \\
5356 \\
5360 \\
5361 \\
5366 \\
5367 \\
5368 \\
5376 \\
5385 \\
5424 \\
5433 \\
5451 \\
5460 \\
5470 \\
5442 \\
5481 \\
5482 \\
5485 \\
5486 \\
5489 \\
5490 \\
5493 \\
5494 \\
5497 \\
5500 \\
5501 \\
6471 \\
5504 \\
5505 \\
5512 \\
5518 \\
5575 \\
5579 \\
5580 \\
5583 \\
5584 \\
5587 \\
5588 \\
5591 \\
5592 \\
5595\end{array}$ & $\begin{array}{l}-0.42 \\
-0.40 \\
-0.31 \\
-0.52 \\
-0.33 \\
-0.36 \\
-0.33 \\
-0.29 \\
-0.41 \\
-0.37 \\
-0 \\
-0.44 \\
-0.37 \\
-0.30 \\
-0.33 \\
-0 \\
-0.31 \\
-0.30 \\
-0.30 \\
-0 \\
-0.81 \\
-0.63\end{array}$ & $\begin{array}{c}945 \\
\mid \\
\mid \\
1175 \\
1175 \\
1155 \\
\mid \\
1110 \\
110 \\
1040 \\
1395 \\
1385 \\
\mid\end{array}$ & $i^{3.4}$ & \\
\hline
\end{tabular}


TABLE 4B (Continued)

\begin{tabular}{|c|c|c|c|c|}
\hline $\begin{array}{c}\text { Record } \\
\text { No. }\end{array}$ & $\begin{array}{c}\text { Dimensional } \\
\text { Change } \\
\ln \left(1+\frac{\Delta \mathrm{L}}{\mathrm{L}_{\mathrm{o}}}\right) \\
(\%)\end{array}$ & $\begin{array}{l}\text { Average } \\
\text { Irradiation } \\
\text { Temperature } \\
\quad\left({ }^{\circ} \mathrm{C}\right)\end{array}$ & $\begin{array}{c}\text { Total } \\
\text { Fluence } \times 10^{-21} \\
\left(\mathrm{n} / \mathrm{cm}^{2}\right)(\mathrm{E}>0.18 \mathrm{MeV})\end{array}$ & $\begin{array}{l}\text { Previous } \\
\text { Record } \\
\text { No. }\end{array}$ \\
\hline $\begin{array}{l}5596 \\
5599 \\
5600 \\
5604 \\
5603 \\
5606 \\
5608 \\
5610 \\
5612 \\
5674 \\
5689 \\
5691 \\
5694 \\
5695 \\
5698 \\
5699 \\
5702 \\
5703 \\
5708 \\
5709 \\
5712 \\
5713 \\
5716 \\
5717 \\
5720 \\
5721 \\
5724 \\
5725 \\
5728 \\
5729 \\
5732 \\
5733 \\
5765 \\
5774 \\
5778 \\
5783 \\
5787 \\
5790 \\
5794 \\
5796 \\
5800 \\
5802 \\
5808\end{array}$ & $\begin{array}{l}-1.94 \\
-2.00 \\
-1.78 \\
-2.03 \\
-2.10 \\
-1.97 \\
-1.64 \\
-2.02 \\
-1.87 \\
-1.60 \\
-1.74 \\
-1.47 \\
-0.60 \\
-0.53 \\
-0.64 \\
-0.49 \\
-0.61 \\
-0.50 \\
-0\end{array}$ & $\mid$ & $\mid$ & \\
\hline
\end{tabular}


TABLE 4B (Continued)

\begin{tabular}{|c|c|c|c|c|}
\hline $\begin{array}{c}\text { Record } \\
\text { No. }\end{array}$ & $\begin{array}{c}\text { Dimensional } \\
\text { Change } \\
\ln \left(1+\frac{\Delta \mathrm{L}}{\mathrm{L}_{\mathrm{o}}}\right) \\
(\%)\end{array}$ & $\begin{array}{l}\text { Average } \\
\text { Irradiation } \\
\text { Temperature } \\
\quad\left({ }^{\circ} \mathrm{C}\right)\end{array}$ & $\begin{array}{c}\text { Total } \\
\text { Fluence } \times 10^{-21} \\
\left(\mathrm{n} / \mathrm{cm}^{2}\right)(\mathrm{E}>0.18 \mathrm{MeV})\end{array}$ & $\begin{array}{l}\text { Previous } \\
\text { Record } \\
\text { No. }\end{array}$ \\
\hline $\begin{array}{l}5812 \\
5816 \\
5820 \\
5824 \\
5828 \\
5829 \\
5832 \\
5833 \\
5836 \\
5837 \\
5840 \\
5841 \\
5844 \\
5845 \\
5848 \\
5849 \\
5851 \\
5853 \\
5855 \\
5869 \\
5880 \\
5890 \\
5892 \\
5898 \\
5899 \\
5901 \\
5903 \\
5905 \\
5908 \\
5912 \\
5916 \\
5920 \\
5924 \\
5928 \\
5932 \\
5933 \\
5936 \\
5937 \\
5940 \\
5941 \\
6022 \\
6023 \\
6041 \\
6043\end{array}$ & $\begin{array}{l}-0.38 \\
-0.41 \\
-0.33 \\
-0.40 \\
-0.51 \\
-0.16 \\
-0.37 \\
-0.42 \\
-0.40 \\
-0.32 \\
-0.34 \\
-0.26 \\
-0.30 \\
-0.14 \\
-0.36 \\
-0.23 \\
-0 \\
-0.50 \\
-0.20 \\
-0.35 \\
-0.31 \\
-0 \\
-0.22 \\
-0.27 \\
-0.24 \\
-0 \\
-0.27 \\
-0.32 \\
-0 \\
-0.25 \\
-0.24 \\
-0 \\
-0.23 \\
-0.18 \\
-0\end{array}$ & $\mid$ & 1 & - \\
\hline
\end{tabular}


TABLE 4B (Continued)

\begin{tabular}{|c|c|c|c|c|}
\hline $\begin{array}{c}\text { Record } \\
\text { No. }\end{array}$ & $\begin{array}{c}\text { Dimensional } \\
\text { Change } \\
\ln \left(1+\frac{\Delta \mathrm{L}}{\mathrm{L}_{\mathrm{o}}}\right) \\
(\%)\end{array}$ & $\begin{array}{l}\text { Average } \\
\text { Irradiation } \\
\text { Temperature } \\
\quad\left({ }^{\circ} \mathrm{C}\right)\end{array}$ & $\begin{array}{c}\text { Total } \\
\text { F1uence } \times 10^{-21} \\
\left(\mathrm{n} / \mathrm{cm}^{2}\right)(\mathrm{E}>0.18 \mathrm{MeV})\end{array}$ & $\begin{array}{l}\text { Previous } \\
\text { Record } \\
\text { No. }\end{array}$ \\
\hline $\begin{array}{l}6047 \\
6045 \\
6050 \\
6054 \\
6058 \\
6062 \\
6066 \\
6070 \\
6074 \\
6078 \\
6082 \\
6086 \\
6090 \\
6096 \\
6100 \\
6106 \\
6110 \\
6116 \\
6120 \\
6152 \\
6156 \\
6162 \\
6196 \\
6197 \\
6202 \\
6203 \\
6206 \\
6207 \\
6210 \\
6214 \\
6218 \\
6222 \\
6226 \\
6230 \\
6234 \\
6238 \\
6242 \\
6302 \\
6306 \\
6307 \\
6314 \\
6315 \\
6318\end{array}$ & $\begin{array}{l}-0.13 \\
-0.18 \\
-0.12 \\
-0.09 \\
-0.04 \\
-0.10 \\
-0.08 \\
-0.07 \\
-0.06 \\
-0.05 \\
-0.08 \\
-0.12 \\
-0.03 \\
-0.09 \\
-0.10 \\
-0.04 \\
-0.03 \\
-0.07 \\
-0.12 \\
-0.07 \\
-0.03 \\
-0.12 \\
-0.08 \\
-0.12 \\
-0.09 \\
-0.06 \\
-0.14 \\
-0.08 \\
-0.03 \\
-0.03 \\
-0.07 \\
-0.05 \\
-0.06 \\
-0.04 \\
-0.12 \\
-0.11 \\
-0.02 \\
-0.13 \\
-0.15 \\
-0.10 \\
-0.11 \\
-0.03 \\
-0.05\end{array}$ & 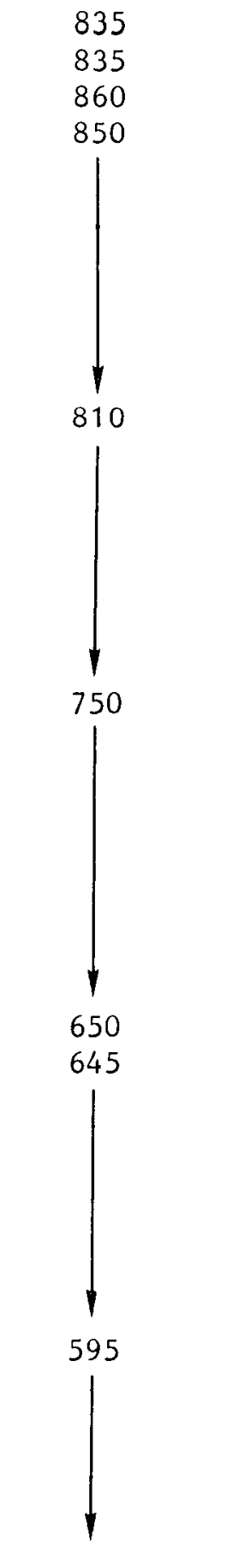 & i. & \\
\hline
\end{tabular}


TABLE 4B (Continued)

\begin{tabular}{|c|c|c|c|c|}
\hline $\begin{array}{c}\text { Record } \\
\text { No. }\end{array}$ & $\begin{array}{c}\text { Dimensional } \\
\text { Change } \\
\ln 1\left(+\frac{\Delta L}{L_{o}}\right) \\
(\%)\end{array}$ & $\begin{array}{l}\text { Average } \\
\text { Irradiation } \\
\text { Temperature } \\
\quad\left({ }^{\circ} \mathrm{C}\right)\end{array}$ & $\begin{array}{c}\text { Total } \\
\text { Fluence } \times 10^{-21} \\
\left(\mathrm{n} / \mathrm{cm}^{2}\right)(\mathrm{E}>0.18 \mathrm{MeV})\end{array}$ & $\begin{array}{c}\text { Previous } \\
\text { Record } \\
\text { No. }\end{array}$ \\
\hline $\begin{array}{l}6319 \\
6324 \\
6325 \\
6328 \\
6329 \\
5539 \\
5541 \\
5562 \\
5570 \\
5572 \\
5613 \\
5615 \\
5617 \\
5619 \\
5656 \\
5657 \\
5658 \\
5663 \\
5664 \\
5667 \\
5668 \\
5942 \\
5943 \\
5944 \\
5945 \\
5946 \\
5998 \\
5999 \\
6000 \\
6001 \\
6002 \\
6014 \\
6015 \\
6016 \\
6017 \\
6268 \\
6270 \\
6272\end{array}$ & $\begin{array}{l}-0.14 \\
-0.06 \\
-0.04 \\
-0.15 \\
-0.06 \\
-1.16 \\
-1.01 \\
-1.47 \\
-1.40 \\
-1.25 \\
-2.19 \\
-2.12 \\
-2.33 \\
-2.14 \\
-2.38 \\
-2.23 \\
-2.23 \\
-2.25 \\
-2.28 \\
-2.15 \\
-2.37 \\
-0.31 \\
-0.30 \\
-0.22 \\
-0.28 \\
-0.33 \\
-0.37 \\
-0.38 \\
-0.48 \\
-0.33 \\
-0.37 \\
-0.37 \\
-0.40 \\
-0.41 \\
-0.26 \\
-0.24 \\
-0.15 \\
-0.23\end{array}$ & 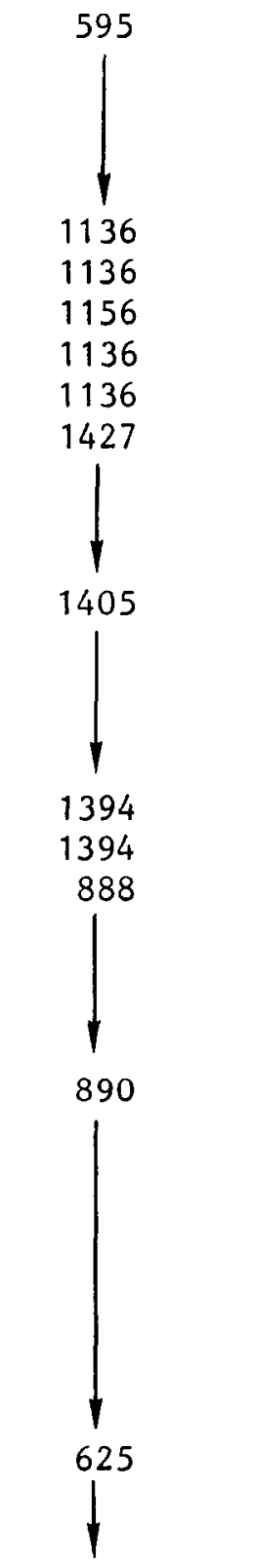 & 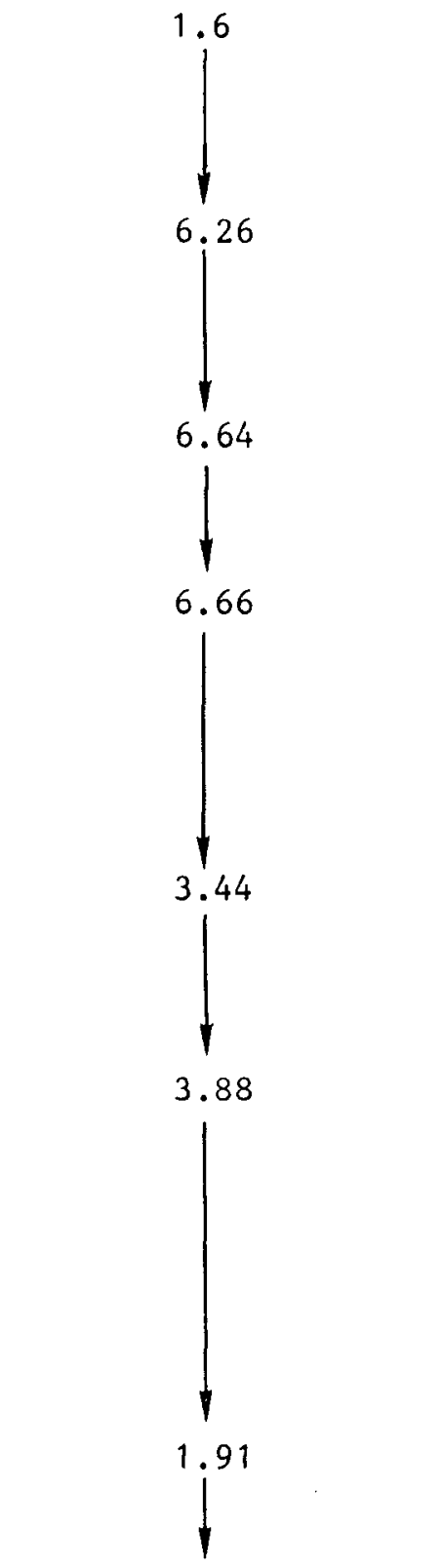 & $\begin{array}{l}2436 \\
2428 \\
2748 \\
2442 \\
2430 \\
2823 \\
2804 \\
2807 \\
2800 \\
2858 \\
2849 \\
2875 \\
2864 \\
2877 \\
2861 \\
2855 \\
2598 \\
2580 \\
2601 \\
2564 \\
2608 \\
2665 \\
2663 \\
2672 \\
2655 \\
2681 \\
2662 \\
2652 \\
2645 \\
2687 \\
2497 \\
2524 \\
2488\end{array}$ \\
\hline
\end{tabular}




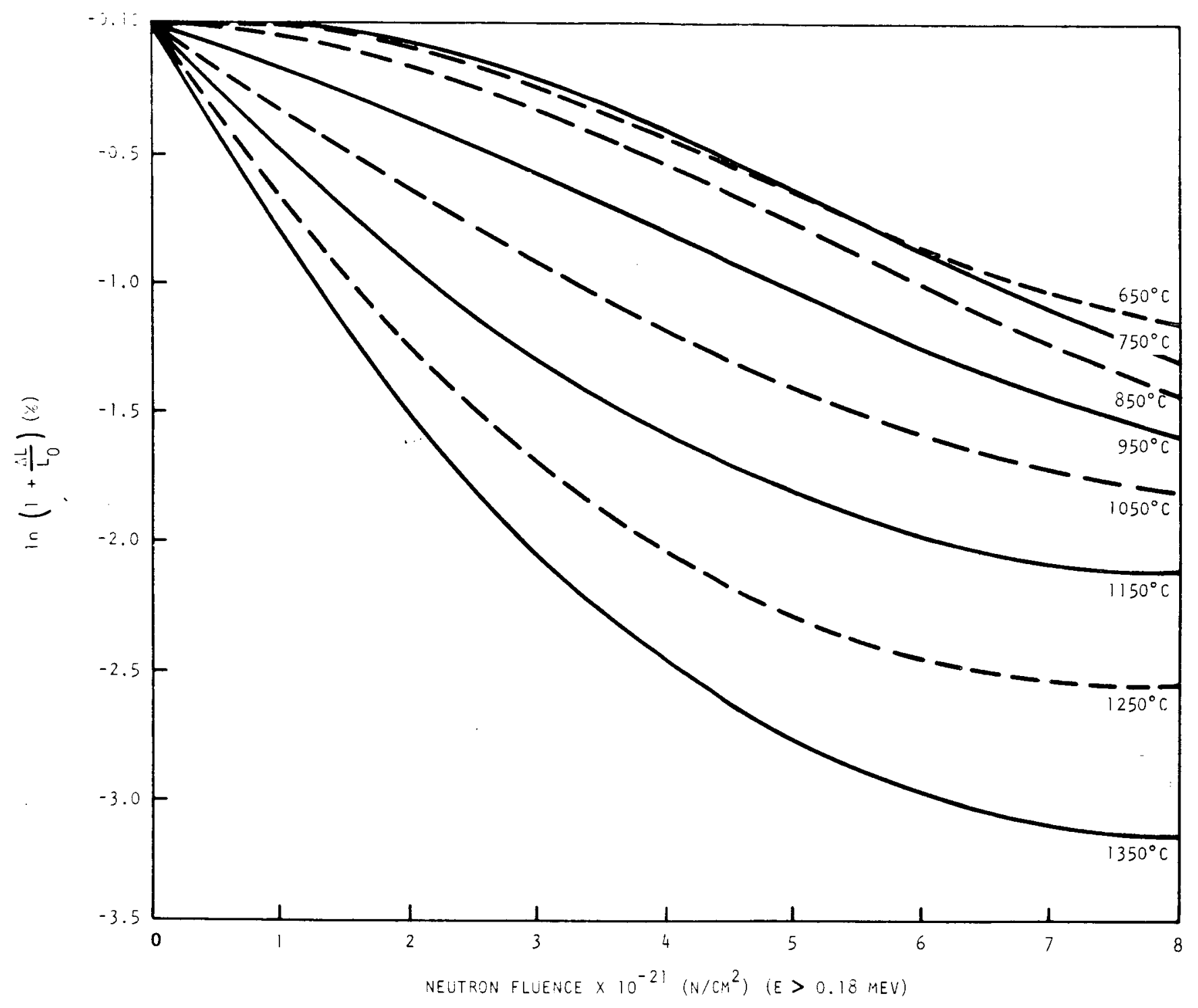

Fig. 4. Irradiation-induced dimensional changes in near-isotropic graphite; parallel (axial) orientation 


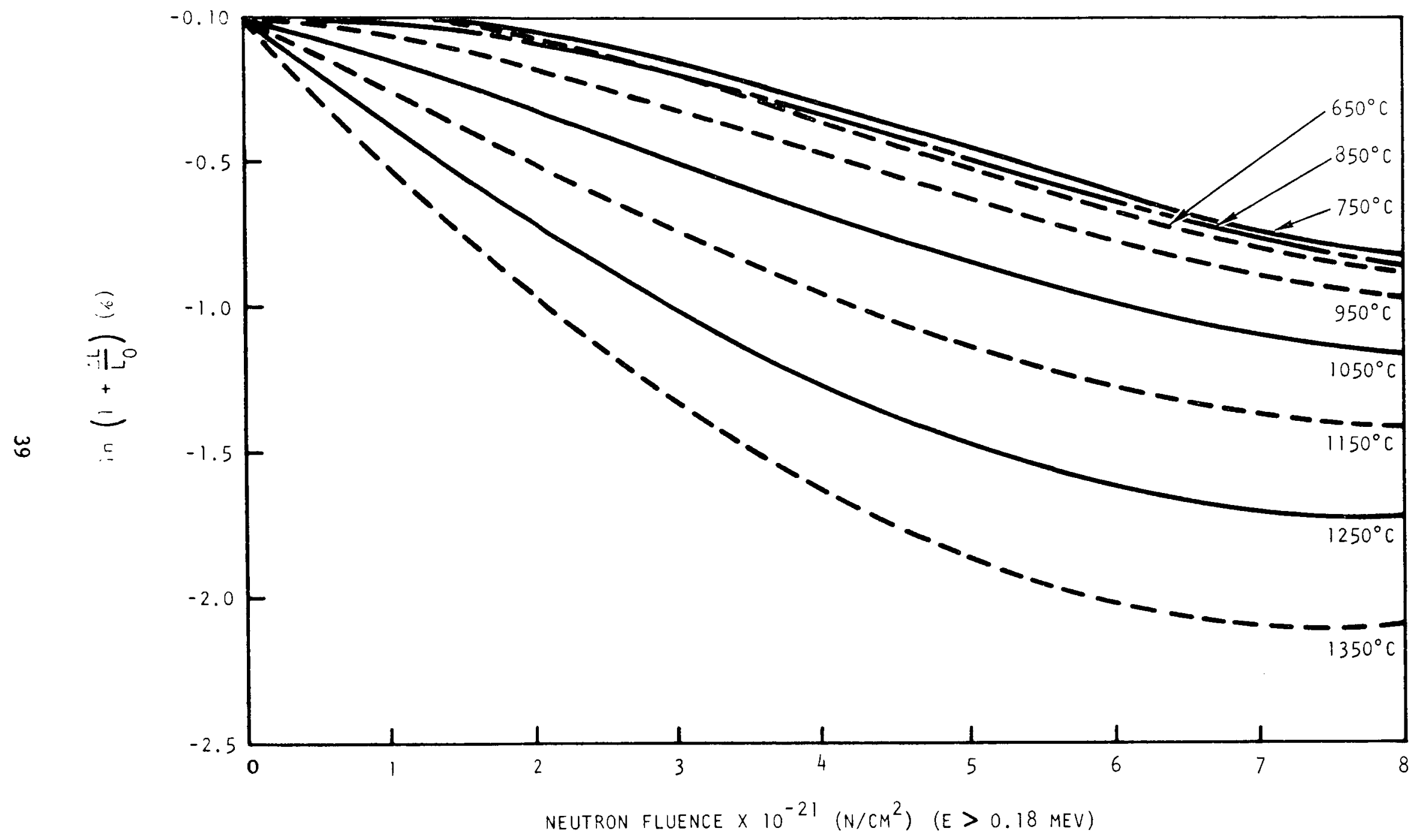

Fig. 5. Irradiation-induced dimensional changes in near-isotropic graphite; perpendicular (radial) orientation 
Ninety samples of $\mathrm{P}_{3}$ JHAN, a near-isotropic, pitch-coke graphite manufactured by Pechiney, were irradiated in OG-1 for comparison with the petroleum-coke near-isotropic graphites. As indicated by the data in Table 5, the $\mathrm{P}_{3}$ JHAN exhibited considerably more dimensional change under irradiation than the $H-451$ graphite. $P_{3}$ JHAN was also more anisotropic in its behavior.

\subsection{DIMENSIONAL CHANGE IN NEEDLE-COKE GRAPHITES}

The OG-1 experiment contained 195 parallel and 123 perpendicular specimens of H-327 graphite, 66 of which had been previously irradiated. The dimensional change data are given in Table 6 . For the most part, the H-327 was more dimensionally stable than had been observed in previous irradiations. As in the $\mathrm{H}-451$ data, no systematic variation of dimensional change with sample size was evident.

\subsection{MISCELLANEOUS GRAPHITES}

The dimensional changes observed in other graphites irradiated in OG-1 are described in Table 7. The H-453 underwent dimensional changes similar to other Great Lakes Carbon Company near-isotropic graphites. The 2020 graphite exhibited moderate dimensional changes whose orientation dependence was typical of molded materials.

The H-451C material, which was subjected to infiltration of fuel-rod binder pitch during cure-in-place treatment, exhibited the same dimensional change as the standard material $(-0.67 \%$ mean versus $-0.62 \%)$ after irradiation at $960^{\circ} \mathrm{C}$ to $3.4 \times 10^{21} \mathrm{n} / \mathrm{cm}^{2}$. Several H-451 samples $(\mathrm{IH}-451$, record numbers 6461-6465) were char-loaded by impregnation with oligofurane and heat-treated to $1100^{\circ} \mathrm{C}$, then irradiated together with standard $\mathrm{H}-451$ companion samples (record numbers 6466-6470). The char-loaded specimens contracted an average of $0.58 \%$, compared with $0.69 \%$ for the companion samples of standard H-451. Other specimens of standard H-451 contracted an average of $0.62 \%$ under the same conditions. 
TABLE 5

DIMENSIONAL CHANGE OF $\mathrm{P}_{3}$ JHAN

\begin{tabular}{|c|c|c|c|}
\hline $\begin{array}{c}\text { Record } \\
\text { No. }\end{array}$ & $\begin{array}{c}\text { Dimensional } \\
\text { Change } \\
\ln \left(1+\frac{\Delta L}{L_{o}}\right) \\
(\%)\end{array}$ & $\begin{array}{l}\quad \text { Average } \\
\text { Irradiation } \\
\text { Temperature } \\
\quad\left({ }^{\circ} \mathrm{C}\right)\end{array}$ & $\begin{array}{c}\text { Total } \\
\text { Fluence } \times 10^{-21} \\
(\mathrm{n} / \mathrm{cm})(\mathrm{E}>0.18 \mathrm{MeV})\end{array}$ \\
\hline
\end{tabular}

Parallel Orientation

\begin{tabular}{|c|c|c|c|}
\hline $\begin{array}{l}5319 \\
5321 \\
5323 \\
5325 \\
5327 \\
5329 \\
5331 \\
5333 \\
5335 \\
5676 \\
5678 \\
5684 \\
5686 \\
5734 \\
5736 \\
5740 \\
5742 \\
5744 \\
5746 \\
5750 \\
5752 \\
5754 \\
5756 \\
5760 \\
5762 \\
6164 \\
6165 \\
6170 \\
6171 \\
6174 \\
6175 \\
6180 \\
6181 \\
6184 \\
6185 \\
6190 \\
6191 \\
6332 \\
6334 \\
6336 \\
6338\end{array}$ & $\begin{array}{l}-1.53 \\
-2.04 \\
-2.02 \\
-1.48 \\
-1.99 \\
-2.00 \\
-1.44 \\
-1.83 \\
-2.11 \\
-1 \\
-3.96 \\
-4.00 \\
-4\end{array}$ & $\begin{array}{c}\mid \\
1 \\
1325 \\
1020 \\
\mid\end{array}$ & $\left.\right|^{1}$ \\
\hline & & 41 & \\
\hline
\end{tabular}


TABLE 5 (Continued)

\begin{tabular}{|c|c|c|c|}
\hline $\begin{array}{c}\text { Record } \\
\text { No. }\end{array}$ & $\begin{array}{c}\text { Dimensional } \\
\text { Change } \\
\ln \left(\begin{array}{c}1+\frac{\Delta \mathrm{L}}{\mathrm{L}_{\mathrm{o}}} \\
(\%)\end{array}\right. \\
\end{array}$ & $\begin{array}{l}\text { Average } \\
\text { Irradiation } \\
\text { Temperature } \\
\left({ }^{\circ} \mathrm{C}\right) \\
\end{array}$ & $\begin{array}{l}\text { Total } \\
\text { Fluence } \times 10^{-21} \\
(\mathrm{n} / \mathrm{cm})(\mathrm{E}>0.18 \mathrm{MeV})\end{array}$ \\
\hline \multicolumn{4}{|c|}{ Parallel Orientation } \\
\hline $\begin{array}{l}6342 \\
6344 \\
6347 \\
6349\end{array}$ & $\begin{array}{l}-0.20 \\
-0.22 \\
-0.17 \\
-0.21\end{array}$ & $\downarrow^{595}$ & $i^{1.6}$ \\
\hline \multicolumn{4}{|c|}{ Perpendicular } \\
\hline $\begin{array}{l}5320 \\
5322 \\
5324 \\
5326 \\
5328 \\
5330 \\
5332 \\
5334 \\
5336 \\
5677 \\
5679 \\
5685 \\
5687 \\
5735 \\
5737 \\
5741 \\
5743 \\
5745 \\
5747 \\
5751 \\
5753 \\
5757 \\
5761 \\
5763 \\
6166 \\
6167 \\
6172 \\
6173 \\
6176 \\
6177 \\
6182 \\
6183 \\
6186 \\
6187 \\
6192 \\
6193\end{array}$ & $\begin{array}{l}-0.69 \\
-0.76 \\
-0.58 \\
-0.73 \\
-0.87 \\
-0.83 \\
-0.66 \\
-0.78 \\
-0.73 \\
-1.66 \\
-1.44 \\
-1.65 \\
-1.68 \\
-0.81 \\
-0.90 \\
-0.87 \\
-0.84 \\
-0.81 \\
-0.86 \\
-0.71 \\
-0.67 \\
-0.70 \\
-0.81 \\
-0.83 \\
-0.11 \\
-0.08 \\
-0.17 \\
-0\end{array}$ & $\mid$ & $\left.\right|^{3.4}$ \\
\hline
\end{tabular}


TABLE 5 (Continued)

\begin{tabular}{|c|c|c|c|}
\hline $\begin{array}{c}\text { Record } \\
\text { No. }\end{array}$ & $\begin{array}{c}\text { Dimensional } \\
\text { Change } \\
\ln \left(1+\frac{\Delta \mathrm{L}}{\mathrm{L}_{\mathrm{o}}}\right)\end{array}$ & $\begin{array}{l}\text { Average } \\
\text { Irradiation } \\
\text { Temperature } \\
\quad\left({ }^{\circ} \mathrm{C}\right)\end{array}$ & $\begin{array}{c}\text { Total } \\
\text { Fluence } \times 10^{-21} \\
(\mathrm{n} / \mathrm{cm})(\mathrm{E}>0.18 \mathrm{MeV})\end{array}$ \\
\hline \multicolumn{4}{|c|}{ Perpendicular } \\
\hline $\begin{array}{l}6333 \\
6335 \\
6337 \\
6339 \\
6343 \\
6345 \\
6348 \\
6350\end{array}$ & $\begin{array}{l}-0.15 \\
-0.10 \\
-0.17 \\
-0.06 \\
-0.18 \\
-0.06 \\
-0.15 \\
-0.13\end{array}$ & $\left.\right|^{595}$ & $i^{1.6}$ \\
\hline
\end{tabular}


TABLE 6A

DIMENSIONAL CHANGES OF NEEDLE-COKE H-327 GRAPHITE

FROM CAPSULE OG-1

PARALLEL ORIENTATION

\begin{tabular}{|c|c|c|c|c|}
\hline $\begin{array}{c}\text { Record } \\
\text { No. }\end{array}$ & $\begin{array}{l}\text { Dimensional } \\
\text { Change } \\
\ln \left(1+\frac{\Delta \mathrm{L}}{\mathrm{L}_{0}}\right)\end{array}$ & $\begin{array}{c}\text { Total } \\
\text { Fluence } \times 10^{-21} \\
\left(\mathrm{n} / \mathrm{cm}^{2}\right)(\mathrm{E}>0.18 \mathrm{MeV})\end{array}$ & $\begin{array}{c}\text { Average } \\
\text { Irradiation } \\
\text { Temperature } \\
\quad\left({ }^{\circ} \mathrm{C}\right)\end{array}$ & $\begin{array}{l}\text { Previous } \\
\text { Record } \\
\text { No. }\end{array}$ \\
\hline $\begin{array}{l}5005 \\
5009 \\
5013 \\
5017 \\
5021 \\
5025 \\
5029 \\
5033 \\
5037 \\
5041 \\
5114 \\
5115 \\
5118 \\
5119 \\
5124 \\
5125 \\
5128 \\
5129 \\
5134 \\
5135 \\
5147 \\
5151 \\
5155 \\
5159 \\
5163 \\
5167 \\
5171 \\
5175 \\
5179 \\
5182 \\
5185 \\
5186 \\
5195 \\
5196 \\
5205 \\
5215 \\
5216 \\
5241 \\
5242 \\
5251\end{array}$ & $\begin{array}{l}-0.15 \\
-0.16 \\
-0.17 \\
-0.14 \\
-0.11 \\
-0.13 \\
-0.11 \\
-0.15 \\
-0.20 \\
-0.18 \\
-0.14 \\
-0.16 \\
-0.16 \\
-0.20 \\
-0.15 \\
-0.21 \\
-0.14 \\
-0.18 \\
-0.14 \\
-0.20 \\
-0 \\
-0.25 \\
-0.24 \\
-0.17 \\
-0.24 \\
-0 \\
-0.26 \\
-0.26 \\
-0\end{array}$ & 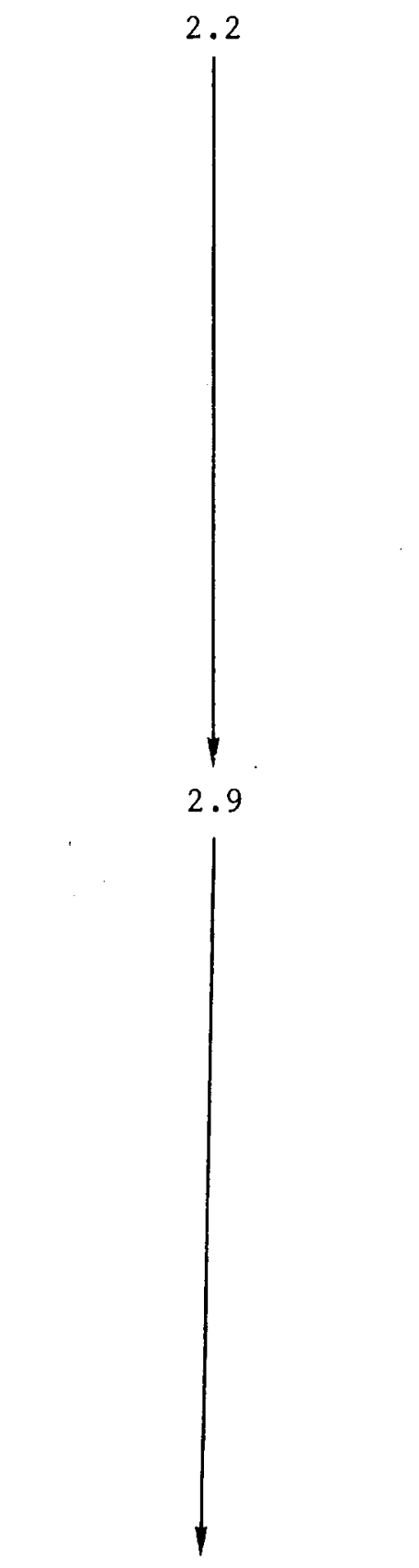 & $\mid$ & \\
\hline
\end{tabular}


TABLE 6A (Continued)

\begin{tabular}{|c|c|c|c|c|}
\hline $\begin{array}{c}\text { Record } \\
\text { No. }\end{array}$ & $\begin{array}{l}\text { Dimensional } \\
\text { Change } \\
\ln \left(1+\frac{\Delta \mathrm{L}}{\mathrm{L}_{0}}\right)\end{array}$ & $\begin{array}{c}\text { Total } \\
\text { Fluence } \times 10^{-21} \\
\left(\mathrm{n} / \mathrm{cm}^{2}\right)(\mathrm{E}>0.18 \mathrm{MeV})\end{array}$ & $\begin{array}{c}\text { Average } \\
\text { Irradiation } \\
\text { Temperature } \\
\left({ }^{\circ} \mathrm{C}\right)\end{array}$ & $\begin{array}{l}\text { Previous } \\
\text { Record } \\
\text { No. }\end{array}$ \\
\hline $\begin{array}{l}5252 \\
5257 \\
5258 \\
5261 \\
5262 \\
5263 \\
5264 \\
5267 \\
5268 \\
5271 \\
5272 \\
5273 \\
5274 \\
5277 \\
5278 \\
5281 \\
5282 \\
5291 \\
5292 \\
5466 \\
5467 \\
5473 \\
5474 \\
5477 \\
5478 \\
5509 \\
5510 \\
5511 \\
5513 \\
5514 \\
5523 \\
5524 \\
5533 \\
5534 \\
5543 \\
5544 \\
5546 \\
5547 \\
5550 \\
5551 \\
5555 \\
5556 \\
5557\end{array}$ & $\begin{array}{l}-0.31 \\
-0.24 \\
-0.34 \\
-0.32 \\
-0.27 \\
-0.34 \\
-0.33 \\
-0.25 \\
-0.21 \\
-0.29 \\
-0.27 \\
-0 \\
-0.21 \\
-0.33 \\
-0.31 \\
-0.28 \\
-0 \\
-0.20 \\
-0.28 \\
-0.33 \\
-0.28 \\
-0 \\
-0.62 \\
-0.50 \\
-0\end{array}$ & $\left.\right|^{j^{2.9}}$ & 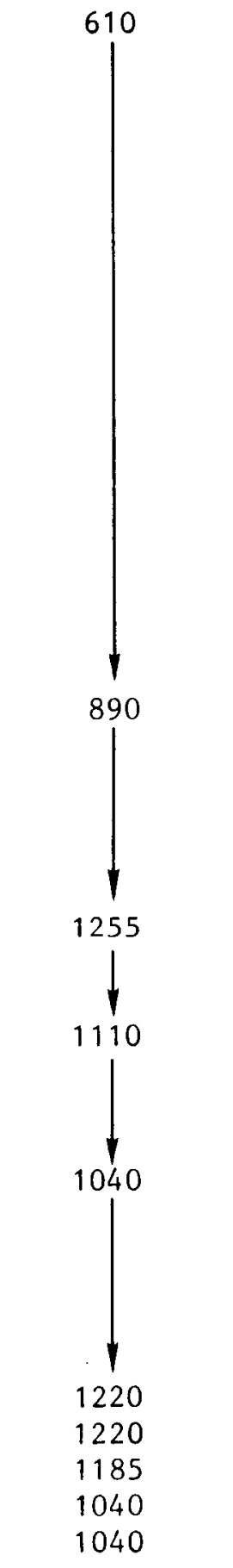 & $\begin{array}{l}2433 \\
2447 \\
2774\end{array}$ \\
\hline
\end{tabular}


TABLE 6A (Continued)

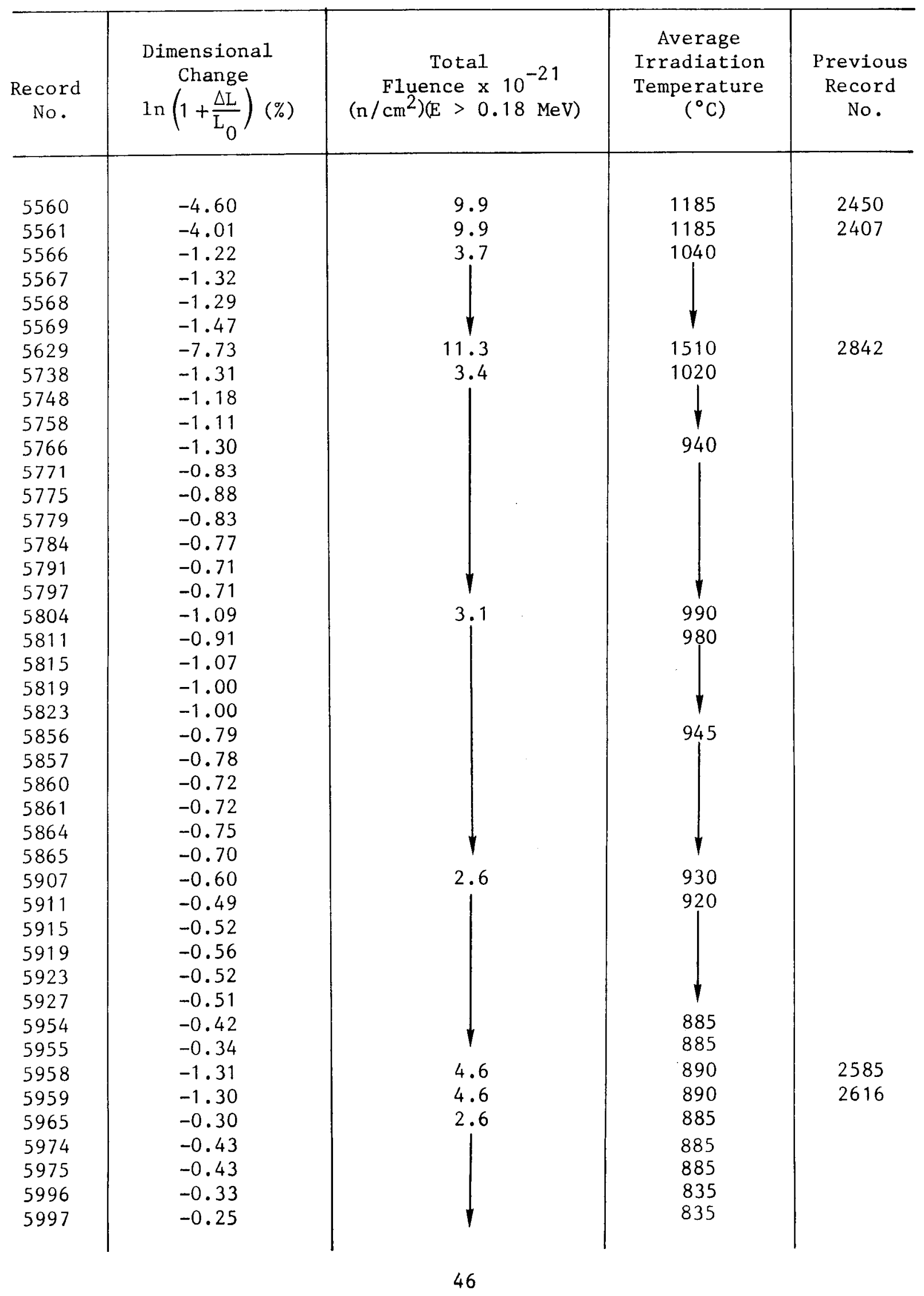


TABLE 6A (Continued)

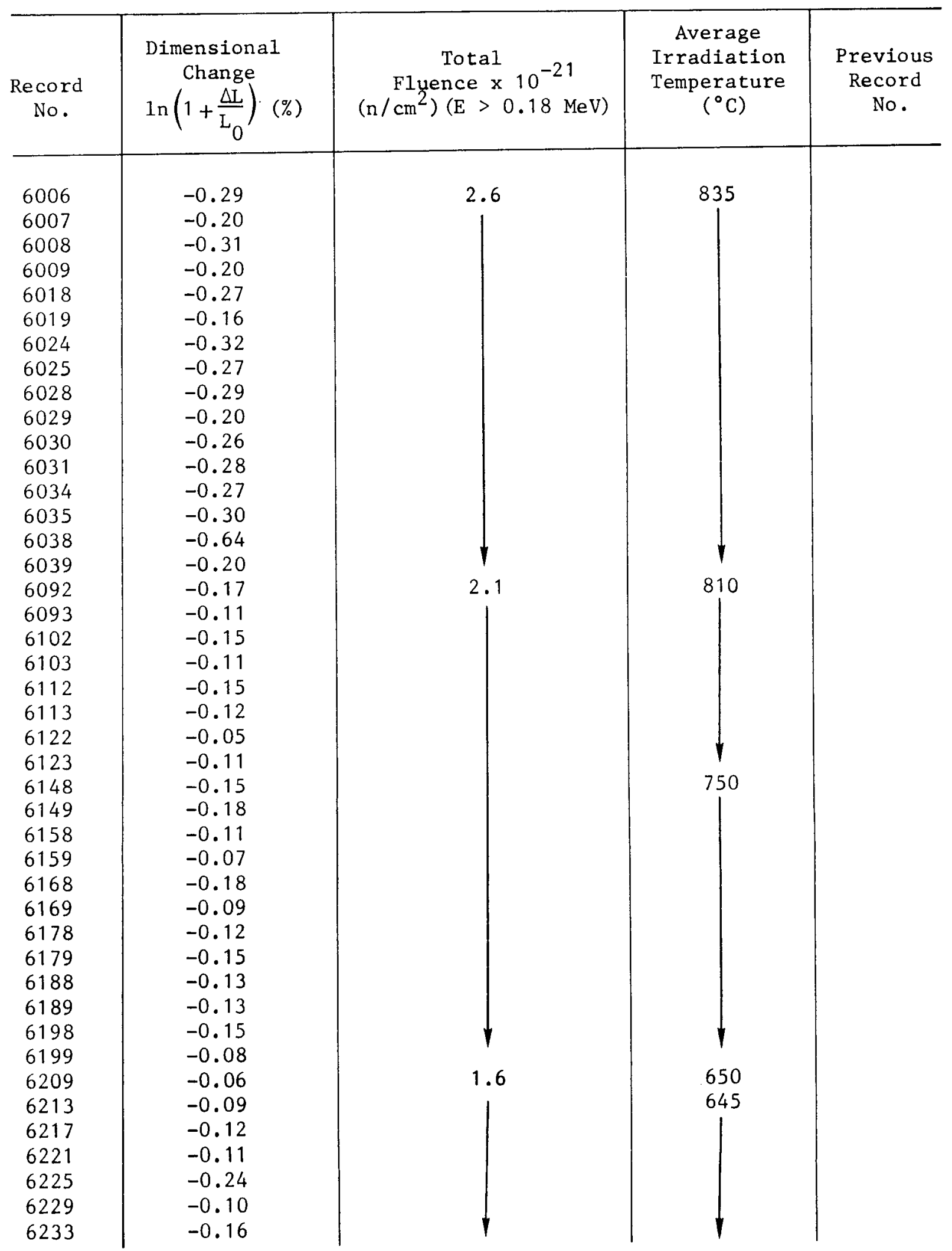

47 
TABLE 6A (Continued)

\begin{tabular}{|c|c|c|c|c|}
\hline $\begin{array}{c}\text { Record } \\
\text { No. }\end{array}$ & $\begin{array}{l}\text { Dimensional } \\
\text { Change } \\
\ln \left(1+\frac{\Delta \mathrm{L}}{\mathrm{L}}\right)(\%)\end{array}$ & $\begin{array}{c}\text { Total } \\
\text { Fluence } \times 10^{-21} \\
\left(\mathrm{n} / \mathrm{cm}^{2}\right)(\mathrm{E}>0.18 \mathrm{MeV})\end{array}$ & $\begin{array}{c}\text { Average } \\
\text { Irradiation } \\
\text { Temperature } \\
\left({ }^{\circ} \mathrm{C}\right)\end{array}$ & $\begin{array}{l}\text { Previous } \\
\text { Record } \\
\text { No. }\end{array}$ \\
\hline $\begin{array}{l}6237 \\
6241 \\
6246 \\
6250 \\
6251 \\
6254 \\
6255 \\
6260 \\
6261 \\
6264 \\
6265 \\
6301\end{array}$ & $\begin{array}{l}-0.15 \\
-0.10 \\
-0.41 \\
-0.34 \\
-0.20 \\
-0.37 \\
-0.25 \\
-0.40 \\
-0.19 \\
-0.43 \\
-0.34 \\
-0.15\end{array}$ & $\begin{array}{c}1.6 \\
1.6 \\
2.4 \\
\left.\right|_{1.6}\end{array}$ & $\begin{array}{l}645 \\
645 \\
625 \\
\mid \\
595\end{array}$ & $\begin{array}{l}2465 \\
2458 \\
2410 \\
2472 \\
2457 \\
2493 \\
2435 \\
2474 \\
2698\end{array}$ \\
\hline
\end{tabular}


TABLE 6B

DIMENSIONAL CHANGES OF NEEDLE-COKE H-327 GRAPHITE

FROM CAPSULE OG-1

PERPENDICULAR ORIENTATION

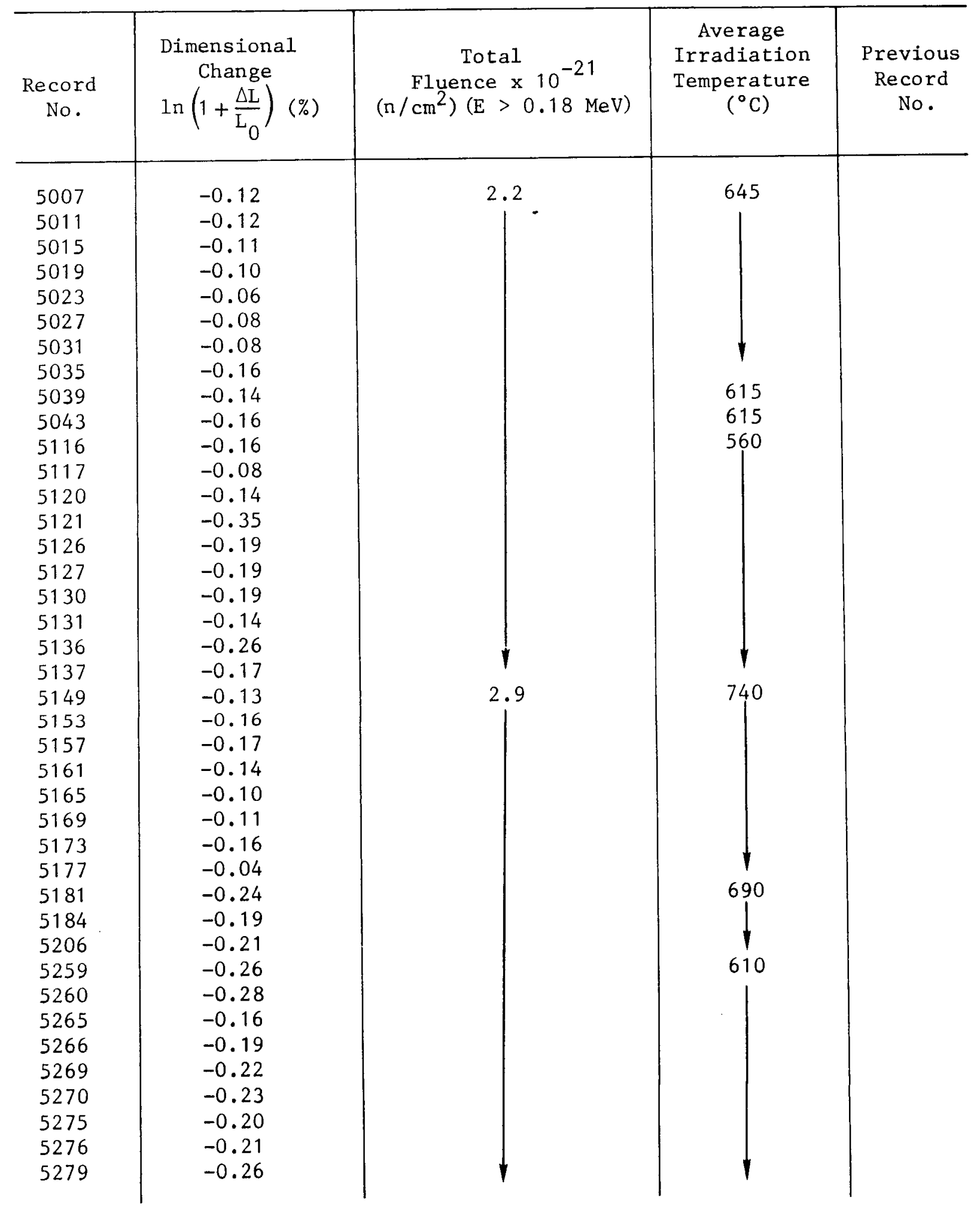


TABLE 6B (Continued)

\begin{tabular}{|c|c|c|c|c|}
\hline $\begin{array}{c}\text { Record } \\
\text { No. }\end{array}$ & $\begin{array}{l}\text { Dimensional } \\
\text { Change } \\
\ln \left(1+\frac{\Delta \mathrm{L}}{\mathrm{L}_{0}}\right)(\%)\end{array}$ & $\begin{array}{c}\text { Total } \\
\text { Fluence } \times 10^{-21} \\
\left(\mathrm{n} / \mathrm{cm}^{2}\right)(\mathrm{E}>0.18 \mathrm{MeV})\end{array}$ & $\begin{array}{l}\text { Irradiation } \\
\text { Temperature } \\
\left({ }^{\circ} \mathrm{C}\right)\end{array}$ & $\begin{array}{l}\text { Previous } \\
\text { Record } \\
\text { No. }\end{array}$ \\
\hline $\begin{array}{l}5280 \\
5357 \\
5358 \\
5362 \\
5363 \\
5369 \\
5370 \\
5506 \\
5507 \\
5548 \\
5549 \\
5552 \\
5553 \\
5558 \\
5559 \\
5630 \\
5739 \\
5749 \\
5759 \\
5767 \\
5772 \\
5776 \\
5780 \\
5785 \\
5792 \\
5798 \\
5809 \\
5813 \\
5817 \\
5821 \\
5825 \\
5858 \\
5859 \\
5862 \\
5863 \\
5866 \\
5867 \\
5909 \\
5913 \\
5917 \\
5921 \\
5925 \\
5929\end{array}$ & $\begin{array}{r}-0.26 \\
-0.43 \\
-0.36 \\
-0.48 \\
-0.42 \\
-0.65 \\
-0.40 \\
9.43 \\
-0.70 \\
-0.06 \\
0.91 \\
-0.25 \\
0.90 \\
-0.04 \\
0.35 \\
2.39 \\
-0.32 \\
-0.51 \\
-0.50 \\
-0.52 \\
-0.36 \\
-0.40 \\
-0.42 \\
-0.41 \\
-0.32 \\
-0.40 \\
-0\end{array}$ & 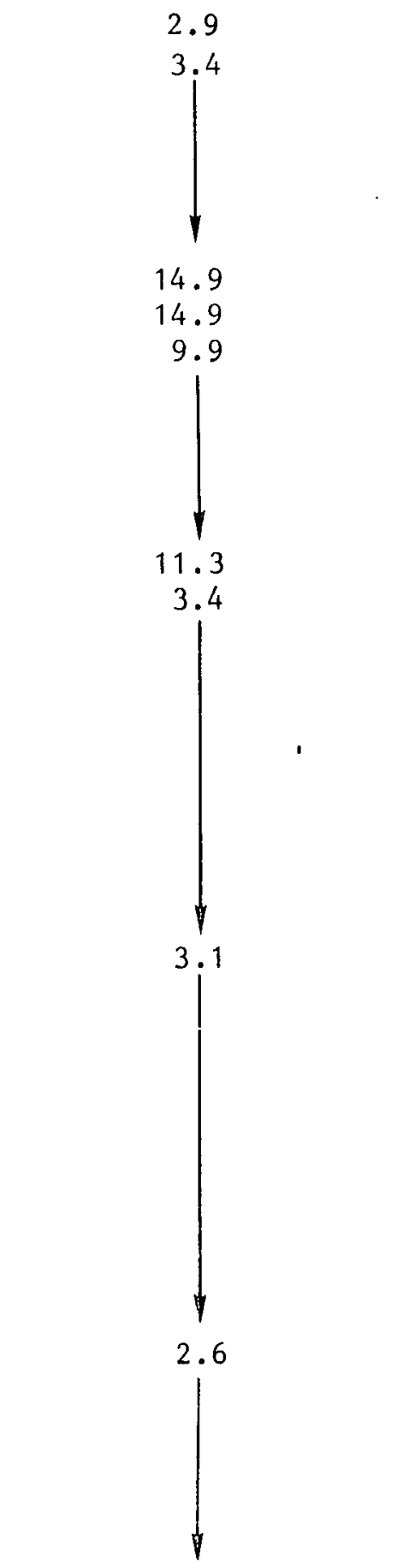 & 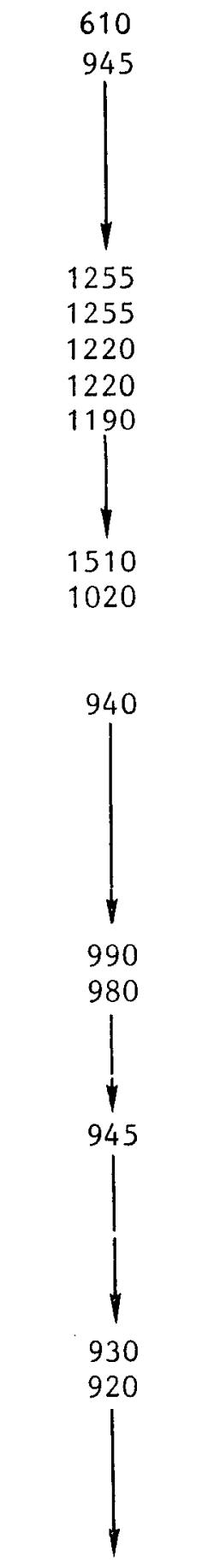 & $\begin{array}{l}2910 \\
2909 \\
2443 \\
2455 \\
2772 \\
2760 \\
2451 \\
2429 \\
2839\end{array}$ \\
\hline
\end{tabular}


TABLE 6B (Continued)

\begin{tabular}{|c|c|c|c|c|}
\hline $\begin{array}{c}\text { Record } \\
\text { No. }\end{array}$ & $\begin{array}{l}\text { Dimensional } \\
\text { Change } \\
\ln \left(1+\frac{\Delta \mathrm{L}}{\mathrm{L}_{0}}\right)(\%)\end{array}$ & $\begin{array}{c}\text { Total } \\
\text { Fluence } \times 10^{-21} \\
\left(\mathrm{n} / \mathrm{cm}^{2}\right)(\mathrm{E}>0.18 \mathrm{MeV})\end{array}$ & $\begin{array}{c}\text { Average } \\
\text { Irradiation } \\
\text { Temperature } \\
\left({ }^{\circ} \mathrm{C}\right)\end{array}$ & $\begin{array}{l}\text { Previous } \\
\text { Record } \\
\text { No. }\end{array}$ \\
\hline $\begin{array}{l}5956 \\
5957 \\
6026 \\
6027 \\
6032 \\
6033 \\
6036 \\
6037 \\
6211 \\
6215 \\
6219 \\
6223 \\
6227 \\
6231 \\
6235 \\
6239 \\
6243 \\
6244 \\
6245 \\
6248 \\
6249 \\
6252 \\
6253 \\
6258 \\
6259 \\
6262 \\
6263 \\
6303\end{array}$ & $\begin{array}{l}-0.79 \\
-0.59 \\
-0.19 \\
-0.12 \\
-0.23 \\
-0.02 \\
-0.14 \\
-0.08 \\
-0.06 \\
-0.12 \\
-0.07 \\
-0.04 \\
-0.06 \\
-0.04 \\
-0.09 \\
-0.05 \\
-0.06 \\
-0.54 \\
-0.31 \\
-0.39 \\
-0.33 \\
-0.46 \\
-0.33 \\
-0.46 \\
-0.25 \\
-0.43\end{array}$ & $\left.\right|^{4.6}$ & $\left.\right|^{890}$ & $\begin{array}{l}2638 \\
2552\end{array}$ \\
\hline
\end{tabular}


TABLE 7

DIMENSIONAL CHANGES IN OG-1 GRAPHITES

\begin{tabular}{|c|c|c|c|c|c|c|}
\hline Grade & Orientation & $\begin{array}{l}\text { Total Fluence } \\
\times 10^{-21}\left(\mathrm{n} / \mathrm{cm}^{2}\right) \\
(E>0.18 \mathrm{MeV})\end{array}$ & $\begin{array}{c}\text { Average } \\
\text { Irradiation } \\
\text { Temperature } \\
\left({ }^{\circ} \mathrm{C}\right)\end{array}$ & $\begin{array}{c}\text { Dimensional } \\
\text { Change } \\
\ln \left(1+\frac{\Delta \mathrm{L}}{\mathrm{L}_{\mathrm{o}}}\right) \\
(\%)\end{array}$ & $\begin{array}{c}\text { Record } \\
\text { No. }\end{array}$ & $\begin{array}{c}\text { Previous } \\
\text { Record } \\
\text { No. }\end{array}$ \\
\hline$H-453$ & Parallel & $i^{2.2}$ & 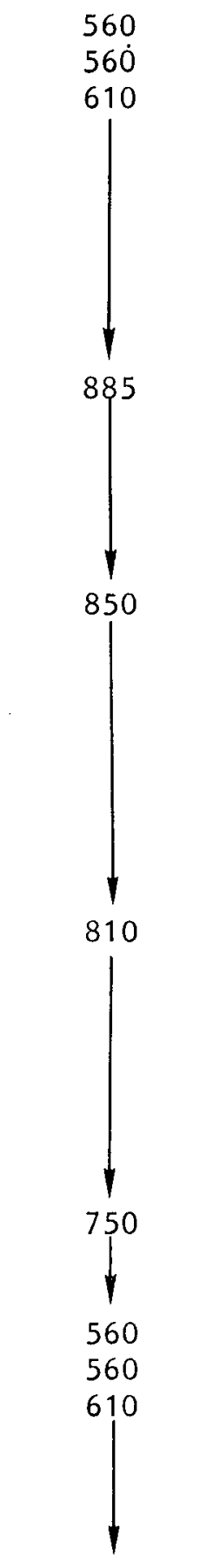 & $\begin{array}{l}-0.18 \\
-0.22 \\
-0.34 \\
-0.34 \\
-0.32 \\
-0.35 \\
-0.21 \\
-0.28 \\
-0.22 \\
-0.26 \\
-0.50 \\
-0.45 \\
-0.49 \\
-0.45 \\
-0.55 \\
-0.51 \\
-0.18 \\
-0.20 \\
-0.20 \\
-0.17 \\
-0.17 \\
-0.17 \\
-0.15 \\
-0.20 \\
-0.19 \\
-0.15 \\
-0.14 \\
-0.15 \\
-0.17 \\
-0.16 \\
-0.14 \\
-0.15 \\
-0.17 \\
-0.14 \\
-0.09 \\
-0.23 \\
-0.18 \\
-0.19 \\
-0.27 \\
-0.17 \\
-0.34 \\
-0.18\end{array}$ & $\begin{array}{l}5138 \\
5139 \\
5283 \\
5284 \\
5287 \\
5288 \\
5293 \\
5294 \\
5297 \\
5298 \\
5881 \\
5882 \\
5885 \\
5886 \\
5893 \\
5894 \\
6049 \\
6053 \\
6057 \\
6061 \\
6065 \\
6069 \\
6073 \\
6077 \\
6081 \\
6085 \\
6089 \\
6095 \\
6099 \\
6105 \\
6109 \\
6115 \\
6119 \\
6151 \\
6155 \\
6161 \\
5140 \\
5141 \\
5285 \\
5286 \\
5289 \\
5295\end{array}$ & \\
\hline
\end{tabular}


TABLE 7 (Continued)

\begin{tabular}{|c|c|c|c|c|c|c|}
\hline Grade & Orientation & $\begin{array}{l}\text { Total Fluence } \\
\times 10^{-21}\left(\mathrm{n} / \mathrm{cm}^{2}\right) \\
(\mathrm{E}>0.18 \mathrm{MeV})\end{array}$ & $\begin{array}{l}\text { Average } \\
\text { Irradiation } \\
\text { Temperature } \\
\quad\left({ }^{\circ} \mathrm{C}\right)\end{array}$ & $\begin{array}{c}\text { Dimensional } \\
\text { Change } \\
\ln \left(1+\frac{\Delta \mathrm{L}}{\mathrm{L}_{\circ}}\right) \\
(\%)\end{array}$ & $\begin{array}{c}\text { Record } \\
\text { No. }\end{array}$ & $\begin{array}{l}\text { Previous } \\
\text { Record } \\
\text { No. }\end{array}$ \\
\hline$H-453$ & Para11e1 & $i^{3} i^{9}$ & $\left.\right|^{805}$ & $\begin{array}{l}-0.15 \\
-0.16 \\
-0.19 \\
-0.24 \\
-0.10 \\
-0.23 \\
-0.20 \\
-0.26 \\
-0.07 \\
-0.09 \\
-0.10 \\
-0.02 \\
-0.04 \\
-0.09 \\
-0.06 \\
-0.05 \\
-0.07 \\
-0.04 \\
-0.06 \\
-0.06 \\
-0.02 \\
-0.14 \\
-0.07 \\
-0.08 \\
-0 \\
-0.08 \\
-0.04 \\
-0.07 \\
-0.02 \\
-0 \\
-0.10 \\
-0.37 \\
-0.45 \\
-0.35 \\
-0\end{array}$ & $\begin{array}{l}5296 \\
5299 \\
5300 \\
5883 \\
5884 \\
5887 \\
5888 \\
5895 \\
5896 \\
6051 \\
6055 \\
6059 \\
6063 \\
6067 \\
6071 \\
6075 \\
6079 \\
6083 \\
6087 \\
6091 \\
6097 \\
6101 \\
6107 \\
6111 \\
6117 \\
6121 \\
6153 \\
6157 \\
6163 \\
5070 \\
5072 \\
5074 \\
5076 \\
5078 \\
5080 \\
5082 \\
5084 \\
5086 \\
5088 \\
5217 \\
5219 \\
5221 \\
5223 \\
5225 \\
5227 \\
5229\end{array}$ & \\
\hline
\end{tabular}


TABLE 7 (Continued)

\begin{tabular}{|c|c|c|c|c|c|c|}
\hline Grade & Orientation & $\begin{array}{l}\text { Total Fluence } \\
\times 10^{-21}\left(\mathrm{n} / \mathrm{cm}^{2}\right) \\
(\mathrm{E}>0.18 \mathrm{MeV})\end{array}$ & $\begin{array}{c}\text { Average } \\
\text { Irradiation } \\
\text { Temperature } \\
\left({ }^{\circ} \mathrm{C}\right) \\
\end{array}$ & $\begin{array}{c}\text { Dimensional } \\
\text { Change } \\
\ln \left(1+\frac{\Delta \mathrm{L}}{\mathrm{L}_{\mathrm{o}}}\right) \\
(\%)\end{array}$ & $\begin{array}{c}\text { Record } \\
\text { No. }\end{array}$ & $\begin{array}{c}\text { Previous } \\
\text { Record } \\
\text { No. } \\
\end{array}$ \\
\hline 2020 & Paralle1 & $i^{3.9}$ & $\left.\right|_{915} ^{630}$ & 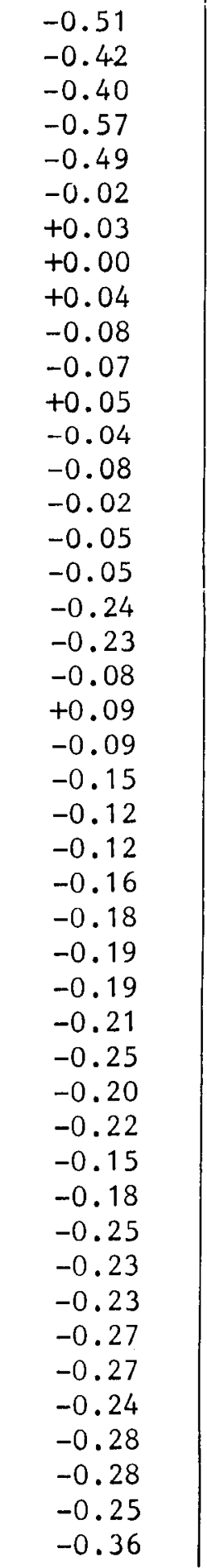 & $\begin{array}{l}5231 \\
5233 \\
5235 \\
5237 \\
5239 \\
5386 \\
5388 \\
5392 \\
5394 \\
5398 \\
5400 \\
5404 \\
5406 \\
5410 \\
5412 \\
5416 \\
5418 \\
5425 \\
5427 \\
5429 \\
5431 \\
5870 \\
5872 \\
5874 \\
5876 \\
5976 \\
5978 \\
5980 \\
5982 \\
5984 \\
5986 \\
5988 \\
5990 \\
5992 \\
5994 \\
6124 \\
6126 \\
6128 \\
6130 \\
6132 \\
6134 \\
6136 \\
6138 \\
6140 \\
6142\end{array}$ & \\
\hline
\end{tabular}


TABLE 7 (Continued)

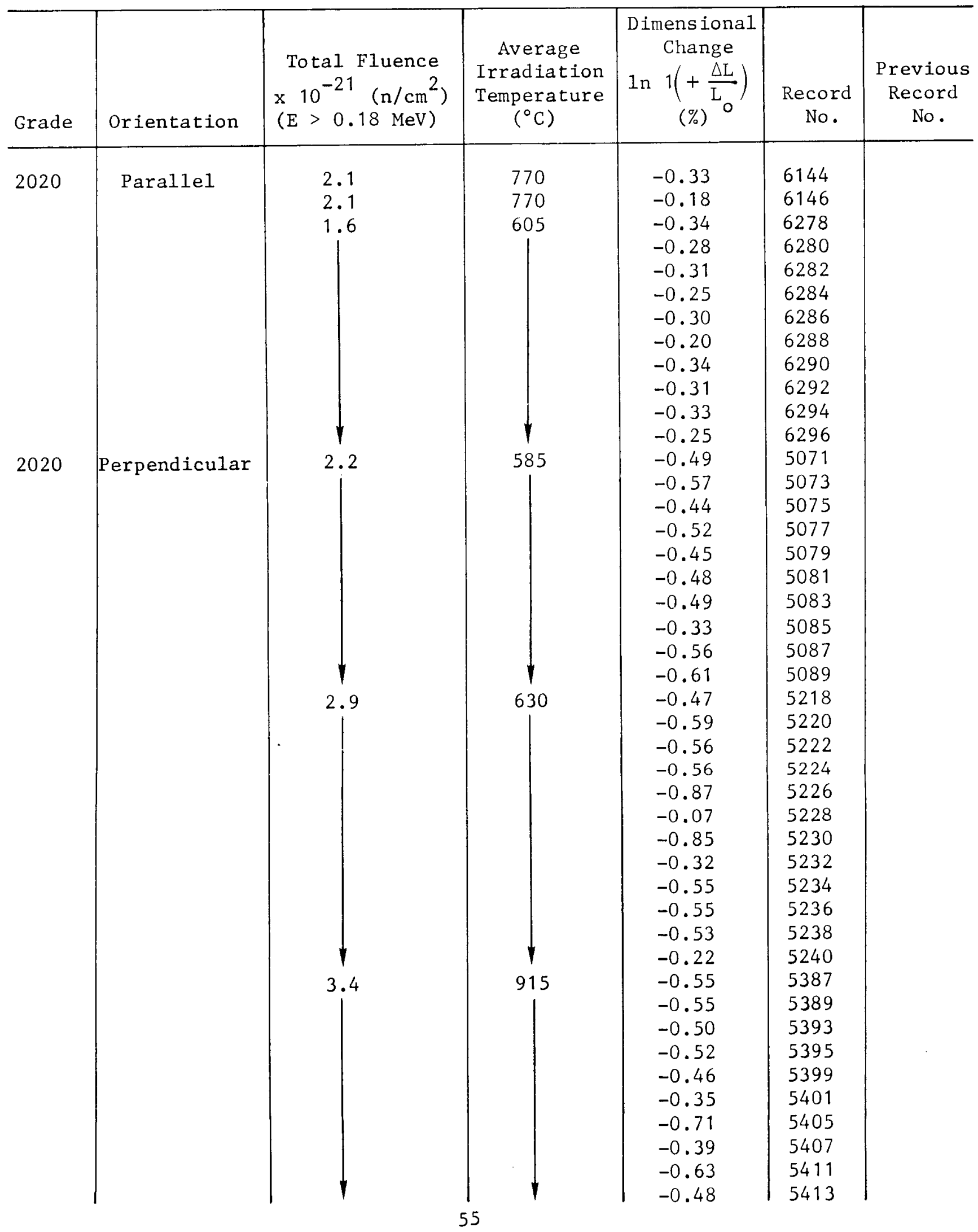


TABLE 7 (Continued)

\begin{tabular}{|c|c|c|c|c|c|c|}
\hline Grade & Orientation & $\begin{array}{l}\text { Total Fluence } \\
\times 10^{-21}\left(\mathrm{n} / \mathrm{cm}^{2}\right) \\
(\mathrm{E}>0.18 \mathrm{MeV})\end{array}$ & $\begin{array}{c}\text { Average } \\
\text { Irradiation } \\
\text { Temperature } \\
\left({ }^{\circ} \mathrm{C}\right)\end{array}$ & $\begin{array}{c}\text { Dimensional } \\
\text { Change } \\
\text { In } 1\left(+\frac{\Delta \mathrm{L}}{\mathrm{L}}\right) \\
(\%)\end{array}$ & $\begin{array}{c}\text { Record } \\
\text { No. }\end{array}$ & $\begin{array}{l}\text { Previous } \\
\text { Record } \\
\text { No. }\end{array}$ \\
\hline 2020 & Perpendicular & $i^{3.4}$ & $\mid$ & $\begin{array}{l}-0.48 \\
-0.58 \\
-0.93 \\
-0.57 \\
-0.81 \\
-0.76 \\
-0.66 \\
-0.44 \\
-0.50 \\
-0.36 \\
-0.51 \\
-0.38 \\
-0.50 \\
-0.29 \\
-0.43 \\
-0.31 \\
-0.46 \\
-0.36 \\
-0.55 \\
-0.36 \\
-0.40 \\
-0.35 \\
-0.40 \\
-0.23 \\
-0.50 \\
-0.26 \\
-0.33 \\
-0.25 \\
-0.49 \\
-0.44 \\
-0.20 \\
-0.36 \\
-0.62 \\
-0.28 \\
-0.46 \\
-0.24 \\
-0.36 \\
-0.29 \\
-0.39 \\
-0.23 \\
-0.35 \\
-0.23\end{array}$ & $\begin{array}{l}5417 \\
5419 \\
5526 \\
5528 \\
5530 \\
5532 \\
5871 \\
5873 \\
5875 \\
5877 \\
5977 \\
5979 \\
5981 \\
5983 \\
5985 \\
5987 \\
5989 \\
5991 \\
5993 \\
5995 \\
6125 \\
6127 \\
6129 \\
6131 \\
6133 \\
6135 \\
6137 \\
6139 \\
6141 \\
6143 \\
6145 \\
6147 \\
6279 \\
6281 \\
6283 \\
6285 \\
6287 \\
6289 \\
6291 \\
6293 \\
6295 \\
6297\end{array}$ & \\
\hline
\end{tabular}


TABLE 7 (Continued)

\begin{tabular}{|c|c|c|c|c|c|c|}
\hline Grade & Orientation & $\begin{array}{l}\text { Total Fluence } \\
\times 10^{-21}\left(\mathrm{n} / \mathrm{cm}^{2}\right) \\
(\mathrm{E}>0.18 \mathrm{MeV})\end{array}$ & $\begin{array}{c}\text { Average } \\
\text { Irradiation } \\
\text { Temperature } \\
\left({ }^{\circ} \mathrm{C}\right)\end{array}$ & $\begin{array}{c}\text { Dimensional } \\
\text { Change } \\
\ln \left(1+\frac{\Delta \mathrm{L}}{\mathrm{L}_{\mathrm{o}}}\right) \\
(\%)\end{array}$ & $\begin{array}{c}\text { Record } \\
\text { No. }\end{array}$ & $\begin{array}{l}\text { Previous } \\
\text { Record } \\
\text { No. }\end{array}$ \\
\hline 545161 & $\begin{array}{c}\text { Parallel } \\
\text { Perpendicular }\end{array}$ & 1 & 1340 & $\begin{array}{l}+0.33 \\
+0.49 \\
+0.30 \\
+0.41 \\
-0.14 \\
-0.09 \\
-0.24 \\
-0.19\end{array}$ & $\begin{array}{l}5641 \\
5643 \\
5645 \\
5647 \\
5642 \\
5644 \\
5646 \\
5648\end{array}$ & \\
\hline $\mathrm{B}_{4} \mathrm{C}-\mathrm{G}$ & Parallel & $i^{3}$ & $\left.\right|_{185} ^{1155}$ & $\begin{array}{l}-1.93 \\
-2.16 \\
-2.06 \\
-2.05 \\
-1.96 \\
-2.15 \\
-2.26 \\
-2.38 \\
-1.64 \\
-1.59 \\
-1.61 \\
-1.41 \\
-1.61 \\
-1.64 \\
-1.62 \\
-1.10\end{array}$ & $\begin{array}{l}6438 \\
6439 \\
6440 \\
6441 \\
6442 \\
6443 \\
6444 \\
6445 \\
6446 \\
6447 \\
6448 \\
6449 \\
6450 \\
6451 \\
6452 \\
6453\end{array}$ & \\
\hline $\mathrm{H}-419$ & $\begin{array}{l}\text { Perpendicular } \\
\text { Parallel }\end{array}$ & $\begin{array}{l}11.32 \\
11.32\end{array}$ & $\begin{array}{l}1510 \\
1510\end{array}$ & $\begin{array}{l}-0.54 \\
-1.09\end{array}$ & $\begin{array}{l}5621 \\
5622\end{array}$ & $\begin{array}{l}2836 \\
2843\end{array}$ \\
\hline $\mathrm{H}-315 \mathrm{~A}$ & $\begin{array}{l}\text { Perpendicular } \\
\text { Parallel }\end{array}$ & $\begin{array}{l}11.32 \\
11.32\end{array}$ & $\begin{array}{l}1510 \\
1510\end{array}$ & $\begin{array}{l}-2.42 \\
+2.57\end{array}$ & $\begin{array}{l}5623 \\
5624\end{array}$ & $\begin{array}{l}2833 \\
2835\end{array}$ \\
\hline $\mathrm{H}-328$ & $\begin{array}{c}\text { Parallel } \\
\text { Perpendicular }\end{array}$ & $\begin{array}{l}11.32 \\
11.32\end{array}$ & $\begin{array}{l}1510 \\
1510\end{array}$ & $\begin{array}{l}-2.38 \\
-2.98\end{array}$ & $\begin{array}{l}5627 \\
5628\end{array}$ & $\begin{array}{l}2841 \\
2832\end{array}$ \\
\hline $\mathrm{CHN}$ & $\begin{array}{c}\text { Parallel } \\
\text { Perpendicular }\end{array}$ & $\begin{array}{l}11.32 \\
11.32\end{array}$ & $\begin{array}{l}1510 \\
1510\end{array}$ & $\begin{array}{l}-7.32 \\
+4.21\end{array}$ & $\begin{array}{l}5631 \\
5632\end{array}$ & $\begin{array}{l}2845 \\
2834\end{array}$ \\
\hline $\mathrm{H}-319$ & $\begin{array}{c}\text { Parallel } \\
\text { Perpendicular }\end{array}$ & $\begin{array}{l}11.32 \\
11.32\end{array}$ & $\begin{array}{l}1510 \\
1510\end{array}$ & $\begin{array}{l}-2.26 \\
+0.57\end{array}$ & $\begin{array}{l}5633 \\
5634\end{array}$ & $\begin{array}{l}2847 \\
2848\end{array}$ \\
\hline$H-430$ & $\begin{array}{l}\text { Perpendicular } \\
\text { Parallel }\end{array}$ & $i^{6.78}$ & 1 & $\begin{array}{l}-0.71 \\
-0.53 \\
-0.93 \\
-2.31 \\
-2.47 \\
-0.74\end{array}$ & $\begin{array}{l}5635 \\
5636 \\
5637 \\
5638 \\
5651 \\
5652\end{array}$ & $\begin{array}{l}2846 \\
2837 \\
2838 \\
2844 \\
2831 \\
2840\end{array}$ \\
\hline $\mathrm{H}-451 \mathrm{C}$ & Parallel & $i^{3}$ & $\stackrel{960}{1}$ & $\begin{array}{l}-0.93 \\
-0.41 \\
-0.67\end{array}$ & $\begin{array}{l}5768 \\
5769 \\
5770\end{array}$ & \\
\hline
\end{tabular}


TABLE 7 (Continued)

\begin{tabular}{|c|c|c|c|c|c|c|}
\hline Grade & Orientation & $\begin{array}{l}\text { Total Fluence } \\
\times 10^{-21}\left(\mathrm{n} / \mathrm{cm}^{2}\right) \\
(\mathrm{E}>0.18 \mathrm{MeV})\end{array}$ & $\begin{array}{c}\text { Average } \\
\text { Irradiation } \\
\text { Temperature } \\
\left({ }^{\circ} \mathrm{C}\right)\end{array}$ & $\begin{array}{c}\text { Dimensional } \\
\text { Change } \\
1 \mathrm{n}\left(1+\frac{\Delta \mathrm{L}}{\mathrm{L}_{\mathrm{o}}}\right) \\
(\%)\end{array}$ & $\begin{array}{c}\text { Record } \\
\text { No. }\end{array}$ & $\begin{array}{c}\text { Previous } \\
\text { Record } \\
\text { No. }\end{array}$ \\
\hline $\begin{array}{c}\text { IH-451 } \\
\text { (char- } \\
\text { loaded) } \\
\\
\text { IH-451 } \\
\text { (contro1 } \\
\text { samples) }\end{array}$ & Paralle1 & $i^{3.4}$ & 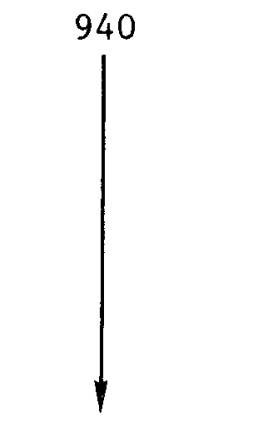 & $\begin{array}{l}-0.59 \\
-0.63 \\
-0.58 \\
-0.56 \\
-0.54 \\
-0.66 \\
-0.66 \\
-0.66 \\
-0.69 \\
-0.70\end{array}$ & $\begin{array}{l}6461 \\
6462 \\
6463 \\
6464 \\
6465 \\
6466 \\
6467 \\
6468 \\
6469 \\
6470\end{array}$ & \\
\hline
\end{tabular}




\subsection{COMPARISON}

Table 8 compares the dimensional changes observed in $\mathrm{H}-451, \mathrm{H}-327$, and $\mathrm{P}_{3}$ JHAN graphites irradiated under identical conditions. The $\mathrm{H}-451$ was the most stable of the three graphites, while the $\mathrm{P}_{3}$ JHAN clearly exhibited the largest dimensional changes. At moderate temperatures and fluence, $\mathrm{H}-327$ behaved in a manner comparable to $\mathrm{H}-451$, but it exhibited more dimensional change at higher temperatures and fluences.

\subsection{CARBON INSULATING MATERIALS}

A few samples of carbon insulating materials were irradiated in capsule OG-1. The materials were a rigid carbon foam (FPA-20), a flexible graphite foil (Grafoil), and a resilient layered fibrous carbon (Palarite C). Postirradiation dimensions were measured from photographs, and the results are shown in Table 9. All samples retained their integrity except the small (0.18-in. diameter) cores of Palarite $\mathrm{C}$, which were fragile and one of which became delaminated into pieces. The results may be summarized as follows:

FPA-20 Carbon Foam. Shrank $6 \%$ to $11 \%$ in both directions after irradiation to $3.1 \times 10^{21} \mathrm{n} / \mathrm{cm}^{2}$ at $900^{\circ} \mathrm{C}$.

Grafoil. Retained some flexibility. Less than $5 \%$ in-plane shrinkage after irradiation to $3.6 \times 10^{21} \mathrm{n} / \mathrm{cm}^{2}$ at $1350^{\circ} \mathrm{C}$.

Palarite C. Retained some resiliency. Shrank $11 \%$ parallel to the layers after irradiation to $3.1 \times 10^{21} \mathrm{n} / \mathrm{cm}^{2}$ at $900^{\circ} \mathrm{C}$ or $3.6 \times 10^{21} \mathrm{n} / \mathrm{cm}^{2}$ at $500^{\circ}$ to $1200^{\circ} \mathrm{C}$. Insignificant dimensional change at 1.1 to $1.3 \times 10^{21} \mathrm{n} / \mathrm{cm}^{2}$ at $400^{\circ}$ to $700^{\circ} \mathrm{C}$. Expanded $15 \%$ perpendicular to the layers after irradiation to $3.1 \times 10^{21} \mathrm{n} / \mathrm{cm}^{2}$ at $900^{\circ} \mathrm{C}$. 
TABLE 8

DIMENSIONAL CHANGES IN $\mathrm{H}-451, \mathrm{H}-327$, AND $\mathrm{P}_{3}$ JHAN

\begin{tabular}{|c|c|c|c|c|c|c|c|}
\hline \multirow{3}{*}{$\begin{array}{l}\text { Average } \\
\text { Irradiation } \\
\text { Temperature } \\
\quad\left({ }^{\circ} \mathrm{C}\right)\end{array}$} & \multirow{3}{*}{$\begin{array}{c}\text { Total } \\
\text { Fluence } \times 10^{-21} \\
\left(\mathrm{n} / \mathrm{cm}^{2}\right)(E>0.18 \mathrm{MeV})\end{array}$} & \multicolumn{6}{|c|}{ Dimensional Change, $\ln \left(1+\frac{\Delta L}{\bar{L}_{0}}\right)(\%)$} \\
\hline & & \multicolumn{3}{|c|}{ Perpendicular } & \multicolumn{3}{|c|}{ Para1lel } \\
\hline & & $\mathrm{H}-451$ & $\mathrm{H}-327$ & $\mathrm{P}_{3}$ JHAN & $\mathrm{H}-451$ & $\mathrm{H}-327$ & $\mathrm{P}_{3}$ JHAN \\
\hline 595 & 1.6 & -0.09 & -0.06 & -0.13 & -0.12 & -0.15 & -0.20 \\
\hline 750 & 2.1 & -0.09 & & -0.12 & -0.15 & -0.13 & -0.28 \\
\hline 980 & 3.4 & -0.48 & -0.4 & -0.74 & -0.78 & -1.0 & -1.83 \\
\hline 1020 & 3.4 & -0.49 & -0.46 & -0.80 & -0.90 & -1.22 & -2.03 \\
\hline 1325 & 3.6 & -1.57 & & -1.61 & -2.34 & & -4.06 \\
\hline
\end{tabular}


TABLE 9

CARBON INSULATING MATERIALS IRRADIATED IN CAPSULE OG-2

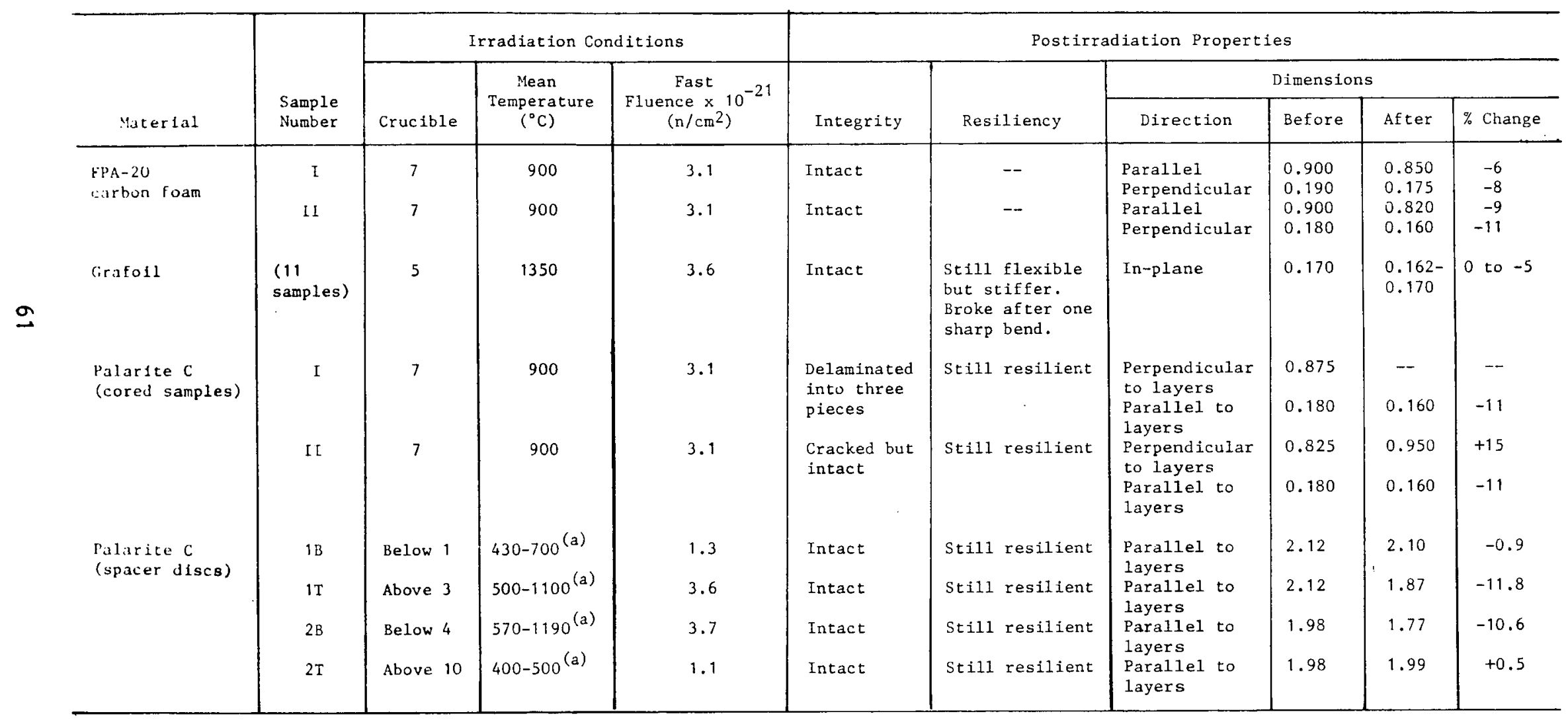

(a) Design temperature range, periphery to center line. 


\section{EXPERIMENTAL RESULTS - THERMAL PROPERTIES}

\subsection{THERMAL EXPANSIVITY}

Thermal expansivity measurements were made on 102 irradiated graphite samples $0.2 \mathrm{in.}$ in diameter by $0.45 \mathrm{in.}$ long. Measurements were made in a silica dilatometer between room temperature and $100^{\circ} \mathrm{C}$ less than the irradiation temperature. Graphites included $\mathrm{H}-327, \mathrm{H}-451$, and $\mathrm{H}-429$. The H-429 samples and some of the H-327 samples had been irradiated previously in capsule GEH-13-422; the H-429 samples provided high-fluence data on a near-isotropic petroleum-coke-based material.

The mean expansivities between room temperature and $500^{\circ} \mathrm{C}$ are 1 isted in Tables 10 and 11 . The expansivity of $H-327$ graphite is plotted as a function of fast neutron fluence in Fig. 6, and that of $\mathrm{H}-429$ and $\mathrm{H}-451$ is shown in Fig. 7. Neutron-induced changes in the thermal expansivity of H-327 graphite were very small (Fig. 6). There was some indication of a downward drift in the perpendicular expansivity, but the parallel expansivity was apparently unaffected. The near-isotropic graphites ( $\mathrm{H}-429$ and $\mathrm{H}-451$ ) (Fig. 7) showed a small increase in thermal expansivity at irradiation temperatures below about $900^{\circ} \mathrm{C}$ and a slight decrease in expansivity for the highest irradiation temperatures $\left(1370^{\circ}\right.$ to $\left.1500^{\circ} \mathrm{C}\right)$. At intermediate temperatures, there was no systematic trend. These changes are much smaller than those occurring in Gilsocoke-based graphites, whose thermal expansivities first increase and later decrease to about half their preirradiation value at around $5 \times 10^{21} \mathrm{n} / \mathrm{cm}^{2}$ ( $E>0.18 \mathrm{MeV}$ ) for temperatures in the vicinity of $1000^{\circ} \mathrm{C}$. 
TABLE 10

THERMAL EXPANSIVITY OF H-327 GRAPHITE SPECIMENS

IRRADIATED IN CAPSULE OG-1

\begin{tabular}{|c|c|c|c|c|c|c|}
\hline $\begin{array}{c}\text { Specimen } \\
\text { No. }\end{array}$ & $\begin{array}{l}\text { Orientation } \\
\text { and } \\
\text { Location }\end{array}$ & $\begin{array}{c}\text { Crucible } \\
\text { No. }\end{array}$ & $\begin{array}{r}\text { Hole } \\
\text { No. }\end{array}$ & $\begin{array}{c}\text { Mean } \\
\text { Irradiation } \\
\text { Temperature } \\
\left({ }^{\circ} \mathrm{C}\right)\end{array}$ & $\begin{array}{c}\text { F1uence } \times 10^{-21} \\
\left(\mathrm{n} / \mathrm{cm}^{2}\right) \\
(\mathrm{E}>0.18 \mathrm{MeV})\end{array}$ & $\begin{array}{l}\text { Coefficient } \\
\text { of Thermal } \\
\text { Expansivity } \\
\alpha \times 10^{6 \circ} \mathrm{C}^{-1} \\
\left(22^{\circ}-500^{\circ} \mathrm{C}\right)\end{array}$ \\
\hline $\begin{array}{r}1 \\
2 \\
201 \\
202 \\
101 \\
102 \\
251 \\
252\end{array}$ & $\begin{array}{l}\text { C para } \\
\text { C para } \\
\text { E para } \\
\text { E para } \\
\text { C perp } \\
\text { C perp } \\
\text { E perp } \\
\text { E perp }\end{array}$ & 1 & $\begin{array}{r}2 \\
3 \\
40 \\
40 \\
2 \\
3 \\
40 \\
40\end{array}$ & $\begin{array}{l}640 \\
640 \\
580 \\
580 \\
640 \\
640 \\
580 \\
580\end{array}$ & $i^{2}$ & $\begin{array}{l}1.84 \\
1.87 \\
1.72 \\
1.65 \\
3.47 \\
3.25 \\
3.55 \\
3.54\end{array}$ \\
\hline $\begin{array}{l}.226 . \\
.227 . \\
.228 . \\
.276 . \\
.277 . \\
.278 . \\
176 \\
177 \\
174 \\
175\end{array}$ & $\begin{array}{l}\text { E para } \\
\text { E para } \\
\text { E para } \\
\text { E perp } \\
\text { E perp } \\
\text { E perp } \\
\text { C para } \\
\text { E para } \\
C \text { perp } \\
\text { E perp }\end{array}$ & 1 & $\begin{array}{l}41 \\
41 \\
43 \\
41 \\
41 \\
43 \\
19 \\
19 \\
19 \\
19\end{array}$ & $\mid$ & $\dot{1}^{2.6}$ & $\begin{array}{l}1.72 \\
1.68 \\
2.05 \\
3.61 \\
3.72 \\
3.70 \\
2.03 \\
2.27 \\
3.18 \\
3.70\end{array}$ \\
\hline $\begin{array}{l}222 \\
223 \\
224 \\
272 \\
273 \\
274\end{array}$ & $\begin{array}{l}\text { E para } \\
E \text { para } \\
E \text { para } \\
E \text { perp } \\
E \text { perp } \\
E \text { perp }\end{array}$ & 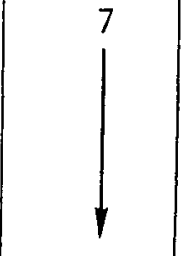 & $\begin{array}{l}2 \\
3 \\
4 \\
2 \\
3 \\
4\end{array}$ & $\stackrel{980}{1}$ & $i^{3.1}$ & $\begin{array}{l}1.97 \\
1.96 \\
1.93 \\
3.10 \\
3.38 \\
3.27\end{array}$ \\
\hline $\begin{array}{l}146 \\
150 \\
147 \\
151 \\
148 \\
152 \\
149 \\
153\end{array}$ & $\begin{array}{l}\text { C perp } \\
C \text { perp } \\
\text { E perp } \\
\text { E perp } \\
\text { C para } \\
\text { C para } \\
\text { E para } \\
\text { E para }\end{array}$ & $\begin{array}{l}4 \\
1\end{array}$ & $\begin{array}{l}35 \\
36 \\
35 \\
36 \\
35 \\
36 \\
35 \\
36\end{array}$ & $\begin{array}{l}1050-1325 \\
1050-1275 \\
1050-1325 \\
1050-1275 \\
1050-1325 \\
1050-1275 \\
1050-1325 \\
1050-1275\end{array}$ & $\left.\right|^{9.9}$ & $\begin{array}{l}2.99 \\
3.24 \\
3.59 \\
3.48 \\
2.05 \\
1.85 \\
1.87 \\
1.58\end{array}$ \\
\hline $\begin{array}{l}240 \\
241\end{array}$ & $\begin{array}{l}\text { E para } \\
\text { E perp }\end{array}$ & $\begin{array}{l}5 \\
5\end{array}$ & $\begin{array}{l}20 \\
20\end{array}$ & $\begin{array}{l}1370-1575 \\
1370-1575\end{array}$ & $\begin{array}{l}11.3 \\
11.3\end{array}$ & $\begin{array}{l}2.12 \\
3.04\end{array}$ \\
\hline
\end{tabular}

${ }^{(a)} \mathrm{C}=$ center, $\mathrm{E}=$ edge; para - parallel, perp = perpendicular. 
TABLE 11.

THERMAL EXPANSIVITY OF $11-429$ AIJ II-451 GRAPIITE SPECIMENS IRRADIATED IN CAPSULE OG- 1

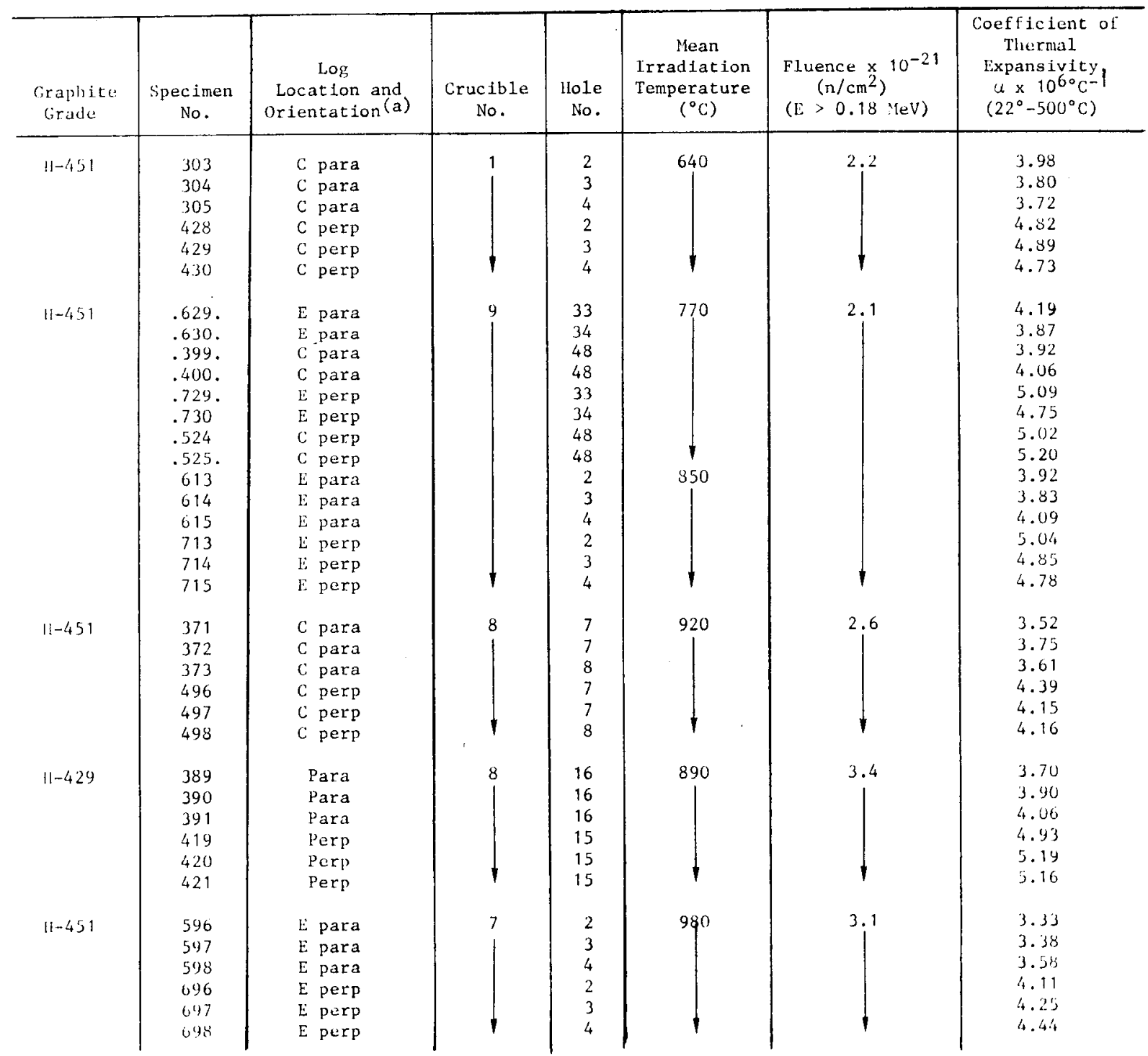


TABLE 11 (Continued)

\begin{tabular}{|c|c|c|c|c|c|c|c|}
\hline $\begin{array}{l}\text { Graphite } \\
\text { Grale }\end{array}$ & $\begin{array}{c}\text { Specimen } \\
\text { No. }\end{array}$ & $\begin{array}{l}\text { Log } \\
\text { Location and } \\
\text { Urientation }(a)\end{array}$ & $\begin{array}{c}\text { Crucible } \\
\text { No. }\end{array}$ & $\begin{array}{l}\text { Hole } \\
\text { No. }\end{array}$ & $\begin{array}{c}\text { Mean } \\
\text { Irradiation } \\
\text { Temperature } \\
\left({ }^{\circ} \mathrm{C}\right)\end{array}$ & $\begin{array}{c}\text { Fluence } x_{2} 10^{-21} \\
\left(\mathrm{n} / \mathrm{cm}^{2}\right) \\
(E>0.18 \mathrm{iev})\end{array}$ & $\begin{array}{c}\text { Coefficient of } \\
\text { lliermal } \\
\text { Expansivity } \\
4 \times 10^{6} \mathrm{C}^{-1} \\
\left(22^{\circ}-500^{\circ} \mathrm{C}\right)\end{array}$ \\
\hline $11-451$ & $\begin{array}{l}.355 . \\
.356 . \\
.357 . \\
.480 \\
.481 . \\
.482 .\end{array}$ & $\begin{array}{l}\text { C para } \\
\text { C para } \\
\text { C para } \\
\text { C perp } \\
\text { C perp } \\
\text { C perp }\end{array}$ & $i_{1}^{6}$ & $\begin{array}{l}6 \\
6 \\
7 \\
6 \\
6 \\
7\end{array}$ & 1060 & $3 i^{4}$ & $\begin{array}{l}3.01 \\
3.76 \\
3.79 \\
4.52 \\
4.37 \\
4.08\end{array}$ \\
\hline $11-451$ & $\begin{array}{l}583 \\
584 \\
585 \\
683 \\
684 \\
685\end{array}$ & $\begin{array}{l}\text { E para } \\
E \text { para } \\
\text { E para } \\
\text { E perp } \\
\text { E perp } \\
\text { E perp }\end{array}$ & 4 & $\begin{array}{l}2 \\
2 \\
3 \\
2 \\
2 \\
2 \\
3\end{array}$ & 1 & $i^{3.7}$ & $\begin{array}{l}3.49 \\
3.55 \\
3.64 \\
3.70 \\
4.01 \\
3.78\end{array}$ \\
\hline $11-429$ & $\begin{array}{l}404 \\
405 \\
406 \\
434 \\
435 \\
436\end{array}$ & $\begin{array}{l}\text { Para } \\
\text { Para } \\
\text { Para } \\
\text { Perp } \\
\text { Perp } \\
\text { Perp }\end{array}$ & 4 & $\begin{array}{l}30 \\
30 \\
30 \\
30 \\
30 \\
40\end{array}$ & $\frac{1050-1275}{\mid}$ & $i^{6.3}$ & $\begin{array}{l}3.77 \\
3.67 \\
3.66 \\
4.70 \\
4.52 \\
4.37\end{array}$ \\
\hline $\mid 1-451$ & $\begin{array}{l}341 \\
342 \\
343 \\
466 \\
467 \\
468\end{array}$ & $\begin{array}{l}\text { C para } \\
\text { C para } \\
\text { C para } \\
\text { C perp } \\
\text { C perp } \\
\text { C perp }\end{array}$ & 1 & $\begin{array}{l}6 \\
6 \\
7 \\
6 \\
6 \\
7\end{array}$ & 1390 & $3 i^{6}$ & $\begin{array}{l}3.49 \\
3.73 \\
3.69 \\
4.30 \\
4.37 \\
4.16\end{array}$ \\
\hline $11-429$ & $\begin{array}{l}141 \\
142 \\
143 \\
161 \\
162 \\
163\end{array}$ & $\begin{array}{l}\text { Para } \\
\text { Para } \\
\text { Para } \\
\text { Perp } \\
\text { Perp } \\
\text { Perp }\end{array}$ & $\int^{5}$ & $\begin{array}{l}16 \\
16 \\
17 \\
16 \\
16 \\
17\end{array}$ & $1370-1500$ & $i_{1}^{6.6}$ & $\begin{array}{l}3.58 \\
3.38 \\
3.05 \\
4.16 \\
4.07 \\
4.29\end{array}$ \\
\hline
\end{tabular}

${ }^{(a)}{ }_{C}=$ center, $E=$ edge; para = parallel, perp = perpendicular. 


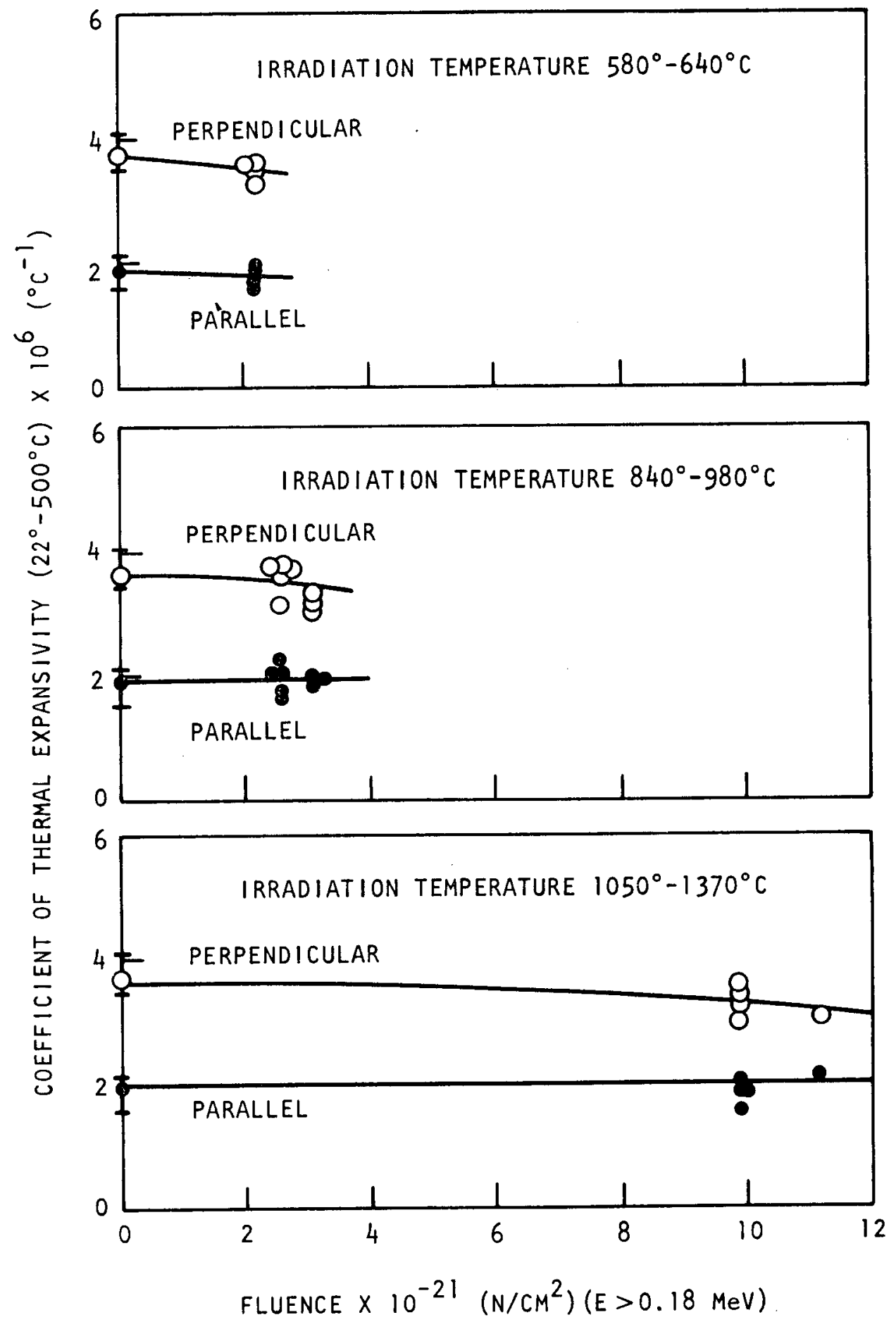

Fig. 6. Thermal expansivity of $\mathrm{H}-327$ graphite as a function of fast neutron fluence 


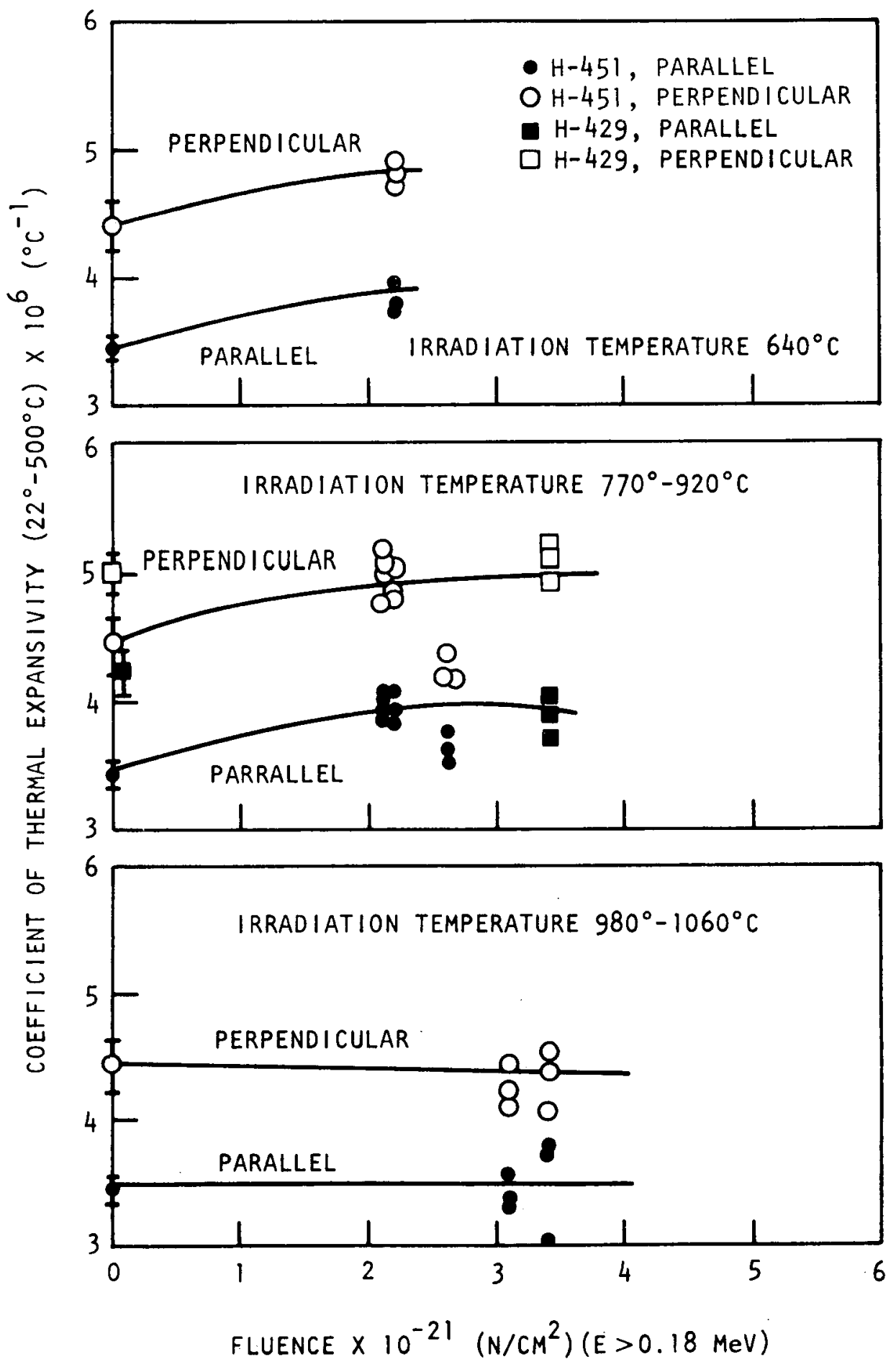

Fig. 7a. Thermal expansivity of $\mathrm{H}-451$ and $\mathrm{H}-429$ graphites irradiated at $640^{\circ}$ to $1060^{\circ} \mathrm{C}$ as a function of fast neutron fluence 


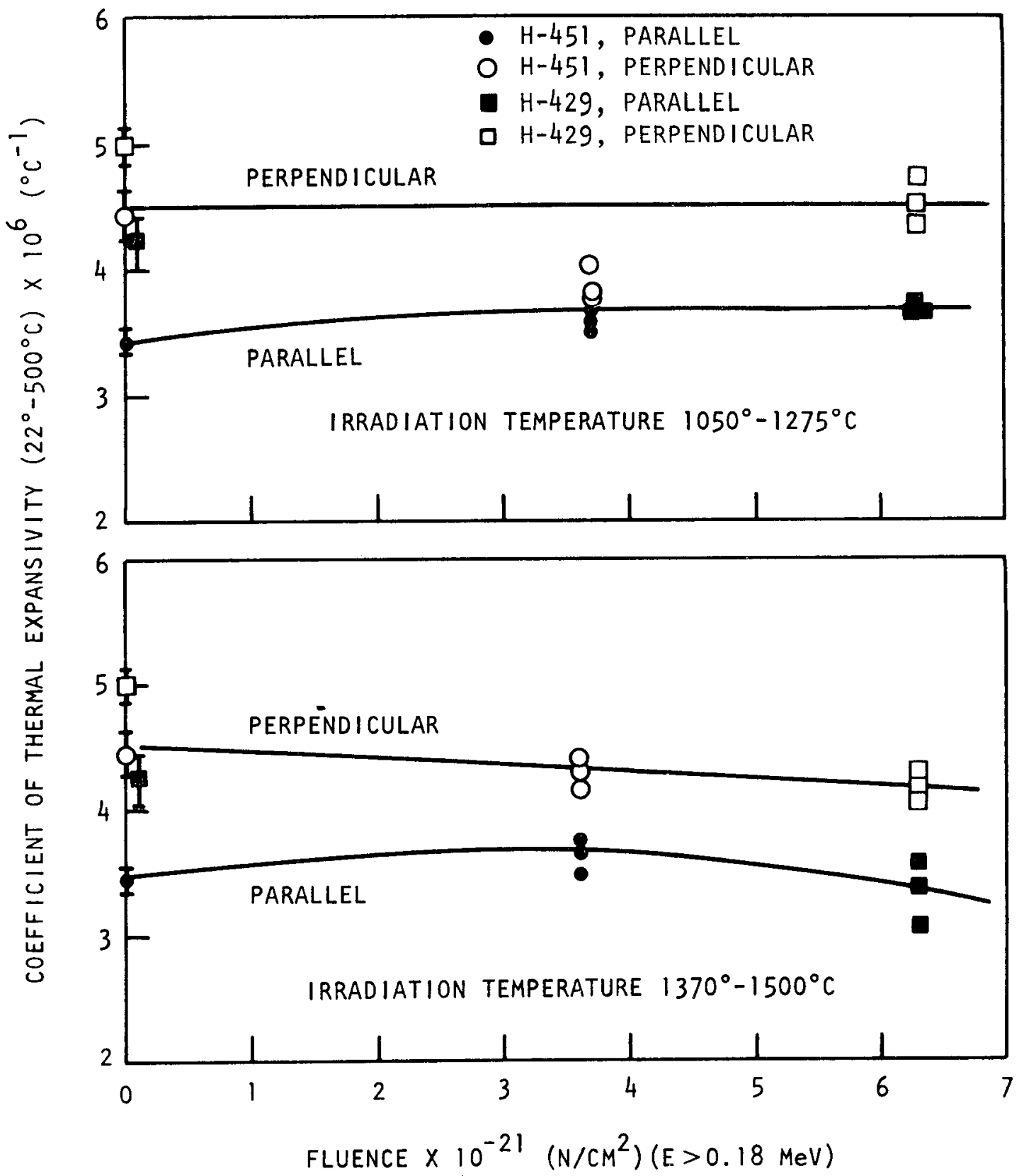

Fig. 7b. Thermal expansivity of $\mathrm{H}-451$ and $\mathrm{H}-429$ graphites irradiated at $1050^{\circ}$ to $1500^{\circ} \mathrm{C}$ as a function of fast neutron fluence 
6.2. THERMAL CONDUCTIVITY

Thermal diffusivity measurements were made on 87 discs $(0.45$ in. in diameter by 0.05 in. thick) of $\mathrm{H}-327$ and $\mathrm{H}-451$ graphites after irradiation in the OG-1 capsule. The H-327 discs had previously been irradiated in capsule GEH-13-422. Thermal diffusivity measurements were made by the heat-pulse method (ASTM Method of Test C-741), and the thermal diffusivity values were converted to thermal conductivities by multiplying by the density and literature values for the heat capacity of graphite. About half the samples were tested at room temperature only, and half were measured with the temperature increasing in $100^{\circ} \mathrm{C}$ steps to $100^{\circ} \mathrm{C}$ less than the irradiation temperature, or to $800^{\circ} \mathrm{C}$, whichever was lower.

The results are listed in Tables 12 and 13 . The conductivity at the irradiation temperature was estimated by extrapolating the data points, assuming a temperature dependence curve appropriate for irradiated graphite (Ref. 4). The conductivities at room temperature and at the irradiation temperature are plotted as a function of fluence in Figs. 8 through 11; these figures also include data from samples irradiated earlier in capsule GEH-13-422. Because of its high degree of orientation, the H-327 graphite had a higher conductivity parallel to extrusion than perpendicular to extrusion. For H-451 graphite, the difference was much less marked. At $600^{\circ}$ and $920^{\circ} \mathrm{C}$, the conductivity of irradiated $\mathrm{H}-451$ in the perpendicular direction was considerably greater than that of irradiated $\mathrm{H}-327$; the $\mathrm{H}-451$ conductivity in the perpendicular direction was similar to that of $\mathrm{H}-327$ in the parallel direction.

Typical examples of the thermal conductivity of irradiated $\mathrm{H}-327$ and H-451 graphites as a function of measurement temperature are shown in Fig. 12. Irradiation reduces both the level and the temperature sensitivity of the thermal conductivity, the effect being most pronounced for low irradiation temperatures. 
TABLE 12

THERMAL CONDUCTIVITY OF 11-327 GRAPHITE SPECIMENS IRRADIATED IN CAPSULE OG-1

\begin{tabular}{|c|c|c|c|c|c|c|c|c|c|c|}
\hline \multirow[b]{2}{*}{$\begin{array}{c}\text { specimen } \\
\text { iso. }\end{array}$} & \multirow[b]{2}{*}{$\begin{array}{l}\text { Log } \\
\text { location and } \\
\text { Irientation(a) }\end{array}$} & \multirow[b]{2}{*}{$\begin{array}{c}\text { Crucible } \\
\text { No. }\end{array}$} & \multirow[b]{2}{*}{$\begin{array}{c}\text { Mean } \\
\text { Irradiation } \\
\text { Temperature } \\
\left({ }^{\circ}{ }^{\circ}\right)\end{array}$} & \multirow[b]{2}{*}{$\begin{array}{c}\text { Eluence } \times 10^{-21} \\
\left(\mathrm{n} / \mathrm{cm}^{2}\right) \\
(E>0.18 \mathrm{MeV})\end{array}$} & \multicolumn{6}{|c|}{ Thermal Conductivity $\left(\mathrm{ca} 1 / \mathrm{cm}-\mathrm{sec}-{ }^{\circ} \mathrm{C}\right)$} \\
\hline & & & & & $22^{\circ} \mathrm{C}$ & $200^{\circ} \mathrm{C}$ & $400^{\circ} \mathrm{C}$ & $600^{\circ} \mathrm{C}$ & $800^{\circ} \mathrm{C}$ & $\begin{array}{l}\text { Irridiated } \\
\text { Temperature } \\
\text { (extripoliated) }\end{array}$ \\
\hline 1 & E perp & 1 & $600-625$ & 2.9 & 0.038 & 0.044 & 0.044 & & & 0.044 \\
\hline 2 & E perp & $i$ & $600-625$ & 2.9 & 0.038 & 0.044 & 0.043 & & & 0.041 \\
\hline 13 & I: perp & 1 & $600-625$ & 2.9 & 0.040 &.- & - & & & - \\
\hline 14 & L $p \in \cdot r p$ & 1 & $600-625$ & 2.9 & 0.052 & 0.061 & 0.060 & & & 0.055 \\
\hline is & E perp & 1 & $600-625$ & 2.9 & 0.042 & -- & -- & & & -- \\
\hline 65 & E perp & 1 & $600-625$ & 2.5 & 0.047 & 0.054 & 0.054 & & & 0.050 \\
\hline 66 & I: perp & 1 & $600-625$ & 2.5 & 0.055 & 0.062 & 0.057 & & & 0.053 \\
\hline 81 & J: pal:a & 1 & $600-625$ & 2.9 & 0.059 & 0.066 & 0.070 & & & 0.003 \\
\hline 82 & ¿ para & 1 & $600-625$ & 2.9 & 0.064 & 0.081 & 0.080 & & & 0.071 \\
\hline ys & E para & 1 & $600-625$ & 2.9 & 0.082 & 0.096 & 0.099 & & & 0.095 \\
\hline 94 & E para & 1 & $600-625$ & 2.9 & 0.074 & -- & -- & & & -- \\
\hline 95 & E para & 1 & $600-625$ & 2.9 & 0.076 & -- & -- & & & $\sim$ \\
\hline 06 & E para & 1 & $600-625$ & 2.9 & 0.086 & -- & -- & & & -- \\
\hline 97 & E para & 1 & $600-625$ & 2.9 & 0.073 & -- & -- & & & -- \\
\hline 116 & E par:1 & 1 & $600-625$ & 2.5 & 0.080 & 0.094 & 0.091 & & & 0.087 \\
\hline 43 & E perp & 5 & $1225-1350$ & 9.9 & 0.133 & 0.159 & 0.143 & 0.127 & 0.112 & 0.085 \\
\hline 44 & l: perp & 5 & $1225-1350$ & 9.9 & 0.131 & 0.136 & 0.118 & 0.105 & 0.093 & 0.078 \\
\hline 43 & L perp & 5 & $1225-1350$ & 9.9 & 0.149 & -- & -- & - & -- & -- \\
\hline 46 & E perp & 5 & $1225-1350$ & 9.9 & 0.137 & 0.130 & 0.117 & 0.104 & 0.094 & 0.078 \\
\hline 47 & E perp & 5 & $1225-1350$ & 9.9 & 0.129 & -- & -- & -- & -- & -- \\
\hline 69 & E perp & 5 & $1300-1350$ & 6.2 & 0.174 & 0.166 & 0.153 & 0.136 & 0.121 & 0.090 \\
\hline 70 & E purp & 5 & $1300-1350$ & 6.2 & 0.175 & 0.189 & 0.173 & 0.152 & 0.136 & 0.105 \\
\hline 86 & E pira & 5 & $1225-1350$ & 9.9 & 0.212 & 0.222 & 0.195 & 0.176 & 0.139 & 0.10 \\
\hline 87 & L para & 5 & $1225-1350$ & 9.9 & 0.198 & 0.219 & 0.203 & 0.170 & 0.144 & 0.10 \\
\hline 38 & E para & 5 & $1225-1350$ & 9.9 & 0.189 & 0.192 & 0.179 & 0.150 & 0.141 & 0.11 \\
\hline 103 & E para & 5 & $1225-1350$ & 9.9 & 0.179 & -- & -- & -- & - & - \\
\hline 104 & E para & 5 & $1225-1350$ & 9.9 & 0.176 & -- & -- & -- & -- & $\rightarrow$ \\
\hline 119 & E para & 5 & $1300-1350$ & 6.2 & 0.198 & 0.201 & 0.181 & 0.167 & 0.144 & 0.11 \\
\hline 120 & E para & 5 & $1300-1350$ & 6.2 & 0.199 & 0.220 & 0.188 & 0.176 & 0.153 & 0.12 \\
\hline 3 & l: perp & 7 & $875-920$ & 5.1 & 0.079 & 0.097 & 0.083 & 0.078 & 0.072 & 0.070 \\
\hline$\therefore$ & E perp & 7 & $875-920$ & 5.1 & 0.072 & 0.076 & 0.071 & 0.066 & 0.058 & 0.055 \\
\hline 32 & L perp & 7 & $875-920$ & 5.1 & 0.085 & -- & -- & - & -- & - \\
\hline 13. & l: perp & 7 & $375-920$ & 5.1 & 0.077 & 0.086 & 0.077 & 0.061 & 0.056 & 0.453 \\
\hline 34 & $1:$ perp & 7 & $875-920$ & 5.1 & 0.082 & -- & -- & -- & -- & -- \\
\hline 35 & I: perp & 7 & $875-920$ & 5.1 & 0.077 & -- & -- & - & -- & $\rightarrow-$ \\
\hline 67 & E perp & 7 & $900-920$ & 3.9 & 0.078 & 0.087 & 0.083 & 0.072 & 0.061 & 0.060 \\
\hline S) & l: para & 7 & $900-920$ & 5.1 & 0.095 & 0.094 & 0.083 & 0.081 & 0.0777 & $11.1 \% \%$ \\
\hline 84 & lipara & 7 & $900-920$ & 5.1 & 0.094 & 0.110 & 0.100 & 0.094 & 0.045 & $0.01 \div 0$ \\
\hline 94 & E. pitfa & 7 & $900-920$ & 5.1 & 0.101 & 0.116 & 0.105 & 0.099 & 0.041 & נ.0.0 \\
\hline 100 & E para & 7 & $900-920$ & 5.1 & 0.100 & -- & $\rightarrow$ & -- & -- & $-\cdots$ \\
\hline 101 & E para & 7 & $900-420$ & 5.1 & 0.117 & -- & -- & - & -- & $\cdots$ \\
\hline 117 & E: para & 7 & $900-920$ & 3.9 & 0.108 & 0.135 & 0.133 & 0.123 & 0.108 & 1. 11, \\
\hline
\end{tabular}

(a) $E=2 d: c$; para = parallel; perp = perpendicular. 
TABLL 13

THERYAL CONDUCTIVITY OF 11-451 GRAPHITE SPLCLILIS IRRADIATLD IN CAPSULE OG-1

\begin{tabular}{|c|c|c|c|c|c|c|c|c|c|c|}
\hline \multirow[b]{2}{*}{$\begin{array}{c}\text { Speefmen } \\
\text { No. }\end{array}$} & \multirow[b]{2}{*}{ Orientation (a) } & \multirow[b]{2}{*}{$\begin{array}{c}\text { Crucible } \\
\text { No. }\end{array}$} & \multirow[b]{2}{*}{$\begin{array}{c}\text { Mean } \\
\text { Irradiation } \\
\text { Temperature } \\
\left({ }^{\circ} \mathrm{C}\right)\end{array}$} & \multirow[b]{2}{*}{$\begin{array}{c}\text { Fluence } \times 10^{-21} \\
\left(\mathrm{n} / \mathrm{cm}^{2}\right) \\
(E>0.18 \mathrm{MeV})\end{array}$} & \multicolumn{6}{|c|}{ Thermal Conductivity (cal $\left./ \mathrm{cm}-\sec -{ }^{n} \mathrm{C}\right)$} \\
\hline & & & & & $22^{\circ} \mathrm{C}$ & $200^{\circ} \mathrm{C}$ & $400^{\circ} \mathrm{C}$ & $600^{\circ} \mathrm{C}$ & $800^{\circ} \mathrm{C}$ & $\begin{array}{l}\text { Irradiated } \\
\text { lemperature } \\
\text { (extrapolated) }\end{array}$ \\
\hline 221 & lerp & 1 & 600 & 2.2 & 0.085 & 0.081 & 0.082 & & & 0.080 \\
\hline 222 & Perp & 1 & 600 & 2.2 & 0.078 & 0.083 & 0.080 & & & 0.077 \\
\hline 223 & Perp & 1 & 600 & 2.2 & 0.078 & 0.082 & 0.082 & & & 0.081 \\
\hline 224 & Perp & 1 & 600 & 2.2 & 0.077 & -- & -- & & & -- \\
\hline 225 & perp & 1 & 600 & 2.2 & 0.068 & -- & -- & & & -- \\
\hline 226 & Perp & 1 & 600 & 2.2 & 0.074 & -- & -- & & & -- \\
\hline 227 & Perp & 1 & 600 & 2.2 & 0.076 & -- & -- & & & -- \\
\hline 228 & Perp & 1 & 600 & 2.2 & 0.073 & -- & -- & & & -- \\
\hline 229 & Perp & 1 & 600 & 2.2 & 0.080 & 0.095 & 0.086 & & & 0.080 \\
\hline 230 & $p^{\prime} \in \mathrm{rp}$ & 1 & 600 & 2.2 & 0.077 & 0.086 & 0.080 & & & 0.073 \\
\hline 201 & Para & 1 & 600 & 2.2 & 0.079 & 0.083 & 0.080 & & & 0.075 \\
\hline 202 & Para & 1 & 600 & 2.2 & 0.087 & 0.095 & 0.097 & & & 0.084 \\
\hline 203 & Para & 1 & 600 & 2.2 & 0.083 & -- & -- & & & -- \\
\hline 204 & Para & 1 & 600 & 2.2 & 0.083 & - & -- & & & -- \\
\hline 242 & Perp & 5 & 1350 & 3.6 & 0.195 & 0.175 & 0.164 & 0.143 & 0.123 & 0.09 \\
\hline 243 & Perp & 5 & 1350 & 3.6 & 0.202 & 0.213 & 0.190 & 0.161 & 0.127 & 0.085 \\
\hline 244 & Perp & 5 & 1350 & 3.6 & 0.216 & 0.203 & 0.185 & 0.162 & 0.129 & 0.09 \\
\hline 245 & lerp & 5 & 1350 & 3.6 & 0.205 & -- & -- & -- & -- & - \\
\hline 246 & Perp & 5 & 1350 & 3.6 & 0.214 & -- & -- & -- & -- & -- \\
\hline 247 & Perp & 5 & 1350 & 3.6 & 0.202 & -- & $-c$ & -- & -- & -- \\
\hline 248 & Perp & 5 & 1350 & 3.6 & 0.205 & -- & -- & -- & -- & -- \\
\hline 243 & Perp & 5 & 1350 & 3.6 & 0.215 & -- & -- & -- & -- & -- \\
\hline 250 & Perp & 5 & 1350 & 3.6 & 0.162 & 0.173 & 0.158 & 0.144 & 0.131 & 0.105 \\
\hline 251 & Perp & 5 & 1350 & 3.6 & 0.179 & 0,182 & 0.173 & 0.151 & 0.134 & 0.10 \\
\hline 210 & l'ara & 5 & 1350 & 3.6 & 0.193 & 0.216 & 0.188 & 0.158 & 0.142 & 0.10 \\
\hline 211 & Para & 5 & 1350 & 3.6 & 0.195 & 0.209 & 0.182 & 0.159 & 0.143 & 0.10 \\
\hline 212 & Para & 5 & 1350 & 3.6 & 0.195 & -- & -- & -- & -- & -- \\
\hline 213 & Para & 5 & 1350 & 3.6 & 0.198 & -- & -- & -- & -- & -- \\
\hline 214 & Para & 5 & 1350 & 3.6 & 0.173 & 0.179 & 0.162 & 0.150 & 0.133 & 0.095 \\
\hline 231 & Perp & 7 & 920 & 3.1 & 0.104 & 0.131 & 0.112 & 0.101 & 0.092 & 0.090 \\
\hline 232 & Perp & 7 & 920 & 3.1 & 0.097 & 0.111 & 0.105 & 0.092 & 0.085 & 0.082 \\
\hline 233 & Perp & 7 & 920 & 3.1 & 0.109 & 0.113 & 0.100 & 0.092 & 0.082 & 0.080 \\
\hline 234 & Perp & 7 & 920 & 3.1 & 0.110 & -- & -- & -- & -- & -- \\
\hline 235 & Perp & 7 & 920 & 3.1 & 0.114 & $\cdots$ & -+ & -- & -- & -- \\
\hline 236 & Perp & 7 & 920 & 3.1 & 0.099 & -- & -- & -- & - & - \\
\hline 237 & Perp & 7 & 920 & 3.1 & 0.104 & -- & -- & -- & -- & -- \\
\hline 238 & Perp & 7 & 920 & 3.1 & 0.085 & -- & -- & -- & -- & -- \\
\hline 239 & Perp & 7 & 920 & 3.1 & 0.099 & 0.111 & 0.097 & 0.091 & 0.085 & 0.082 \\
\hline 240 & Perp & 7 & 920 & 3.1 & 0.106 & 0.120 & 0.113 & 0.104 & 0.096 & 0.092 \\
\hline 241 & Perp & 7 & 920 & 3.1 & 0.102 & -- & -- & -- & -- & -- \\
\hline 205 & Para & 7 & 920 & 3.1 & 0.117 & 0.132 & 0.123 & 0.110 & 0.100 & 0.095 \\
\hline 206 & Para & 7 & 920 & 3.1 & 0.118 & 0.130 & 0.117 & 0.107 & 0.096 & 0.092 \\
\hline 207 & Para & 7 & 920 & 3.1 & 0.112 & -- & -- & -- & -- & -- \\
\hline 208 & Para & 7 & 920 & 3.1 & 0.113 & -- & -- & -- & -- & - \\
\hline 209 & Para & 7 & 920 & 3.1 & 0.123 & 0.130 & 0.121 & 0.116 & 0.103 & 0.100 \\
\hline
\end{tabular}

(a) ${ }_{\text {Perp }}=$ perpendicular; Para = parallel. 


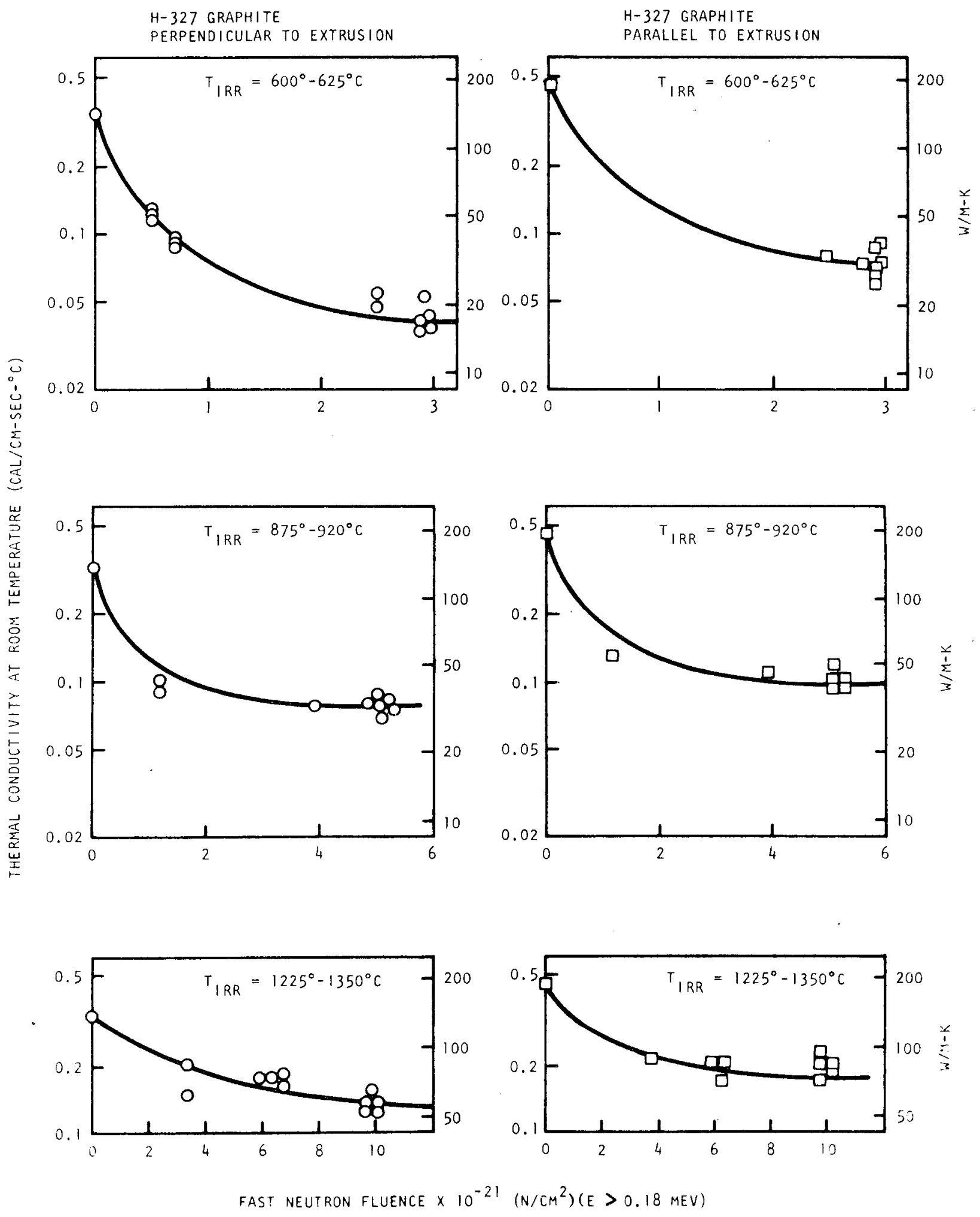

Fig. 8. Thermal conductivity at room temperature of H-327 graphite as a function of fast neutron fluence 


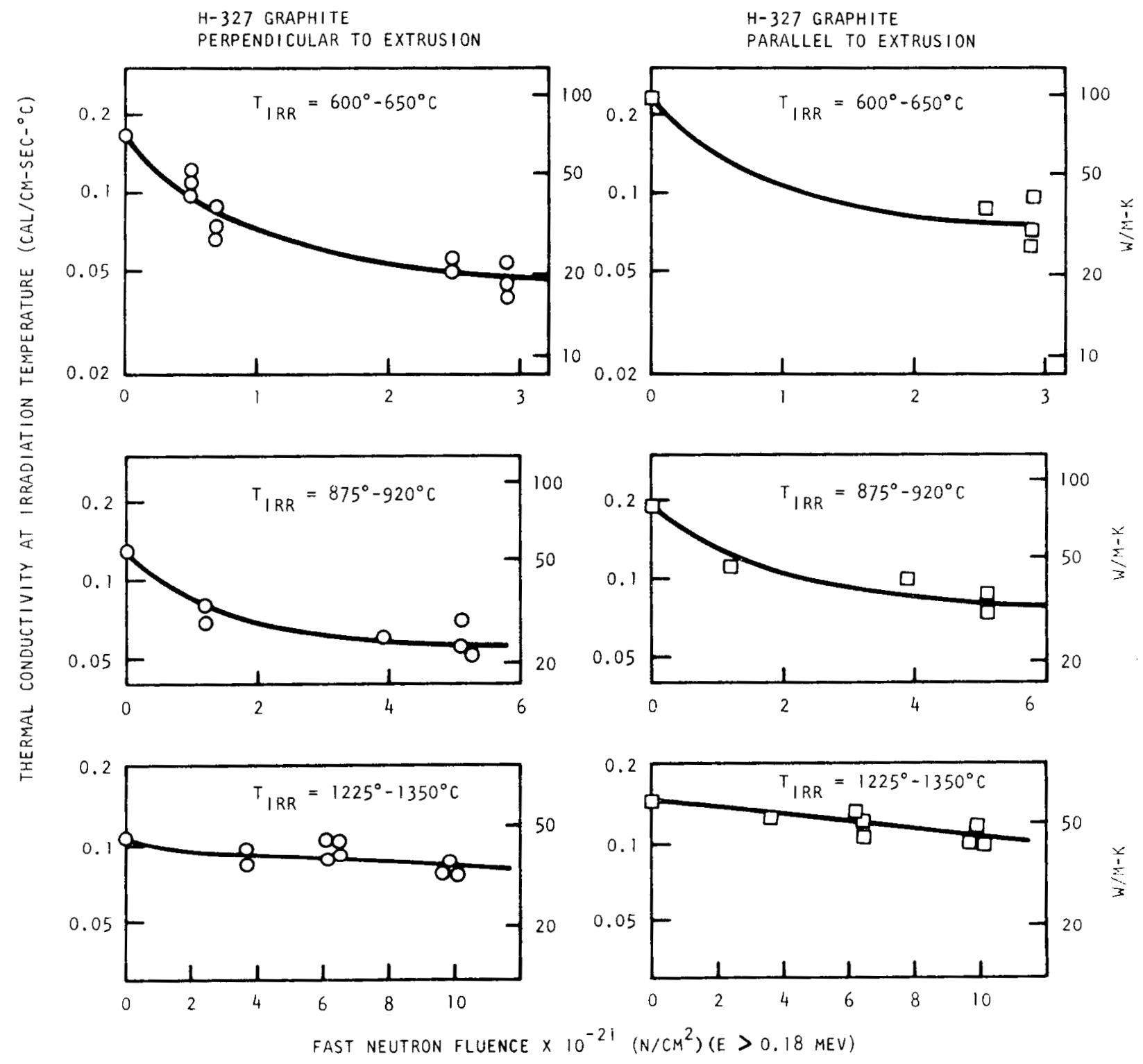

Fig. 9. Thermal conductivity at irradiation temperature of $\mathrm{H}-327$ graphite as a function of fast neutron fluence 


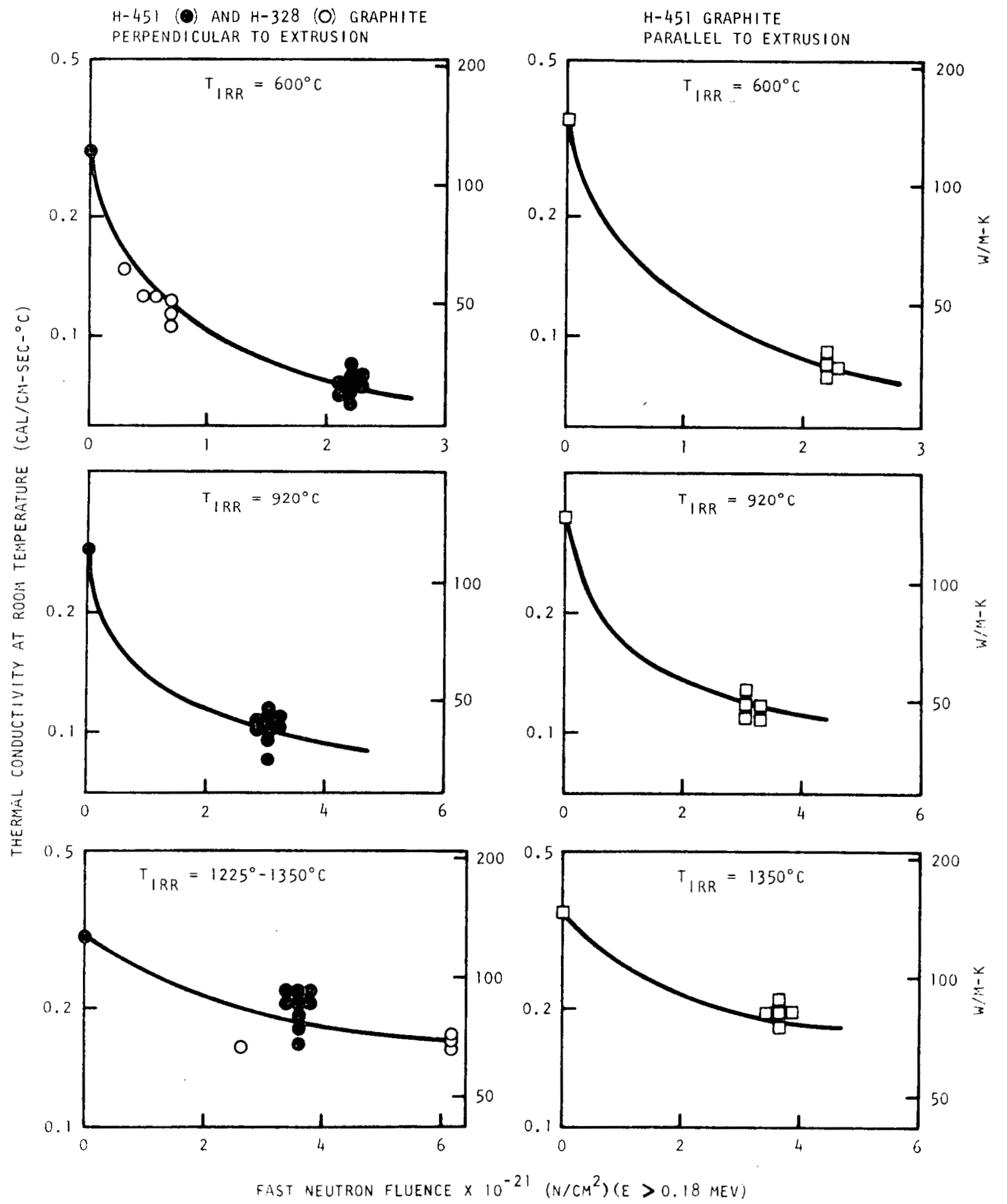

Fig. 10. Thermal conductivity at room temperature of H-451 graphite as a function of fast neutron fluence. (Some data points on Gilsocarbon H-328 graphite from capsule GEH-13-422 are included.) 


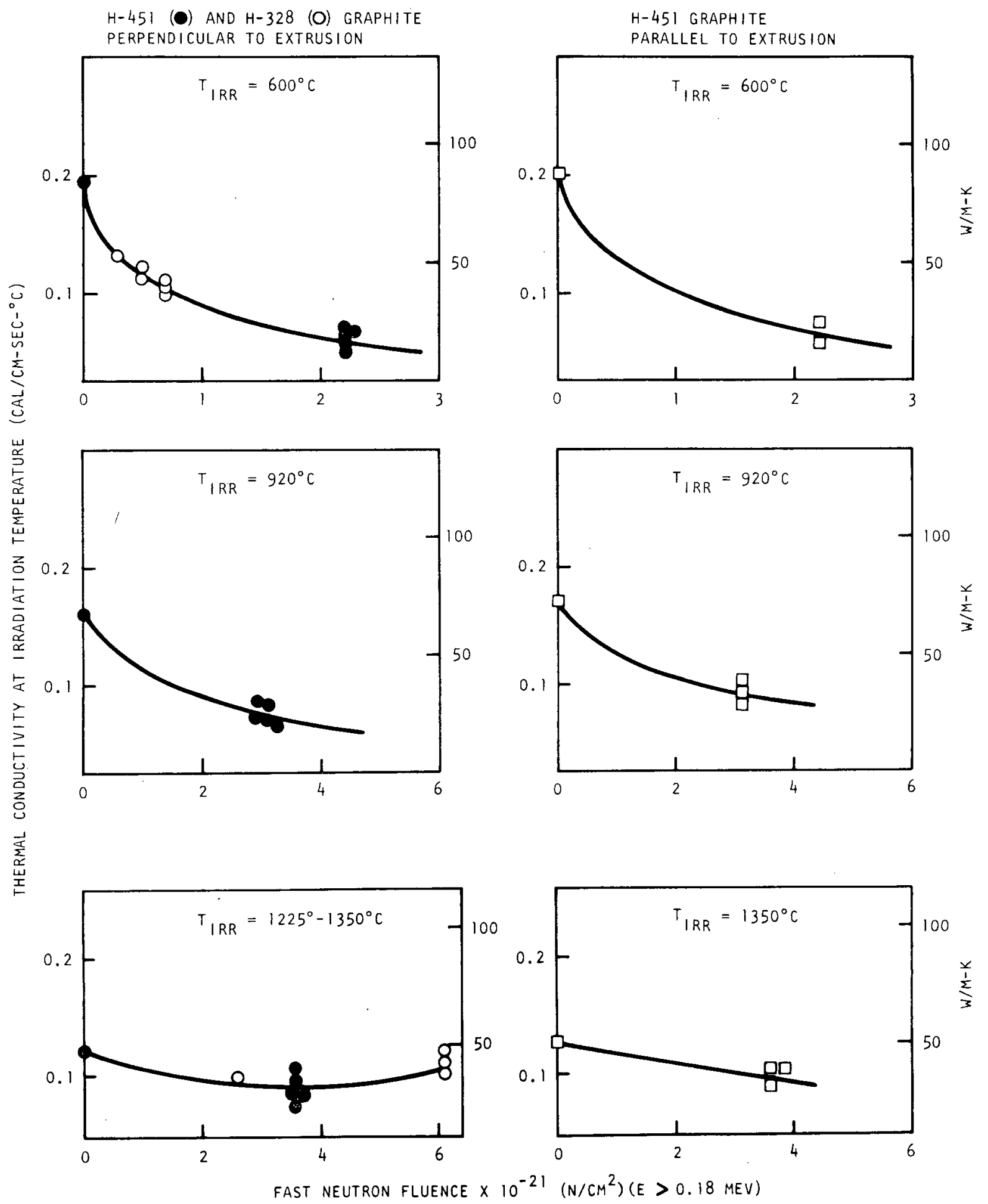

Fig. 11. Thermal conductivity at irradiation temperature of $\mathrm{H}-451$ graphite as a function of fast neutron fluence. (Some data points on Gilsocarbon $\mathrm{H}-328$ graphite from capsule GEH-13-422 are included.) 


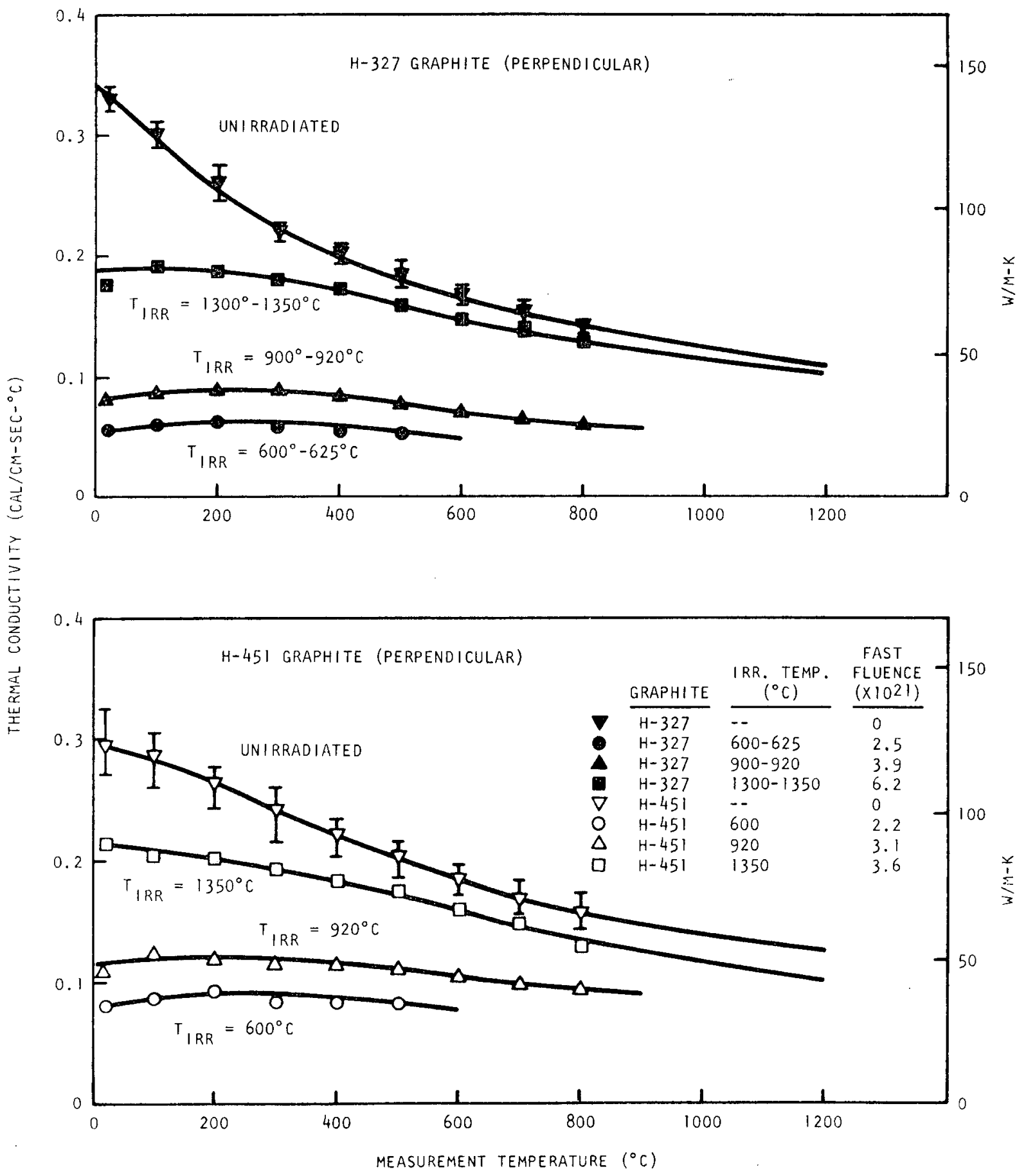

Fig. 12. Thermal conductivity of unirradiated and irradiated H-327 and H-451 graphites (perpendicular orientation) as a function of measurement temperature. (Unirradiated data show mean and range of 10 samples.) 
The thermal conductivity of the irradiated H-451 samples was in good agreement with values predicted earlier from published data on Gilsocarbon-based graphites (Ref. 4). However, the thermal conductivities of the H-327 samples Irradiated at $600^{\circ}$ and $900^{\circ} \mathrm{C}$ were $20 \%$ to $30 \%$ lower than the earlier predictions. There is no obvious explanation of why the conductivity of the needle-coke graphite should have been reduced more than that of the isotropic graphite. 


\section{EXPERIMENTAL RESULTS - TENSILE PROPERTIES}

Tensile tests were conducted in air at room temperature on 0.25-in.diameter by 0.9-in.-long cylinders of $\mathrm{H}-327$ and $\mathrm{H}-451$ graphite irradiated in capsule OG-1. A crosshead speed of $0.005 \mathrm{in.} / \mathrm{mm}$ was used, and strains were measured with a 0.5-in. gauge length clip-on extensometer. The samples were cemented to metal end-pieces with high-strength epoxy cement, and the load was applied through roller-link chains to maintain uniaxial alignment during testing. Typical stress-strain curves for unirradiated and irradiated H-451 graphite are shown in Fig. 13. Because of the inelastic component in the deformation of graphite, the unloading curve does not retrace the initial loading curve but reaches zero load with a positive "permanent set." The second loading curve superimposes on the unloading curve, and beyond the first strain reversal point it forms a continuation of the initial loading curve. Irradiation greatly reduces the inelastic strain component and the stress-strain curve loses much of its curvature, particularly for irradiation at lower temperature (see Fig. 13). In the present tests, Young's modulus was measured from the 100to 1000-psi reloading portion of the curve, because this represents the effective modulus of a graphite component after its first stress excursion and because its slope is more accurately measured than that of a tangent to the curve at the origin.

The results of the tensile tests are listed in Tables 14 and 15 . The tensile data for unirradiated material were obtained from companion samples from the same log locations as the irradiated samples. The changes in strength and Young's modulus with neutron exposure are illustrated in Figs. 14 and 15. The strength and Young's modulus of H-327 graphite and H-451 graphite increased, with a greater increase occurring for lower-temperature irradiation than for higher-temperature 

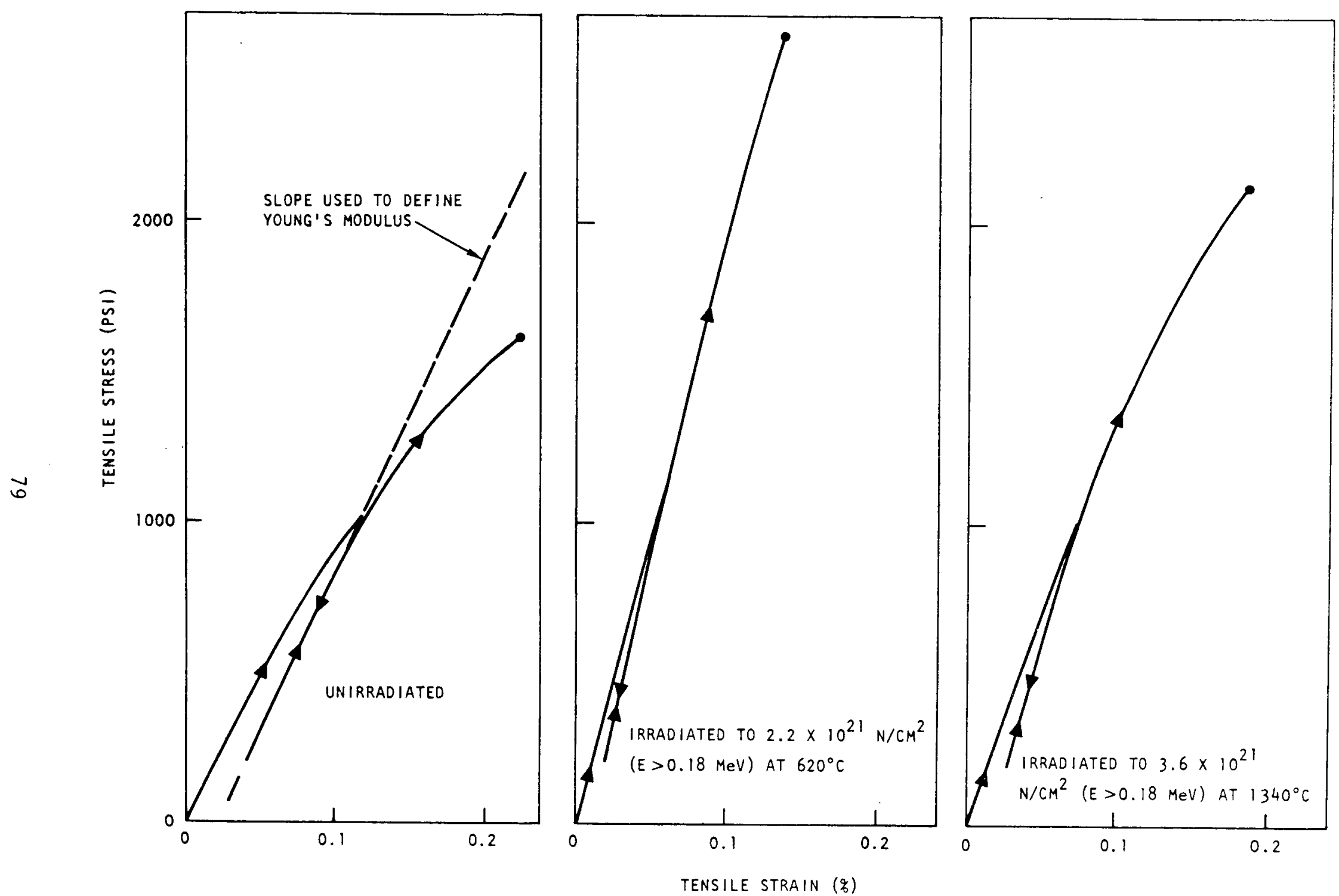

Fig. 13. Typical stress-strain curves of unirradiated and irradiated H-451 graphite 
TABLE 14

TENSILE TESTS ON H-327 GRAPHITE IRRADIATED IN OG- 1 CAPSULE

\begin{tabular}{|c|c|c|c|c|c|c|c|c|c|}
\hline $\begin{array}{c}\text { Cruclble } \\
\text { No. }\end{array}$ & $\begin{array}{l}\text { Hole } \\
\text { No. }\end{array}$ & $\begin{array}{l}\text { Irradiation } \\
\text { Temperature } \\
\left({ }^{\circ} \mathrm{C}\right)\end{array}$ & $\begin{array}{c}\text { Fluence } \times 10^{-21} \\
\left(\mathrm{n} / \mathrm{cm}^{2}\right) \\
(E>0.18 \mathrm{MeV})\end{array}$ & $\begin{array}{l}\log \\
\text { Location and } \\
\text { Orlentation }(a)\end{array}$ & $\begin{array}{c}\text { Spec Imen } \\
\text { No. }\end{array}$ & $\begin{array}{c}\text { Strain at } \\
\text { Fallure } \\
(\%)\end{array}$ & $\begin{array}{l}\text { Uitimate } \\
\text { Tensile } \\
\text { Strength } \\
\text { (psi) }\end{array}$ & $\begin{array}{l}\text { Young 's } \\
\text { Modylus }(b) \\
\left(10^{6} \text { pE } 1\right)\end{array}$ & $\begin{array}{l}\text { Secant } \\
\text { Modylus (c) } \\
\left(10^{6} \text { psi) }\right.\end{array}$ \\
\hline \multirow[t]{3}{*}{--} & \multirow[t]{3}{*}{--} & \multirow[t]{3}{*}{-} & \multirow[t]{3}{*}{0} & \multirow[t]{3}{*}{$\begin{array}{l}\text { E para } \\
\text { E para } \\
\text { E para } \\
\text { E para } \\
\text { E para } \\
\text { E para } \\
\text { E para } \\
\text { E para } \\
\text { E para } \\
\text { E para }\end{array}$} & $\begin{array}{r}1 \\
2 \\
3 \\
4 \\
5 \\
6 \\
7 \\
8 \\
9 \\
10\end{array}$ & $\begin{array}{l}0.226 \\
0.248 \\
0.170 \\
0.132 \\
0.250 \\
0.203 \\
0.176 \\
0.224 \\
0.180 \\
0.185 \\
\end{array}$ & $\begin{array}{l}1874 \\
2669 \\
1956 \\
1690 \\
2648 \\
2220 \\
2281 \\
2241 \\
2159 \\
2119 \\
\end{array}$ & $\begin{array}{l}1.61 \\
1.91 \\
2.16 \\
1.77 \\
1.87 \\
1.73 \\
2.49 \\
1.78 \\
1.85 \\
2.12 \\
\end{array}$ & $\begin{array}{l}0.83 \\
1.08 \\
1.15 \\
1.28 \\
1.06 \\
1.09 \\
1.30 \\
1.00 \\
1.20 \\
1.15 \\
\end{array}$ \\
\hline & & & & & Mean & 0.199 & 2186 & 1.93 & 1.11 \\
\hline & & & & & Std dev & \pm 0.035 & \pm 293 & \pm 0.25 & \pm 0.13 \\
\hline \multirow[t]{3}{*}{--} & \multirow[t]{3}{*}{--} & \multirow[t]{3}{*}{-} & \multirow[t]{3}{*}{$\dot{0}$} & \multirow[t]{3}{*}{$\begin{array}{l}\text { C para } \\
\text { C para } \\
\text { C para } \\
\text { C para } \\
\text { C para } \\
\text { C para } \\
\text { C para } \\
\text { C para }\end{array}$} & $\begin{array}{l}11 \\
12 \\
13 \\
15 \\
16 \\
17 \\
18 \\
19\end{array}$ & $\begin{array}{l}0.112 \\
0.147 \\
0.124 \\
0.160 \\
0.176 \\
0.105 \\
0.134 \\
-- \\
\end{array}$ & $\begin{array}{l}1141 \\
1508 \\
1182 \\
1324 \\
1569 \\
1222 \\
1406 \\
1222 \\
\end{array}$ & $\begin{array}{l}1.15 \\
1.43 \\
1.32 \\
1.12 \\
1.22 \\
1.43 \\
1.41 \\
1.33 \\
\end{array}$ & $\begin{array}{l}1.02 \\
1.03 \\
0.95 \\
0.83 \\
0.89 \\
1.16 \\
1.05 \\
-- \\
\end{array}$ \\
\hline & & & & & Mean & 0.137 & 1321 & 1.30 & 0.99 \\
\hline & & & & & Std dev & \pm 0.026 & \pm 158 & \pm 0.12 & \pm 0.11 \\
\hline \multirow[t]{3}{*}{--} & \multirow[t]{3}{*}{--} & \multirow[t]{3}{*}{--} & \multirow[t]{3}{*}{0} & \multirow[t]{3}{*}{$\begin{array}{l}C \text { perp } \\
C \text { perp } \\
C \text { perp } \\
C \text { perp } \\
C \text { perp } \\
C \text { perp } \\
C \text { perp } \\
C \text { perp } \\
C \text { perp } \\
C \text { perp } \\
C \text { perp } \\
C \text { perp } \\
C \text { perp }\end{array}$} & $\begin{array}{l}21 \\
22 \\
24 \\
25 \\
26 \\
28 \\
29 \\
30 \\
31 \\
32 \\
33 \\
34 \\
35\end{array}$ & $\begin{array}{c}0.144 \\
0.216 \\
0.256 \\
0.160 \\
0.230 \\
0.249 \\
-- \\
-- \\
-- \\
0.262 \\
0.240 \\
0.196 \\
0.336 \\
\end{array}$ & $\begin{array}{r}815 \\
896 \\
1161 \\
896 \\
957 \\
713 \\
244 \\
488 \\
468 \\
957 \\
1120 \\
754 \\
1201 \\
\end{array}$ & $\begin{array}{l}0.73 \\
0.67 \\
0.63 \\
0.77 \\
0.70 \\
0.51 \\
-- \\
-- \\
-- \\
0.58 \\
0.56 \\
0.62 \\
0.64 \\
\end{array}$ & $\begin{array}{l}0.57 \\
0.42 \\
0.45 \\
0.56 \\
0.42 \\
0.29 \\
-- \\
-- \\
-- \\
0.37 \\
0.47 \\
0.38 \\
0.36 \\
\end{array}$ \\
\hline & & & & & Mean & 0.229 & 820 & 0.64 & 0.43 \\
\hline & & & & & Std dev & \pm 0.055 & \pm 286 & \pm 0.08 & \pm 0.09 \\
\hline \multirow[t]{3}{*}{--} & \multirow[t]{3}{*}{--} & \multirow[t]{3}{*}{--} & \multirow[t]{3}{*}{$\begin{array}{l}0 \\
1\end{array}$} & \multirow[t]{3}{*}{$\begin{array}{l}\text { E perp } \\
\text { E perp } \\
\text { E perp } \\
\text { E perp } \\
\text { E perp } \\
\text { E perp } \\
\text { E perp } \\
\text { E perp } \\
\text { E perp } \\
\text { E perp } \\
\text { E perp } \\
\text { E perp } \\
\text { E perp } \\
\text { E perp } \\
\text { E perp } \\
\text { E perp } \\
\text { E perp } \\
\text { E perp } \\
\text { E perp }\end{array}$} & $\begin{array}{l}36 \\
37 \\
38 \\
39 \\
40 \\
41 \\
42 \\
43 \\
44 \\
45 \\
46 \\
47 \\
48 \\
49 \\
50 \\
51 \\
52 \\
53 \\
54\end{array}$ & $\begin{array}{l}0.368 \\
0.360 \\
0.373 \\
0.421 \\
0.373 \\
0.360 \\
0.332 \\
0.472 \\
0.405 \\
0.368 \\
0.338 \\
0.223 \\
0.305 \\
0.317 \\
0.392 \\
0.260 \\
0.372 \\
0.386 \\
0.232 \\
\end{array}$ & $\begin{array}{l}1609 \\
1426 \\
1548 \\
1711 \\
1507 \\
1385 \\
1385 \\
1691 \\
1589 \\
1528 \\
1324 \\
1019 \\
1365 \\
1182 \\
1487 \\
1263 \\
1405 \\
1691 \\
1059 \\
\end{array}$ & $\begin{array}{l}0.62 \\
0.68 \\
0.68 \\
0.68 \\
0.66 \\
0.62 \\
0.59 \\
0.56 \\
0.60 \\
0.69 \\
0.64 \\
0.66 \\
0.71 \\
0.59 \\
0.69 \\
0.74 \\
0.66 \\
0.71 \\
0.63 \\
\end{array}$ & $\begin{array}{l}0.44 \\
0.40 \\
0.42 \\
0.41 \\
0.40 \\
0.38 \\
0.42 \\
0.36 \\
0.39 \\
0.42 \\
0.39 \\
0.46 \\
0.45 \\
0.37 \\
0.38 \\
0.49 \\
0.38 \\
0.44 \\
0.46 \\
\end{array}$ \\
\hline & & & & & Mean & 0.350 & 1430 & 0.65 & 0.41 \\
\hline & & & & & Std dev & \pm 0.062 & \pm 200 & \pm 0.05 & 0.04 \\
\hline
\end{tabular}


TABLE 14 (Continued)

\begin{tabular}{|c|c|c|c|c|c|c|c|c|c|}
\hline $\begin{array}{c}\text { Cructhle } \\
\text { No. }\end{array}$ & $\begin{array}{l}\text { Hole } \\
\text { No. }\end{array}$ & $\begin{array}{c}\text { Irradiation } \\
\text { Temperature } \\
\left({ }^{\circ} \mathrm{C}\right)\end{array}$ & $\begin{array}{c}\text { Fluence } \times 10^{-21} \\
\left(\mathrm{n} / \mathrm{cm}^{2}\right) \\
(E>0.18 \mathrm{MeV})\end{array}$ & $\begin{array}{c}\log \\
\text { Location and } \\
\text { Orlentation }\end{array}$ & $\begin{array}{c}\text { Specimen } \\
\text { No. }\end{array}$ & $\begin{array}{l}\text { Strain at } \\
\text { Failure } \\
(\%)\end{array}$ & $\begin{array}{l}\text { U1timate } \\
\text { Tens1le } \\
\text { Strength } \\
\text { (psi) }\end{array}$ & $\begin{array}{l}\text { Young's } \\
\text { Modulus } \\
\left(10^{6} \text { ps } 1\right)\end{array}$ & $\begin{array}{l}\text { Secant } \\
\text { Mody1us (c) } \\
\left(10^{6} \text { psi) }\right.\end{array}$ \\
\hline \multirow[t]{2}{*}{2} & \multirow[t]{2}{*}{$\begin{array}{l}16 \\
19 \\
22 \\
25 \\
32 \\
35 \\
39 \\
42 \\
45 \\
49\end{array}$} & \multirow[t]{2}{*}{$\stackrel{700}{1}$} & \multirow[t]{2}{*}{2.9} & \multirow[t]{2}{*}{$\begin{array}{l}\text { E para } \\
\text { E para } \\
\text { E para } \\
\text { E para } \\
\text { E para } \\
\text { E para } \\
\text { E para } \\
\text { E para } \\
\text { E para } \\
\text { E para }\end{array}$} & $\begin{array}{l}351 \\
352 \\
353 \\
354 \\
355 \\
356 \\
357 \\
358 \\
359 \\
360\end{array}$ & $\begin{array}{l}0.109 \\
0.115 \\
0.089 \\
0.118 \\
0.093 \\
0.1114 \\
0.1112 \\
0.124 \\
0.091 \\
0.104 \\
\end{array}$ & $\begin{array}{l}2933 \\
3504 \\
2709 \\
3606 \\
2995 \\
3056 \\
3422 \\
3321 \\
3035 \\
2934 \\
\end{array}$ & $\begin{array}{l}3.93 \\
4.18 \\
3.77 \\
3.77 \\
4.38 \\
3.29 \\
3.85 \\
3.62 \\
4.23 \\
3.46 \\
\end{array}$ & $\begin{array}{l}2.69 \\
3.05 \\
3.04 \\
3.06 \\
3.22 \\
2.68 \\
3.06 \\
2.68 \\
3.34 \\
2.82 \\
\end{array}$ \\
\hline & & & & & $\begin{array}{c}\text { Mean } \\
\text { Std dev }\end{array}$ & $\begin{array}{r}0.107 \\
\pm 0.012\end{array}$ & $\begin{array}{l}3152 \\
\pm 278\end{array}$ & $\begin{array}{r}3.85 \\
\pm 0.33\end{array}$ & $\begin{array}{r}2.96 \\
\pm 0.20\end{array}$ \\
\hline \multirow[t]{3}{*}{4} & \multirow[t]{3}{*}{$\begin{array}{l}18 \\
22 \\
27 \\
31 \\
34 \\
37 \\
41 \\
44\end{array}$} & \multirow[t]{3}{*}{$\begin{array}{c}1100 \\
1100 \\
1050 \\
1 .\end{array}$} & \multirow[t]{3}{*}{$i^{3.7}$} & \multirow[t]{3}{*}{$\begin{array}{l}\text { E para } \\
\text { E para } \\
\text { E para } \\
\text { E para } \\
\text { E para } \\
\text { E para } \\
\text { E para } \\
\text { E para }\end{array}$} & $\begin{array}{l}361 \\
362 \\
363 \\
364(\mathrm{~d}) \\
365 \\
366 \\
367 \\
368\end{array}$ & $\begin{array}{c}0.095 \\
0.130 \\
0.094 \\
(0.057) \\
0.131 \\
0.102 \\
0.118 \\
0.115 \\
\end{array}$ & $\begin{array}{r}2485 \\
3198 \\
2445 \\
(1263) \\
3035 \\
2832 \\
2893 \\
2872 \\
\end{array}$ & $\begin{array}{r}3.32 \\
3.18 \\
3.22 \\
(2.67) \\
2.93 \\
3.14 \\
3.18 \\
3.61 \\
\end{array}$ & $\begin{array}{c}2.62 \\
2.46 \\
2.60 \\
(2.22) \\
2.32 \\
2.78 \\
2.45 \\
2.50 \\
\end{array}$ \\
\hline & & & & & Mean & 0.112 & 2822 & 3.23 & 2.53 \\
\hline & & & & & Std dev & \pm 0.014 & \pm 254 & \pm 0.19 & \pm 0.14 \\
\hline \multirow[t]{3}{*}{6} & \multirow[t]{3}{*}{$\begin{array}{l}16 \\
19 \\
22 \\
25 \\
32 \\
35 \\
39 \\
42 \\
45 \\
49\end{array}$} & \multirow[t]{3}{*}{1020} & \multirow[t]{3}{*}{$i^{3.4}$} & \multirow[t]{3}{*}{$\begin{array}{l}C \text { perp } \\
C \text { perp } \\
C \text { perp } \\
C \text { perp } \\
C \text { perp } \\
C \text { perp } \\
C \text { perp } \\
C \text { perp } \\
C \text { perp } \\
C \text { perp }\end{array}$} & $\begin{array}{l}401 \\
402 \\
403 \\
404 \\
405 \\
406 \\
407 \\
408 \\
409 \\
410\end{array}$ & $\begin{array}{l}0.154 \\
0.154 \\
0.097 \\
0.126 \\
0.162 \\
0.111 \\
0.058 \\
0.110 \\
0.062 \\
0.096 \\
\end{array}$ & $\begin{array}{r}1080 \\
1120 \\
856 \\
733 \\
1243 \\
978 \\
774 \\
1283 \\
754 \\
795 \\
\end{array}$ & $\begin{array}{l}1.18 \\
0.96 \\
1.22 \\
0.94 \\
1.08 \\
1.33 \\
1.66 \\
1.44 \\
1.30 \\
1.20 \\
\end{array}$ & $\begin{array}{l}0.86 \\
0.73 \\
0.88 \\
0.58 \\
0.77 \\
0.88 \\
1.33 \\
1.17 \\
1.22 \\
0.83 \\
\end{array}$ \\
\hline & & & & & Mean & 0.110 & 962 & 1.23 & 0.93 \\
\hline & & & & & Std dev & \pm 0.032 & \pm 198 & \pm 0.20 & \pm 0.23 \\
\hline \multirow[t]{3}{*}{8} & \multirow[t]{3}{*}{$\begin{array}{l}18 \\
18 \\
21 \\
21 \\
24 \\
24 \\
30 \\
30 \\
33 \\
33\end{array}$} & \multirow[t]{3}{*}{900} & \multirow[t]{3}{*}{$2 j^{6}$} & \multirow[t]{3}{*}{$\begin{array}{l}\text { C para } \\
\text { C para } \\
C \text { para } \\
\text { C para } \\
\text { C para } \\
\text { C para } \\
\text { C para } \\
C \text { para } \\
\text { C para } \\
C \text { para }\end{array}$} & $\begin{array}{l}279 \\
280 \\
281 \\
282 \\
283 \\
284 \\
285 \\
286 \\
287 \\
288\end{array}$ & $\begin{array}{c}0.075 \\
0.099 \\
0.113 \\
0.084 \\
0.092 \\
0.108 \\
-- \\
0.082 \\
0.142 \\
0.106 \\
\end{array}$ & $\begin{array}{l}1996 \\
2098 \\
2648 \\
2058 \\
2017 \\
2282 \\
1732 \\
1752 \\
2159 \\
2526 \\
\end{array}$ & $\begin{array}{l}3.12 \\
2.85 \\
3.38 \\
2.83 \\
2.67 \\
2.97 \\
3.12 \\
3.12 \\
2.59 \\
3.16 \\
\end{array}$ & $\begin{array}{l}2.66 \\
2.12 \\
2.34 \\
2.45 \\
2.19 \\
2.11 \\
-. . \\
2.14 \\
1.52 \\
2.38\end{array}$ \\
\hline & & & & & Mean & 0.100 & 2127 & 3.02 & 2.21 \\
\hline & & & & & Std dev & \pm 0.019 & \pm 281 & \pm 0.27 & \pm 0.30 \\
\hline \multirow[t]{3}{*}{9} & \multirow[t]{3}{*}{$\begin{array}{l}16 \\
19 \\
22 \\
25 \\
32 \\
35 \\
39 \\
42 \\
45 \\
49\end{array}$} & \multirow[t]{3}{*}{810} & \multirow[t]{3}{*}{2.1} & \multirow[t]{3}{*}{$\begin{array}{l}\text { E para } \\
\text { E para } \\
\text { E para } \\
\text { E para } \\
\text { E para } \\
\text { E para } \\
\text { E para } \\
\text { E para } \\
\text { E para } \\
\text { E para }\end{array}$} & $\begin{array}{l}369 \\
370 \\
371 \\
372 \\
373 \\
374 \\
375 \\
376 \\
377 \\
378\end{array}$ & $\begin{array}{l}0.143 \\
0.110 \\
0.099 \\
0.151 \\
0.107 \\
0.112 \\
0.118 \\
0.110 \\
0.129 \\
0.100 \\
\end{array}$ & $\begin{array}{l}3259 \\
2730 \\
2628 \\
2995 \\
2546 \\
2832 \\
3015 \\
2445 \\
2913 \\
2506 \\
\end{array}$ & $\begin{array}{l}2.91 \\
3.10 \\
3.57 \\
2.55 \\
3.18 \\
3.26 \\
3.10 \\
3.04 \\
2.99 \\
3.34 \\
\end{array}$ & $\begin{array}{l}2.28 \\
2.48 \\
2.65 \\
1.98 \\
2.38 \\
2.53 \\
2.56 \\
2.22 \\
2.26 \\
2.51\end{array}$ \\
\hline & & & & & Yean & 0.118 & 2787 & 3.10 & 2.39 \\
\hline & & & & & Std dev & \pm 0.017 & \pm 249 & \pm 0.26 & \pm 0.19 \\
\hline
\end{tabular}

(a) $E$ = edge, $C$ - center; para - parallel, perp = perpendicular.

(b) 100-1000 psi (parallel samples) or 100-600 psi (perpendicular samples) after preloading to 1000 pei (patallel samples) or 600 psi (perpendicular samples), unloading sample, and reloading.

(c) litimate tensile strength divided by fracture strain.

(d) Sample contalned flaw; excluded from averages. 
TENSILE TESTS ON H-451 GRAPHITE IRRADIATED IN OG- 1 CAPSULE

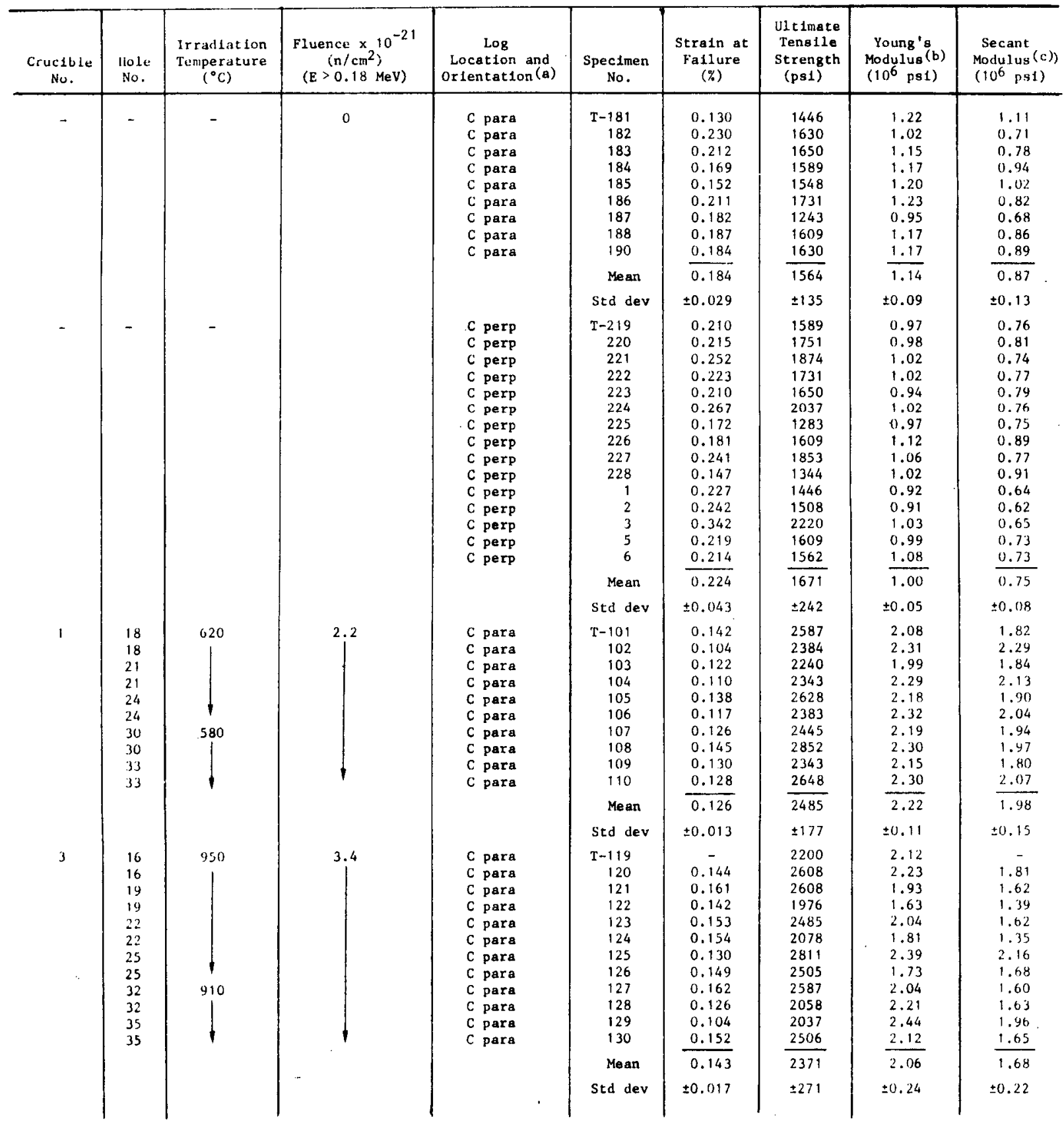


TABLE 15 (Continued)

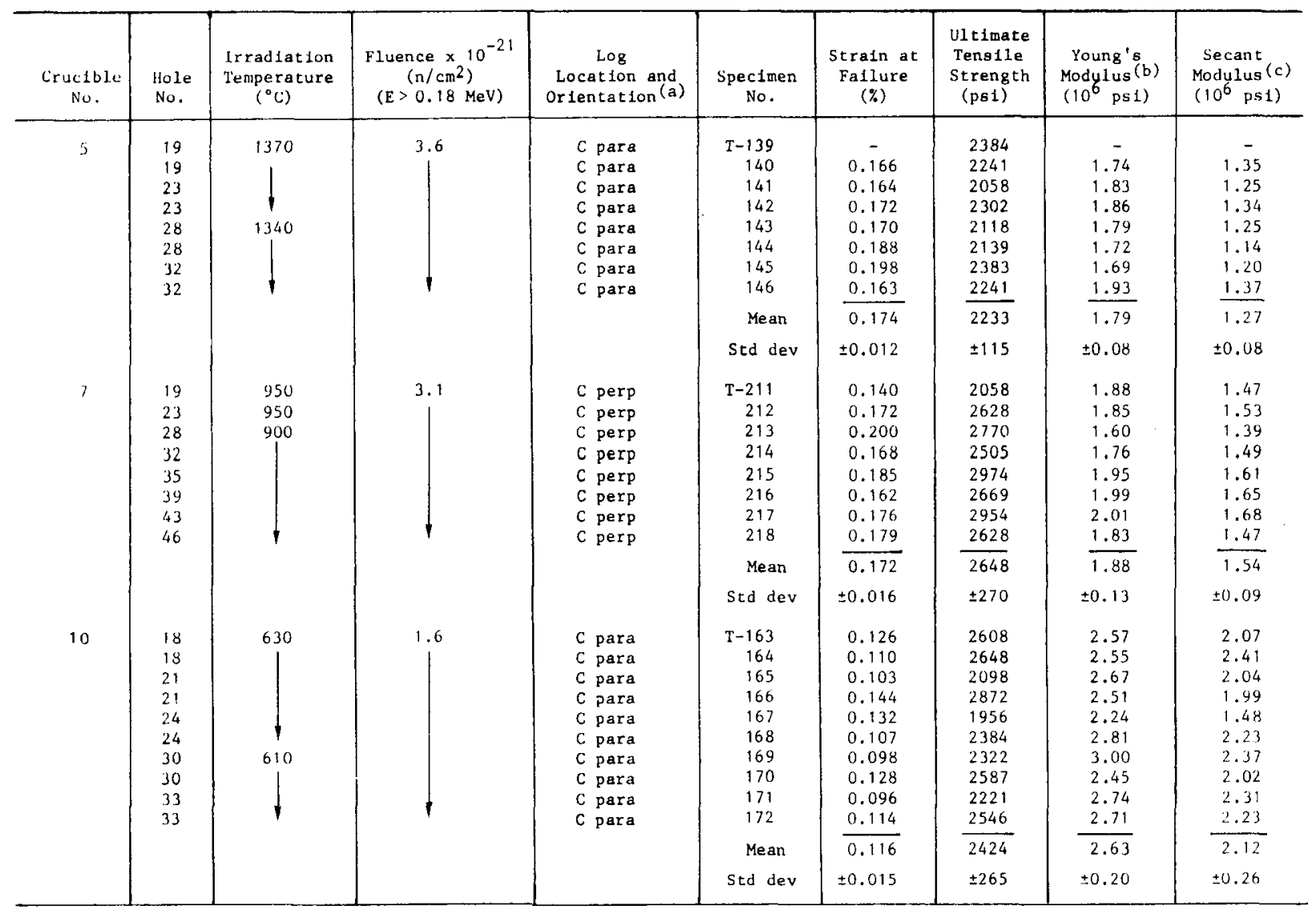

${ }^{(a)} C$ a center, $E=$ edge; para $=$ parallel, perp - perpendicular.

(b) 100-1000 psi, after preloading to 1000 psi, unloading sample, and reloading.

(c) Ultimate tensile strength divided by fracture stratn. 

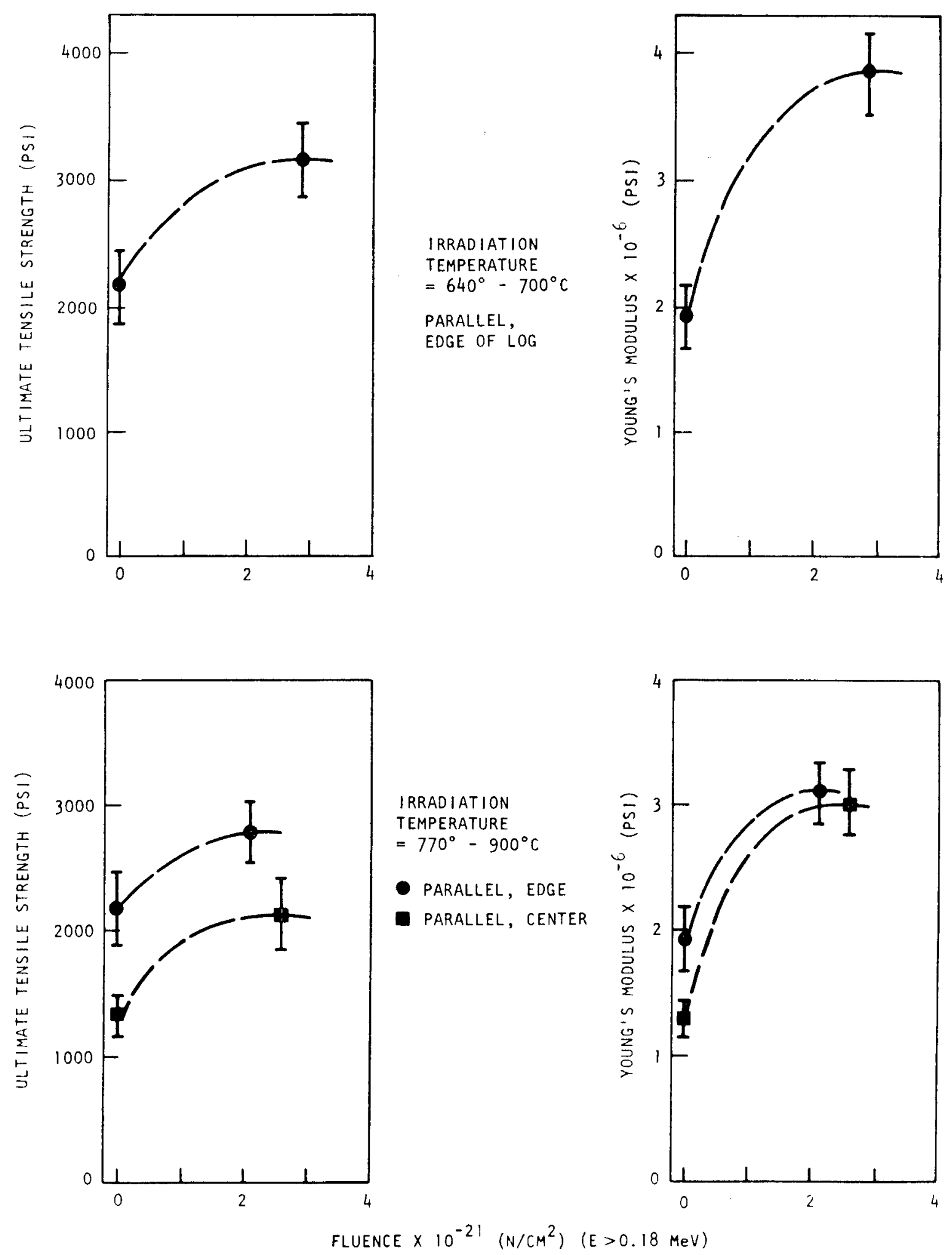

Fig. 14a. Ultimate tensile strength and Young's modulus of H-327 graphite irradiated at $640^{\circ}$ to $900^{\circ} \mathrm{C}$ as a function of fast neutron fluence. Error bands indicate \pm one standard deviation. 


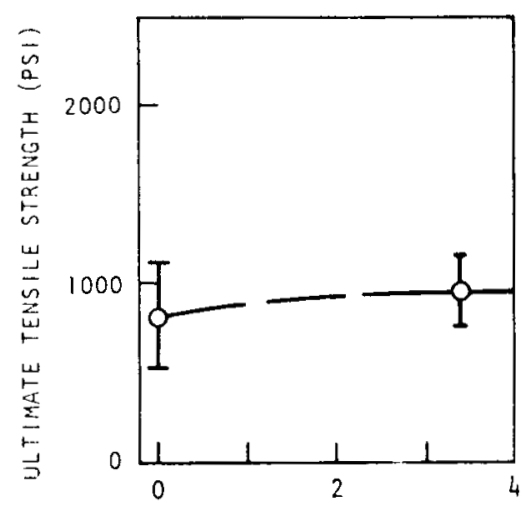

IRRADIATION

TEMPERATURE

$=960^{\circ}-1020^{\circ} \mathrm{C}$

PERPENDICULAR,

CENTER OF LOG
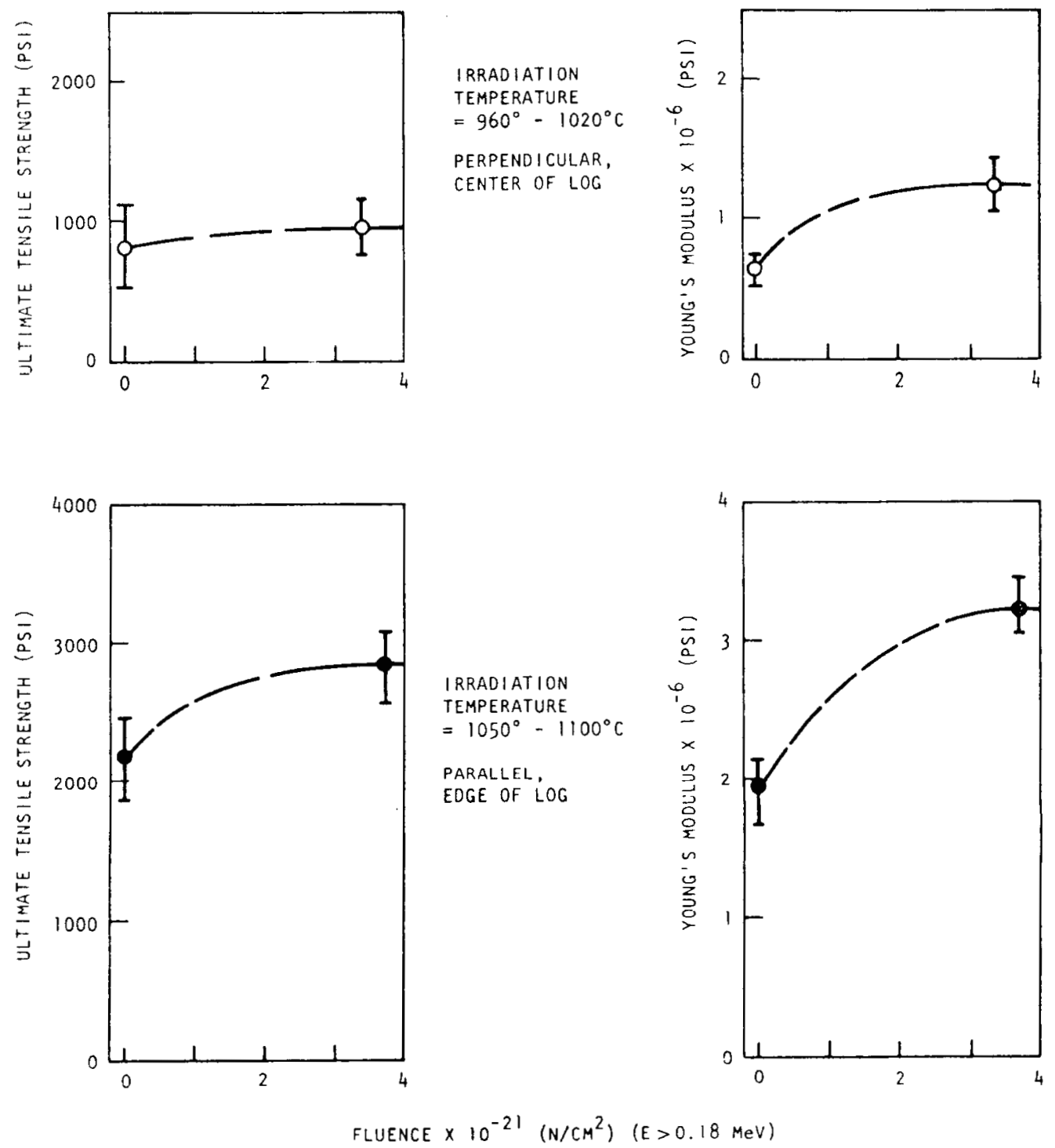

Fig. 14b. Ultimate tensile strength and Young's modulus of $\mathrm{H}-327$ graphite irradiated at $960^{\circ}$ to $1100^{\circ} \mathrm{C}$ as a function of fast neutron fluence. Error bands indicate \pm one standard deviation. 

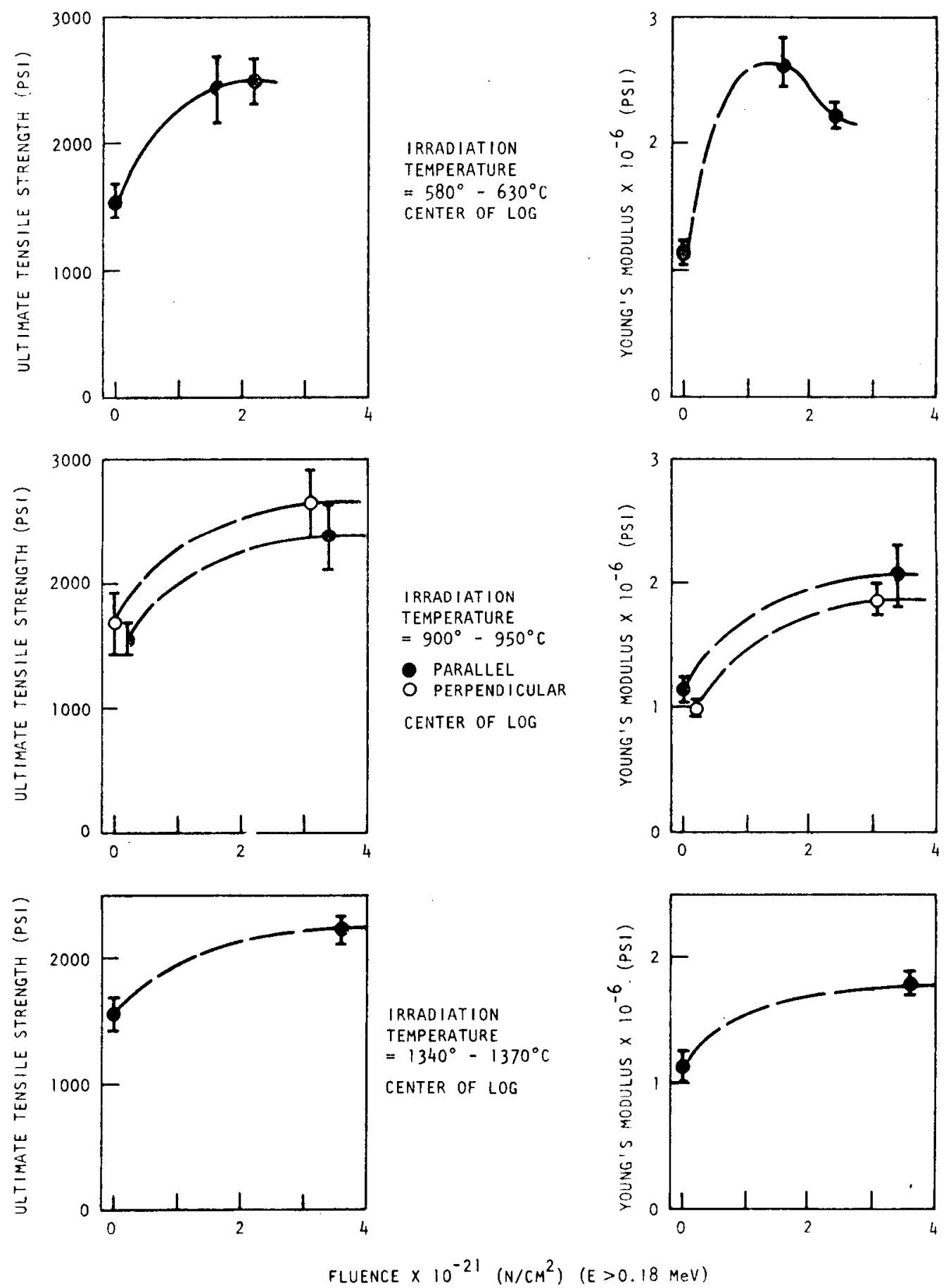

Fig. 15. Ultimate tensile strength and Young's modulus of H-451 graphite as a function of fast neutron fluence. Error bands indicate \pm one standard deviation. 
irradiation. The percentage increases in strength were in the range $30 \%$ to $60 \%$, while increases in elastic modulus ranged from $60 \%$ to $130 \%$. There was no significant change in the scatter of the strength data following irradiation, with the coefficient of variation remaining at about $10 \%$. Average strength values (plus or minus one standard deviation) for material from the center of the log (the weakest portion) irradiated to 2.6 to $3.4 \times 10^{21} \mathrm{n} / \mathrm{cm}^{2}$ at $860^{\circ}$ to $1080^{\circ} \mathrm{C}$ were as follows:
H-327 paralle1
$2127 \pm 281$ psi
$\mathrm{H}-327$ perpendicular
$962 \pm 198$ psi
H-451 parallel
$2371 \pm 271$ psi
H-451 perpendicular
$2648 \pm 270$ psi 


\section{IMPACT OF TEST DATA ON CURRENT DESIGN-BASIS CURVES}

\subsection{DIMENSIONAL CHANGES}

The dimensional changes observed in capsule OG-1 for $\mathrm{H}-451$ and $\mathrm{H}-429$ graphites are the first measurements on a full-size near-isotropic graphite produced for use in the HTGR. The data agreed very well in the perpendicular direction with the predicted curves used in design. The parallel-direction data exhibited $\sim 20 \%$ less contraction than predicted. The OG-1 data have been fit, and new dimensional change curves have been generated (Figs. 4 and 5).

The OG-1 data on $\mathrm{H}-327$ implied that $\mathrm{H}-327$ was more dimensionally stable than earlier data had indicated. These data will be combined with earlier data to obtain a new dimensional change curve representative of $\mathrm{H}-327$.

\subsection{THERMAL EXPANSIVITY}

The measured thermal expansivities of irradiated $\mathrm{H}-451$ and $\mathrm{H}-429$ graphites showed little change from unirradiated values. The present design curves overestimate the change in thermal expansivity by $10 \%$ to $30 \%$. The curves will be changed in light of the OG-1 data.

The design-basis curves for the thermal expansivity of $\mathrm{H}-327$ predict no change under irradiation to $8 \times 10^{21} \mathrm{n} / \mathrm{cm}^{2}$. The $0 \mathrm{G}-1$ data indicate a small increase in the coefficient of thermal expansion in the parallel direction for $\mathrm{H}-327$. This small increase is not significant in light of the decreasing importance of $\mathrm{H}-327$ to large HTGR design. 


\subsection{THERMAL CONDUCTIVITY}

The measured thermal conductivities of irradiated H-451 graphite were within a few percent of the predicted curves in Ref. 4. However, the conductivities of the H-327 samples irradiated at $600^{\circ}$ and $900^{\circ} \mathrm{C}$ averaged $25 \%$ lower than the predicted curves for both the parallel and perpendicular directions (Ref. 4). The samples irradiated at $1350^{\circ} \mathrm{C}$ agreed with the predicted curves. Because of the decline in importance of needle-coke graphites to the HTGR program, there are no present plans to revise the design-basis thermal conductivity curves for H-327 graphite.

\subsection{MECHANICAL PROPERTIES}

The design-basis curves for change in modulus during irradiation are being revised to represent the fluence and temperature dependence observed in OG-1. The present design-basis curves, based on changes in the dynamic modulus of Gilsocarbon-based graphites, underestimate the change in modulus up to $65 \%$ at $600^{\circ} \mathrm{C}$, with the error decreasing to $5 \%$ at $1350^{\circ} \mathrm{C}$. The change in ultimate tensile strength with irradiation is also being tabulated for incorporation in a revised design basis. No increase in ultimate tensile strength with irradiation was previously assumed, so OG-1 data will justify the use of increased allowable stress limits. However, the higher absolute limits will probably result in nearly the same safety margins owing to the increased effects on the modulus. 


\section{CONCLUSIONS}

The petroleum-coke near-isotropic graphites (H-451, H-429, H-453) exhibit significantly improved dimensional stability over other productionscale nuclear graphites ( $H-327, \mathrm{CHN}, \mathrm{P}_{3} \mathrm{JHAN}$ ). The petroleum-coke nearisotropic graphites approach the dimensional stability of the Gilsocoke graphites. The production-grade needle-coke graphite $\mathrm{H}-327$ displayed better dimensional stability than in earlier tests, possibly because of improvements during production and/or improvements in capsule instrumentation and analysis. The pitch-coke-based $\mathrm{P}_{3}$ JHAN graphite showed poorer dimensional stability than either $\mathrm{H}-451$ or $\mathrm{H}-327$ graphites under the irradiation conditions of capsule OG-1.

Irradiation-induced changes in the thermal expansivity of $\mathrm{H}-327$ graphite are insignificant. Irradiation causes a small increase in the thermal expansivity of $\mathrm{H}-429$ and $\mathrm{H}-451$ graphites below about $900^{\circ} \mathrm{C}$ and a slight decrease at higher temperatures.

Irradiation reduces both the thermal conductivity and the temperature dependence of the thermal conductivity of $\mathrm{H}-327$ and $\mathrm{H}-451$ graphites. After irradiation at $600^{\circ}$ and $900^{\circ} \mathrm{C}$, the radial conductivity of $\mathrm{H}-451$ graphite is about $50 \%$ higher than the radial conductivity of irradiated H-327 graphite and about equal to the axial conductivity of $\mathrm{H}-327$ graphite.

Irradiation increases both the strength and Young's modulus of $\mathrm{H}-327$ and H-451 graphites, with a greater increase occurring at lower irradiation temperatures. Percentage increases in strength fall in the range $30 \%$ to $60 \%$, while increases in Young's modulus range from $60 \%$ to $130 \%$ for irradiation temperatures of $600^{\circ}$ to $1350^{\circ} \mathrm{C}$. 


\section{ACKNOWLEDGMENTS}

Design, construction, and operational monitoring of capsule OG-1 was performed by the Capsule Irradiation Branch under the supervision of J. S. Steibel and J. M. McNair. Other work was conducted by L. Bailey, G. B. Engle, H. H. Evans, W. R. Johnson, C. H. Richards, and J. R. Whaley. Thanks are extended to all these individuals. 


\section{REFERENCES}

1. "Fabrication and Assemb1y Report for the OG-1 Irradiation Capsule, GA Capsule Irradiation Branch Document No. OG-1-1," unpublished data.

2. "Final Test Operation Report - OG-1, GA Capsule Irradiation Branch Document No. OG-1-2," unpublished data.

3. Beavan, L. A., General Atomic Company, "Materials Inventory, OG-1 Irradiation Capsule," unpublished data.

4. Price, R. J., "Review of the Thermal Conductivity of Nuclear Graphite Under HTGR Conditions," Gulf General Atomic Report Gulf-GA-A12615, September 7, 1973. 